\title{
Fluoxetine and motor imagination to facilitate recovery after ischemic stroke
}

Hanneke I Berends - van Genderen 



\section{FLUOXETINE AND MOTOR IMAGINATION TO FACILITATE RECOVERY AFTER ISCHEMIC STROKE}

Hanneke I Berends - van Genderen 
Samenstelling promotiecommissie

Voorzitter/secretaris

Prof. dr. ir. J.W.M. Hilgenkamp

Promotoren

Prof. dr. ir. M.J.A.M. van Putten

Prof. dr. M.J. IJzerman

Co-promotor

Dr. K.L.L. Movig

Referent

Dr. J. Hofmeijer

Leden

Prof. dr. G. Kwakkel

Prof. dr. E.N. van Roon

Prof. dr. ir. H.J. Hermens

Prof. dr. J.S. Rietman

\section{Paranimfen}

Marit Dekker

Thijs Krabben

\section{Ontwerp omslag}

P. van Genderen
University of Twente

University of Twente

University of Twente

Medisch Spectrum Twente

University of Twente

VU University Medical Center

University of Groningen

University of Twente

University of Twente

\section{Drukwerk}

Ipskamp 


\title{
FLUOXETINE AND MOTOR IMAGINATION TO FACILITATE RECOVERY AFTER ISCHEMIC STROKE
}

\author{
PROEFSCHRIFT
}

ter verkrijging van

de graad van doctor aan de Universiteit Twente, op gezag van de rector magnificus, prof. dr. H. Brinksma, volgens besluit van het College voor Promoties in het openbaar te verdedigen op woensdag 9 maart 2016 om 14:45 uur

door

Hanneke Irene Berends - van Genderen

Geboren op 16 januari 1980

te Enschede 
Dit proefschrift is goedgekeurd door de promotoren:

Prof. dr. ir. M.J.A.M. van Putten

Prof. dr. M.J. IJzerman

en door de co-promotor:

Dr. K.L.L. Movig

ISBN: 9789036540445

DOI: $10.3990 / 1.9789026540445$

Copyright @2016 H.I. Berends - van Genderen, Arnhem, The Netherlands

All rights reserved. No part of this book may be reproduced, stored in a retrieval system, or transmitted, in any form or by any means, electronic, mechanical, photocopying, recording, or otherwise, without prior written permission of the author. 


\section{Table of contents}

$\begin{array}{lll}\text { Chapter } 1 \quad \text { General introduction } & 7\end{array}$

Chapter 2 The clinical use of drugs influencing neurotransmitters in the brain to promote motor recovery after stroke: a Cochrane systematic review

Chapter 3 Single dose of fluoxetine increases muscle activation in 45 chronic stroke patients

Chapter 4 Relation between the effects of a single dose of 59 fluoxetine on brain activity patterns, muscle activation and motor function in chronic stroke patients

Chapter 5 Differential cortical activation during observation and 75 observation-and-imagination

Chapter $6 \quad$ Influence of a single dose of fluoxetine on brain activity 95 patterns during imagination and execution of a movement in chronic stroke patients

Chapter $7 \quad$ General discussion and Future perspectives

Summary

Samenvatting

Dankwoord

Biografie 



\section{CHAPTER 1}

General Introduction 


\subsection{History of stroke}

More than 2400 years ago, Hippocrates, the father of medicine, first recognized stroke. He described it as "the sudden onset of paralysis", and called it "apoplexy", which means "struck down by violence" in Greek. At the time, physicians had little knowledge of the anatomy and function of the brain and the only therapy was to care for and to feed the patient.

Johann Jacob Wepfer (born in Switzerland, 1620) was the first to identify postmortem signs of bleeding in the brain of patients who died of apoplexy. He also discovered that blockage of one of the brain's blood vessels could cause apoplexy. Medical science continued to study the cause, symptoms, and treatment of apoplexy and only in the $20^{\text {th }}$ century the term apoplexy was changed into the terms "stroke" or "cerebral vascular accident". Nowadays, much research is done on the cause, risk factors, prevention, and treatment of stroke. However, stroke remains to be the leading cause of disability in many industrialized countries.

\subsection{The effects of a stroke}

Eighty-five percent of all strokes is ischemic, $15 \%$ of the patients suffer from hemorrhagic stroke. The effects depend on the type and size of stroke.

Stroke occurs when the blood supply of the brain is interrupted or reduced, leading to a lack of oxygen and nutrients. The infarcted area can be divided into regions of different degrees of damage. The neurons in the ischemic core are irreversibly damaged. The ischemic core is surrounded by a rim of moderate ischemic tissue, the "penumbra", with impaired neuronal function but preserved cellular metabolism and viability (Hakim 1987). In the penumbra (partial) recovery is possible, but only if reperfusion is established within hours or a few days, otherwise cell death still occurs. The eloquent area of the penumbra is a region with reduced blood supply (oligaemia), which is surrounded by healthier tissue (Fig 1).

The lack of oxygen and nutrients causes a cascade of devastating effects in the neurons. The failure of energy-dependent processes disturbs the balance between intracellular and extracellular concentrations of potassium and sodium, resulting in persistent elevated intracellular calcium. This causes cells in the ischemic core to die from apoptosis and necrosis, and occurs within minutes after the ischemic infarct. In addition, the membrane depolarization by the imbalanced ion concentrations causes a sudden high increase in glutamate concentrations, which results in activation of the N-Methyl-D-aspartate (NMDA) receptor and $\alpha$-amino-3hydroxy-5-methyl-4-isoxazolepropionic acid receptor (AMPA) receptor, leading to further membrane depolarization and (again) a greater calcium influx, exacerbating intracellular calcium overload. 
Oxidative stress is another major component of the ischemic stroke cascade. High levels of reactive oxygen species overwhelm antioxidant defenses, and cellular macromolecules are damaged, leading to apoptosis and necrosis (Doyle 2008).

In the penumbra, neuronal dysfunction is mainly caused by a failure of synaptic transmission and not by neuronal death. The degree of motor recovery after stroke depends largely on the recovery of neurons in this area. Long-lasting synaptic silence is thought to be a cause of persistent symptoms after mild to moderate cerebral ischemia (Symon 1977, Astrup 1977, Astrup 1981, Hofmeijer, 2012).

In the penumbra and in the cortical tissue of the intact brain, self-propagating waves of electrochemical activity may occur, as well: cortical spreading depressions (CSD). CSD causes a cellular depolarization, depressed neuroelectrical activity, and increased glutamate release and loss of membrane ionic gradients (Gonzalez, Leroux, 1992; Lauritzen, 2011; Zandt, 2013). Recurrent periinfarct depolarizations (PIDs which can be compared to CSD) propagate through the penumbra and are thought to increase the ischemic injury by accumulation of calcium within the neurons (Dreier 2011, Lauritzen 2011). In addition, cellular inflammatory response and the cytokine inflammatory response contribute to ischemic injury (Doyle 2008).

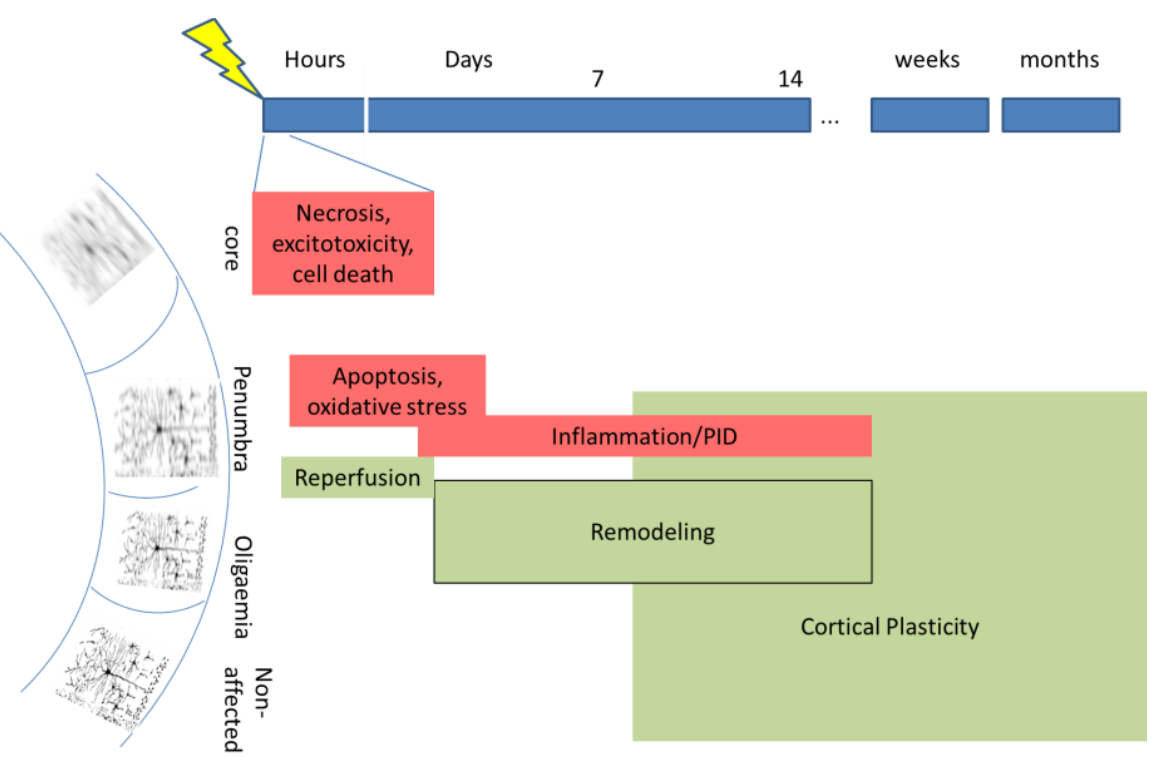

Figure 1 at the left side of the figure, a schematic presentation is given of the cortex after ischemic stroke. The red text boxes indicate the injury caused by stroke; the green text boxes indicate the mechanisms of recovery. The boxes are ordered by time (horizontal) and cortical area (vertical) (adapted from http://pt851.wikidot.com/stroke-cell-bio). 


\subsection{Motor recovery}

Our ability to learn or relearn tasks is based on the plasticity of our brain (Rossini 2003, Pascual-Leone 2005, Kleim 2008). Brain plasticity is the ability of the brain to change and adapt to a new situation, or new experiences. After stroke, redundant connections may take over the function of the destroyed ones. During motor recovery of highly impaired patients, the ipsilateral (non-affected) hemisphere may compensate for the loss of function at the lesioned side (Ward 2003, Biernaski 2005, Tangwiriyasakul 2014). This process can occur over days, weeks and even months after stroke. The taking over of function by redundant areas depends on the presence of genes and proteins that are important for neuronal growth, synaptogenesis and proliferations of dendritic spines. These genes and proteins are increased in the first weeks following stroke. For the rehabilitation it is therefore important to be initiated in time, as the largest plasticity can be expected in this time window (Murphy 2009).

Recovery of motor function after stroke can take place by "true recovery" or by behavioral compensation. True recovery can be achieved when destroyed circuits are replaced, or restored. In the first hours to few days after stroke, true recovery of function may result from restoration of damaged synapses between previously connected neurons in the penumbra (Hasbani 2000, Murphy 2009, Hofmeijer 2012).

Cortical plasticity is established by several mechanisms including unmasking of silent synapses, sprouting of nonaffected fibers, regeneration of axons and longterm potentiation (LTP) (Hasbani 2000, Chen 2002, Gu 2002, Carmichael 2003, Rossini 2004). An increased amount of neurotransmitters facilitates N-methyl-Daspartate (NMDA) receptor activation, which is thought to boost synaptic modifications, which are important to facilitate the mechanisms inducing cortical plasticity.

\subsection{Facilitating motor recovery after stroke}

In the acute phase after stroke, reperfusion of the penumbra can be accomplished by intravenous thrombolysis with recombinant tissue plasminogen activator (Morettia, 2015). In addition, the amount of harmful PIDs in the penumbra might be decreased using hypothermia (Karibe 1994, Erecinska 2003, Campos 2012, Yenary 2012).

Another way to limit the injury in the penumbra is by neuroprotection, antagonizing the harmful molecular and cellular events. This allows brain cells to survive the reduced blood flow and stabilize the penumbra (Morettib, 2015). The administration of neuroprotective agents to minimize the effects of the stroke is widely studied, for example by diminishing excitotoxicity by blocking the glutamate binding sites on NMDA and AMPA receptors, reducing the calcium influx. Promising results are found after administration of magnesium $\left(\mathrm{MgSO}_{4}\right)$ 
within 2 hours after stroke onset (Saver 2014). The effects of many other neuroprotective agents like intravenous albumin (Belayev 2001, DeFazio 2012, Chen 2013), minocycline (Fan 2013), edaravone (Lapchak 2010), ebselen (Imai 2003, Labchak 2003), have been widely studied. However, the results of all of these agents are not proven in human stroke (for an extensive review see Morettib, 2015).

After these treatments to reduce the effects of the stroke in the acute phase, the brain has to regain its functions by rehabilitative therapies. Physical therapies as well as neuropharmacological treatments are widely studied.

In the first period after stroke, environmental experience can markedly affect the properties and function of the adult brain. Sensory-motor and cognitive stimulation is found to promote regaining of function and can, for example, be achieved by social interaction, computer games and virtual reality (Laver 2015). Another possibility to enrich the rehabilitation is to stimulate the imagination, or observation of movements (Rizzolatti 2004, 2009).

Constraint-induced movement therapy is another therapeutic approach to improve stroke recovery. The enforced use of the impaired upper extremity is thought to promote functional recovery, by providing "learned non-use" of the affected side (Sawaki 2008).

\subsubsection{The impact of Observation and Imagination of movement}

The mechanisms inducing cortical plasticity require activity of neurons. Therefore, brain plasticity in the areas concerning movement occurs only when a movement is practiced, usually during physical exercise. Studies have shown that certain brain areas which are activated during the execution of a movement are also activated during observation or imitation of a movement (Cochin 1999, Babiloni 2002, Rizzolatti 2004, Calmels 2006, De Vries 2007, Rizzolatti 2009 Tangwiriyasakul 2014). This mirror mechanism plays a fundamental role in the understanding of action. The "mirror system" is thought to be involved in imitation as a largely overlapping circuit between passive observation of actions and observing to imitate. The findings that (1) observation of action recruits the motor system in the same way as does motor execution and (2) during the imitation of a novel motor pattern neurons in the motor areas are active from the observation phase until the execution of a new action, offer the possibility of improving motor performance through systematic exercise based on careful observation and imitation of everyday actions (Francescini 2010, Buccino 2014).

Ertelt et al studied the effects of observation of action on rehabilitation of motor deficits after stroke. They found "pieces of evidence suggesting that observation of action has a positive additional impact on recovery of motor functions after stroke by reactivation of those motor areas, that contain the action observation/action execution matching system" of the brain (Ertelt 2007). Page et al compared the 
efficacy of a rehabilitation program incorporating mental practice of specific arm movements with a placebo condition. They also found support for the efficacy of mental practice for rehabilitation of motor function in patients with chronic stroke (Page 2007).

\subsubsection{The effects of psychoactive drugs}

Besides physical therapy, pharmacological intervention might facilitate neuronal processes resulting in a promotion of recovery of motor function. The administration of amphetamine (Boyeson 1990, Walker-Batson 1995, Martinsson 2003), methylphenidate (Grade 1998, Tardy 2006), levodopa (Scheidtmann 2001, Acler 2009), and selective serotonin reuptake inhibitors (SSRIs) (Pariente 2001, Chollet 2011), are suggested to facilitate the neural processes inducing motor (re-) learning. The exact mechanism of amphetamine action is not known, but it is thought to be a modulator of cortical excitation, leading to facilitation of learning and motor skills (Barbay 2009). Methylphenidate administered to patients suffering from a subcortical stroke led to modulation of cerebral activation and plasticity, which was correlated with improvement in motor performance, by increasing the release of norepinephrine and dopamine (Tardy 2006, Weikop 2007). Levodopa increases the release of norepinephrine and is also involved in mediating actions through dopamine receptors in the primary motor cortex, which have been implicated in long-term plasticity (Ruscher 2012).

Fluoxetine is a selective serotonin reuptake inhibitor (SSRI) increasing serotonin concentration in the synaptic cleft between neurons in the brain. The administration of fluoxetine seems to be very promising in improving the recovery of motor function after stroke. It increases serotonin concentrations by inhibiting the reuptake of serotonin by the presynaptic terminal. Serotonin (5hydroxytryptamine, 5-HT) is a catecholaminergic neurotransmitter. The serotonergic pathways originate in the raphe nuclei and propagate to many structures of the brain (Figure 1). Projections are so extensive that virtually every neuron in the brain may be contacted by a serotonergic fiber. Besides the other projections to non-motor areas, serotonergic fibers project directly to $\alpha$-motor neurons and also innervate secondary motor structures. Serotonin is thought to facilitate motor output and simultaneously suppress sensory information processing. It is hypothesized that serotonergic neurons play an auxiliary role in coordinating appropriate autonomic and neuroendocrine outputs to the ongoing tonic or repetitive motor activity (Jacobs 1999).

After administration of a single dose of SSRI in healthy subjects changes were found in the activity of areas of the brain involved in motor function (Loubinoux 1999, Loubinoux 2002). These changes were found during active motor tasks. In addition, an increased excitability of the neurons in the motor cortex was found (Ilic 2002, Pleger 2004). The increased excitability and activity of areas in the brain 
involved in motor function might facilitate the plasticity of the brain and improve (re-)learning of movements.

\subsection{Neurophysiological techniques to evaluate stroke recovery}

\subsubsection{Electroencephalography (EEG)}

The EEG mainly results from synaptic activity of cortical pyramidal cells. Synchronous excitatory and inhibitory postsynaptic potentials of these neurons result in extracellular currents sufficiently large to allow voltage recordings from the scalp: the electroencephalogram. The EEG is characterized by various rhythms, typically evaluated in the frequency range $0.1-35 \mathrm{~Hz}$, but lower (infraslow activity) and higher frequencies (e.g. high gamma oscillations) exist as well (Holmes 2007, Van Putten 2009). Physiological rhythms may show various modulations if the neuronal pool generating the rhythms is involved in a particular task, e.g. movement or processing of information. Pathological EEG rhythms can be observed in neurological disorders where the cortex is involved.

In general, the power in the frequency bands of the EEG increases when neurons generate synchronous rhythmic activity. During an "event" (for example a movement) neuronal firing desynchronizes and the amplitude of the frequency band is reduced: event related desynchronization (ERD). Frequencies that show modulations during a movement task are in the theta $(4-8 \mathrm{~Hz})$, alpha $(8-12 \mathrm{~Hz})$ and beta $(12-25 \mathrm{~Hz})$ range. Other rhythms may show modulations, as well. The power of theta frequencies increases during drowsiness or meditative stages of the brain. Alpha band oscillations play an active role in information processing and are suggested to be involved in inhibition and timing and suppression and selection (Klimesch 2012). The power of the signal in the beta frequency band may also increase during alertness, thinking and focus. The mu-rhythm is also found in the 9-13 Hz frequency band, but its profile over time is shaped like a character " $\mu$ " and it can be found over the motor cortex of the brain. Its amplitude reduces during planning and execution of a hand movement. A desynchronization of the murhythm over the sensorimotor cortex can also be found during observation, observation to imitate, and motor imagination of a movement (Cochin 1998, Hari 1998, Rizzolatti 2009).

However, the desynchronization of alpha- and beta-frequency bands during observation was found to be related to the ability to execute a movement (Orgs 2008). For example when professional dancers observed a dance movement, the power of these frequency bands was reduced when they observed a dance movement, but this reduction was not found in non-dancers who were not able to perform the movement (Orgs 2008). Before stroke, patients were able to execute "normal" movements, and it can be presumed that during observation of these movements the alpha and beta frequency bands was desynchronized. However, 
after stroke patients are not able anymore to execute the movement, and ERD is reduced in the affected hemisphere when compared with the unaffected hemisphere (Pfurtscheller 1980). Two evolutions have been observed over the period of time that the patient is recovering from a stroke: (1) a significant increase of the amplitude and area of ERD over the affected motor cortex, and (2) a decreasing trend of area and ERD over the non-affected motor cortex. It is suggested that in the acute stage after stroke, the non-affected hemisphere takes over the functions of the affected hemisphere. During stroke recovery, the affected hemisphere takes over its functions again and the dependence on the non-affected hemisphere decreases (Tangwiriyasakul 2014). The acquisition of the activity by the affected hemisphere is found to correlate with the degree of recovery of motor function (Marshall 2000, Feydy 2002, Ward 2003).

\subsubsection{Electromyography (EMG)}

Motor function impairments in stroke correlate with a decreased agonistic muscle activity and increased co-contraction (Hammond 1988, Chae ${ }^{a}$ 2002). To quantify muscle activation, surface electromyography (sEMG) can be used. sEMG measures the electrical activity of motor units at the muscle site. The firing of motor units results in a contraction of a muscle fiber. The amount of muscle contraction is a measure for the amount of motor units firing. The power (root mean square: RMS) of the EMG-signal measured by SEMG can be used to quantify the intensity of contraction of a muscle.

During a movement agonistic and antagonistic muscles are active. During flexion of the wrist for example, the flexors of the lower arm (agonists) are active, but also the extensors of the lower arm (antagonists). Co-contraction is the simultaneous activation of antagonist muscles, and can be quantified by the ratio between agonistic and antagonistic activity

After stroke, the co-contraction is increased, the antagonistic muscle activity is increased and/or the agonistic activity decreased. The remaining impairments of the upper limbs in chronic stroke are found to correlate with muscle weakness and with the degree of antagonistic muscle activity. Muscle activity can be measured by calculating the RMS of the EMG during maximal voluntary contraction in the agonist and antagonistic muscle. It was found that an increased antagonistic muscle activity and a decreased agonistic muscle activity correlate with the impairments (Hammond 1988, Chae ${ }^{\mathrm{a}} 2002$, Chae $\mathrm{e}^{\mathrm{b}}$ 2002). The abnormal contraction patterns cause movements to be rigid, or impossible to perform. Therefore, to decrease the physical impairments, it is important to increase the contraction and decrease the co-contraction. 


\subsection{Conclusion}

Depending on the region of the infarct a stroke leaves the patient suffering from highly invalidating impairments with significant impact on the activities of daily living. Rehabilitation therapies are focused on patients relearning or compensating for their lost abilities through exercise. These therapies are physically very intensive for patients who often concurrently suffer from cognitive impairments and psychological trauma. Additionally, these therapies are time consuming for the patients and for the therapist. In most cases, patients still suffer from motor impairments after therapy.

The administration of psychoactive drugs might facilitate the processes in the brain inducing recovery of motor function. In addition, the inclusion of movement imagination to the established rehabilitation program might be of additional value to regain motor function. Although results of these studies are promising, a relation between the cortical effects and the effects on the motor outcome has never been studied. Most of the previous studies examined the effects of motor function or the effects on cortical processes separately, and the relation between the effects of the interventions cannot be directly compared. By examining the relationship between changes of the event related (de)synchronization of the EEG and the power of the EMG in combination with the motor function processes contributing to potential improvement of the motor function might be better understood.

\subsection{Objectives and outline of the thesis}

The main objective of this thesis is to study the possibility to improve motor function after stroke by trying to enhance neuronal processes presumed to be involved in stroke recovery. We focus on two approaches, i.e. i) the use of psychoactive drugs and ii) the use of imagination of a movement.

We studied the potential contribution of these two approaches to stroke recovery by exploring the impact on cortical activity, muscle activation and motor function. When the action of these interventions is better understood and shown to be potentially relevant, a next step would be to explore the effects in a clinical trial.

Chapter 2 reviews studies investigating effects of psychoactive drugs on motor function after stroke. Previous studies examined several kinds of psychoactive drugs, affecting the amounts of different kinds of neurotransmitters in the brain. An overview of the range of psychoactive drugs is given in combination with the neurotransmitters they affect.

Based on the results in chapter 2, we decided to explore the effects of a single dose of fluoxetine $20 \mathrm{mg}$ when administered to chronic stroke patients (Chapter 3). 
With former studies showing that motor function is affected after a single dose of fluoxetine $20 \mathrm{mg}$, in Chapter 3 we examine the hypothesis that muscle activation patters will also be affected by the same dose in combination with changed motor function.

If fluoxetine improves the recovery of motor function after stroke, a relation is expected between the changed cortical activations and motor function. Motor function changes when muscle activation patterns change. Chapter 4 presents our findings on the relations between these physiological effects.

Motor imagination and movement observation comprise different concepts. Motor imagination can be defined as "the covert cognitive process of imagining a movement of your own body(-part) without actually moving that body(-part)" (De Vries 2007). Movement observation is defined as the perception of the actions of others. To define which of these concepts has the largest potential to induce neuroplasticity after stroke, differences are first tested in healthy subjects (chapter $5)$.

Chapter 6 presents the results of our efforts to examine effects of fluoxetine on cortical activity patterns during the execution of a movement and during imagination of a movement in chronic stroke patients. In this way, a possible reinforcement of fluoxetine on a therapy using imagination is explored. 


\subsection{References}

Adams HP Jr, Brott TG, Crowell RM, Fulran AJ, Gomez CR, Grotta J, Helgason CM, Marler JR, Woolson RF, Zivin JA et al. Guidelines for the management of patients with acute ischemic stroke. A statement for healthcare professionals from a special writing group of the Stroke Council, American Heart Association. 1994. Stroke 25:1901-1914

Astrup J, Siesjo BK, Symon L. Thresholds in cerebral ischemia-the ischemic penumbra. Stroke. 1981;12:723-725.

Astrup J, Symon L, Branston NM, Lassen NA. Cortical evoked potential and extracellular Kand H-at critical levels of brain ischemia. Stroke. 1977;8:51-57.

Babiloni C, Babiloni F, Carducci F, Cincotti F, Cocozza G, Del PC, et al. Human cortical electrencephalography (EEG) rhythms during the observation of simple aimless movements: a high-resolution EEG study. Neuroimage 2002;17:559-572

Barbay S, Nudo RJ (2009) The effects of amphetamine on recovery of function in animal models of cerebral injury: A critical appraisal. NeuroRehabilitation 25: 5-17.

Belayev L, Liu Y, Zhao W, Busto R, Ginsberg MD. Human albumin therapy of acute ischemic stroke. Marked neuroprotective efficacy at moderate doses and with a broad therapeutic window. Stroke 2001: 32; 553-560

Biernaskie J, Szymanska A, Windle V, Corbett D Bihemispheric contribution to functional motor recovery of the affected forelimb following focal ischemic brain injury in rats. Eur J Neurosci 200521 989-999

Boyeson MG, Feeney DM (1990) Intraventricular norepinephrine facilitates motor recovery following sensorimotor cortex injury. Pharmacol Biochem Behav 35: 497-501.

Buccino G. Action observation treatment: a novel tool in neurorehabilitation. 2014 Philos Trans R Soc Lond B Biol Sci.28;369

Calmels C, Holmes P, Jarry G, Leveque JM, Hars M, Stam CJ. Cortical activity prior to, and during, observation and execution of sequential finger movements. Brain Topogr 2006;19:77-88

Campos F, Blanco M, Barral D, Agulla J, Ramos-Cabrer P, Castillo J. Influence of temperature on ischemic brain: basic and clinical principles. Neurochem in 2012: 60;495-505

Carmichael ST. Plasticity of cortical projections after stroke. Neuroscientist 2003;9:64-75

Chaea J, Yang G, Park B, Labatia I. Muscle weakness and co-contraction in upper limb hemiparesis: relationship to motor impairment and physical disability. Neurorehabil Neural Repair. 2002;16:241-248.

Chae $^{\mathrm{b}}$ J, Yang G, Park B, Labatia I. Delay in initiation and termination of muscle contraction, motor impairment, and physical disability in upper limb hemiparesis. Muscle \& Nerve. 2002;25:568-575. 
Chen R, Cohen LG, Hallett M. Nervous system reorganization following injury. Neuroscience 2002;111:761-773

Chen, J., Fredrikson, V., Ding, Y., Cheng, H., Wang, N., Ling, F., et al. (2013). Enhanced neuroprotection by local intra-arterial infusion of human albumin solution and local hypothermia. Stroke 44, 260-262.

Cochin S, Barthelemy C, Lejeune B, Roux S, Martineau J. Perception of motion and qEEG activity in human adults. Electroencephalogr. Clin. Neurophysiol. 1998. 107:287-95

Cochin S, Barthelemy C, Roux S, Martineau J. Observation and execution of movement: similarities demonstrated by quantified electroencephalography. Eur J Neurosci 1999;11:1839-182

DeFazio, R. A., Zhao, W., Deng, X., Obenaus, A., \& Ginsberg, M.D. (2012). Albumin therapy enhances collateral perfusion after laser-induced middle cerebral artery branch occlusion: a laser speckle contrast flowstudy. J Cereb Blood FlowMetab 32, 2012-2022.

De Vries S, Mulder T. Motor imagery and stroke rehabilitation: a critical discussion. J Rehabil Med 2007;39:5-13

Doyle KP, Simon RP, Stenzel-Poore MP. Neuropharmacology- Special issue on cerebral ischemia mechanisms of ischemic brain damage - review article. Neuropharmacology. 2008 $55: 310-318$

Dreier JP. The role of spreading depression, spreading depolarization and spreading ischemia in neurological disease. 2011. Nature Medicine, 17:439-447

Erecinska M, Thoresen M, Silver IA. Effects of hypothermia on energy metabolism in Mammalian central nervous system. J Cereb Blood Flow Metab 2003: 23;513-530

Ertelt D, Small S, Solodkin A, Dettmers C, McNamara A, Binkofski F, et al. Action observation has a positive impact on rehabilitation of motor deficits after stroke. Neuroimage 2007;36 suppl2: T164-T173

Fan, X., Lo, E. H., \& Wang, X. Effects of minocycline plus tissue plasminogen activator combination therapy after focal embolic stroke in type 1 diabetic rats. Stroke 2013 44, 745752.

Feydy A, Carlier R, Roby-Brami A Bussel B, Cazalis F, Pierot L et al Longitudinal study of motor recovery after stroke: recruitment and focusing of brain activation. 2002 stroke: 33; $1610-1617$

Franceschini M, Agosti M, Cantagallo A, Sale P, Mancuso M, Buccino G. Mirror neurons: action observation treatment as a tool in stroke rehabilitation. Eur J Phys Rehabil Med 2010 46:517-523

Grade C, Redford B, Chrostowski J, Toussaint L, Blackwell B. Methylphenidate in early poststroke recovery: A double-blind, placebo-controlled study. Arch Phys Med Rehabil 1998; 79: 1047-1050. 
Gu Q. Neuromodulatory transmitter systems in the cortex and their role in cortical plasticity. Neuroscience 2002;111:814-835

Hakim AM. The cerebral ischemic penumbra.; Can J Neurol Sci 1987 14:557-559

Hammond M, Fitts S, Kraft G, Nutter P, Trotter M, Robinson L. Co-contraction in the hemiparetic forearm: quantitative EMG evaluation. Arch Phys Med Rehabil. 1988;69:348351.

Hari R, Forss N, Avikainen S, Kirveskari S, Salenius S, Rizzolatti G. Activation of human primary motor cortex during action observation: a neuromagnetic study. Proc. Natl. Acad. Sci. USA 1998 95:15061-65

Hasbani MJ, Underhill SM, De Erausquin GB, Goldberg MP. Synapse loss and regeneration: a mechanism for functional decline and recovery after cerebral ischemia? The Neuroscientist 2000: 6:110-119

Hofmeijer J, Van Putten MJAM. Ischemic cerebral damage: an appraisal of synaptic failure. Stroke 2012 43:607-615

Holmes GL and Khazipov R Basic neurophysiology and the cortical basis of EEG, in The Clinical Neurophysiology Primer A.S. Blum, Editor. Humana Press: Totowa, New Jersey.2007: 29-31

Ilic T, Korchounov A, Ziemann U. Complex modulation of human motor cortex excitability by the specific serotonin re-uptake inhibitor sertraline. Neuroscience Letters 2002;319:116120.

Imai, H., Graham, D. I., Masayasu, H., \& Macrae, I. M. Antioxidant ebselen reduces oxidative damage in focal cerebral ischemia. Free Radic Biol Med 2003 34, 56-63.

Jacobs B, Fornal C. Activity of serotonergic neurons in behaving animals. Neuropsychopharmacology 1999;21:9S-15S.

Kandel E, Schwartz J, Jessell T. Principles of neural science. ed. McGraw-Hill Companies, 2000:

Karibe H, Zarow GJ, Graham SH, Weinstein PR Mild intraischemic hypothermia reduces postischemic hypoperfusion, delayed postischemic hypoperfusion, blood-brain barrier disruption, brain edema, and neuronal damage volume after temporary focal cerebral ischemia in rats. J Cereb Blood Flow Metab 1994: 14; 620-327

Kaufmann AM, Firlik AD, Fukui MB, Wechsler LR, Jungries CA, Yonas H. Ischemic core and penumbra in human stroke. Stroke 1999 30: 93-99

Kleim, J. A. \& Jones, T. A. Principles of experience dependent neural plasticity: implications for rehabilitation after brain damage. 2008 J. Speech Lang. Hear. Res. 51, S225-S239

Klimesch W. $\alpha$-band oscillations, attention, and controlled access to stored information. 2012 Trends Cogn Sci 16:606-617 
Lapchak, P. A., \& Zivin, J. A. (2003). Ebselen, a seleno-organic antioxidant, is neuroprotective after embolic strokes in rabbits: synergism with low-dose tissue plasminogen activator. Stroke 34, 2013-2018.

Lapchak, P. A. A critical assessment of edaravone acute ischemic stroke efficacy trials: is edaravone an effective neuroprotective therapy? Expert Opin Pharmacother 2010 11, 17531763.

Lauritzen, M., Dreier, J. P., Fabricius, M., Hartings, J. a, Graf, R., \& Strong, A. J. Clinical relevance of cortical spreading depression in neurological disorders: migraine, malignant stroke, subarachnoid and intracranial hemorrhage, and traumatic brain injury. Journal of Cerebral Blood Flow and Metabolism : Official Journal of the International Society of Cerebral Blood Flow and Metabolism, 2011 31(1), 17-35.

Laver KE, George S, Thomas S, Deutsch JE, Crotty M. Virtual reality for stroke rehabilitation. Cochrane Database Syst Rev 2015: 12;2

Loubinoux I, Boulanouar K, Ranjeva J, Carel C, Berry I, Rascol O. Cerebral functional magnetic resonance imaging activation modulated by a single dose of the monomine neurotransmission enhancers fluoxetine and fenozolone during hand sensorimotor tasks. Journal of Cerebral Blood Flow and Metabolism 1999;19:1365-1375.

Loubinoux I, Pariente J, Boulanouar K, et al. A Single Dose of the Serotonin Neurotransmission Agonist Paroxetine Enhances Motor Output: Double-Blind, PlaceboControlled, fMRI Study in Healthy Subjects. NeuroImage 2002;15:26-36.

Marshall RS, Perera GM, Lazar RM, Krakauer JW, Constantine RC, DeLaPaz RL Evolution of cortical activation during recovery from corticospinal tract infarction. 2000. Stroke 31656 661

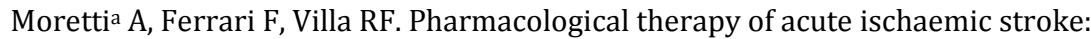
Achievements and problems. Pharmacol Ther. 2015; 153:79-89

Morettib A, Ferrari F, Villa RF. Neuroprotection for ischaemic stroke: current status and challenges. Pharmacology \& Therapeutics 2015; 146:23-34

Murphy TH, Corbett, D. Plasticity during stroke recovery: from synapse to behavior. Nat Rev Neurosci 2009; 10:861-872.

Orgs G, Dombrowski JH, Heil M, Jansen-Osmann P. Expertise in dance modulates alpha/beta event-related desynchronization during action observation. 2008. Eur J Neurosci 27:33803384 .

Page SJ, Levine P, Leonard A. Mental practice in chronic stroke: results of a randomized, placebo-controlled trial. Stroke 2007;38:1293-1297

Pascual-Leone A, Amedi A, Fregni F, Merabet LB. The plastic human brain cortex. 2005 Annu Rev Neurosci 28:377-401

Pleger B, Schwenkreis P, Grünberg C, Malin J-P, Tegenthoff M. Fluoxetine facilitates usedependent excitability of human primary motor cortex. Clinical Neurophysiology 2004;115:2157-2163. 
Pfurtscheller G, Aranibar A, Wege W. 1980 Changes in central EEG activity in relation to voluntary movement. II. Hemiplegic patients Prog Brain Res 54:491-495

Pfurtscheller G, Brunner C, Schlögl A, Lopes da Silva FH. Mu rhythm (de)synchronization and EEG single-trial classification of different motor imagery tasks. 2006 Neuroimage 15;31:153-159

Rizzolatti G, Fabbri-Destro M, Cattaneo L. Mirror neurons and their clinical relevance. 2009 Nat Clin Pract Neurol 5 24-34

Rizzolatti G, Laila Craighero. The mirror-neuron system. 2004 Annu Rev Neurosci 27:169192

Rossini PM, Calautti C, Pauri F, Baron JC. Post-stroke plastic reorganization in the adult brain. Lancet Neurol 2003 2:493-502

Rossini PM, Dal Forno G. Integrated technology for evaluation of brain function and neuronal plasticity. Phys Med Rehabil Clin N Am 2004;25:263-306

Ruscher K, Kuric E, Wieloch T. Levodopa treatment improves functional recovery after experimental stroke. Stroke 2012 43: 507-513.

Saver JL, Starkman S, Eckstein M, Stratton S, Pratt F, Hamilton S et al. Methodology of the field administration of stroke therapy - Magnesium (FAST-MAG) phase 3 trial: Part 2 prehospital study methods. In J stroke 2014: 42; 825-829

Sawaki L et al. Constraint-induced movement therapy results in increased motor map area in subjects 3 to 9 months after stroke. Neurorehabil Neural Repair 2008: 22;505-513

Symon L. The relationship between CBF, evoked potentials and the clinical features in cerebral ischaemia. Acta Neurol Scand Suppl. 1980; 78:175-190.

Tangwiriyasakul C, Verhagen R Rutten WLC, Van Putten MJAM. Temporal evolution of event-related desynchronization in acute stroke: a pilot study. 2014 Clin Neurophysiol 125: $1112-1120$

Tardy J, Pariente J, Leger A, Dechaumont-Palacin S, Gerdelat A, Guiraud V, Conchou F, Albucher J-F, Marque, Franceries X, Cognard C, Rascol O, Chollet F, Loubinoux I.

Methylphenidate modulates cerebral post-stroke reorganization. Neuroimage 2006 33: 913-922.

Van Putten MJAM. Essentials of neurophysiology \pm Basic concepts and clinical applications for scientists and engineers. 1st ed. Series in Biomedical Engineering, ed. J.H. Nagel. 2009, Berlin Heidelberg: Springer-Verlag

Ward NS, Brown MM, Thompson AJ, Frackowiak RS. Neural correlates of outcome after stroke: a cross-sectional fMRI study. 2003 Brain 126 1430-1448

Weikop P, Yoshitake T, Kehr J. Differential effects of adjunctive methylphenidate and citalopram on extracellular levels of serotonin, norepinephrine and dopamine in the rat brain. Eur Neuropsychopharmacol 2007 17: 658-671. 
Chapter 1

Yenary MA, Han HA. Neuroprotective mechanisms of hypothermia in brain ischaemia. Nat Rev neurosci 2012: 13;267-278

Zandt, B. J., Ten Haken, B., \& van Putten, M. J. A. M. Diffusing Substances during Spreading Depolarization: Analytical Expressions for Propagation Speed, Triggering, and Concentration Time Courses. The Journal of Neuroscience : The Official Journal of the Society for Neuroscience, 2013 33(14), 5915-23. 


\section{CHAPTER 2}

\section{The clinical use of drugs influencing neurotransmitters in the brain to promote motor recovery after stroke: a Cochrane systematic review}

Berends HI, Nijlant JM, Movig KLL, Van Putten JM, Jannink MJA, IJzerman MJ Eur J Phys Rehabil Med 2009 45: 621-630 


\subsection{Abstract}

Introduction

The objective of this review is to compare and to discuss the results of studies that investigated the ability of drugs to improve motor recovery after stroke by influencing dopamine, norepinephrine, or serotonin concentrations in the brain.

Data sources

A systematic literature search up to January 2009 was conducted in MEDLINE, Pubmed, EMBASE and in the database of the Cochrane Stroke Group Trial Register. In addition, the literature reference lists of the relevant publications were checked. Review Methods

The literature search was conducted in order to identify randomized controlled trials focusing on the effects of drugs on motor recovery after stroke. In order to structure the data, motor recovery was sub-divided into motor control and motor function. The methodological quality of the studies was also assessed.

$\underline{\text { Results }}$

Six studies, investigating the effects of 7 different kinds of drugs were included. Methodological scores varied between 10 and 14 (max 19). Motor control was not influenced by any of the drugs. Motor function improved in patients treated with methylphenidate, trazodone, and nortriptyline. Results for fluoxetine and levodopa were contradicting.

Conclusion

There is insufficient evidence to conclude that neuromodulating drugs targeting serotonin, norepinephrine, or dopamine influence motor recovery after stroke.

Key Words: motor recovery, stroke, pharmacotherapy, neurotransmitters 


\subsection{Introduction}

A stroke or a cerebrovascular accident (CVA) is a sudden ischemic or hemorrhagic disturbance in the blood supply to the brain that results in complete or partial loss of brain function. In industrialized countries, it is the leading cause of disability, and one of the major consequences of a disturbed co-ordination of (fine) motor tasks. Therefore the (re)learning of motor function plays an important role in the rehabilitation after a stroke. After the initial recovery, cortical plasticity is presumed to be important for the improvement of motor function. Cortical plasticity is established by several mechanisms, including unmasking of silent synapses, sprouting of nonaffected fibers, regeneration of axons, and long-term potentiation (LTP) ${ }^{1,2,3,4}$. Unmasking of previously silent synapses is promoted by an increased excitability of the brain ${ }^{5}$. In sprouting, new synapses are formed and in LTP an increase in synaptic strength provides more stable changes in the horizontal connections within the primary motor cortex ${ }^{6,7}$.

The release of local neurotransmitters (acetylcholine, noradrenalin, serotonin, dopamine, and histamine) is thought to influence cortical plasticity, since an increased amount of these neurotransmitters enhances the excitatory input of glutamate in cortical neurons. This enhanced input of glutamate in turn facilitates N-methyl-D-aspartate (NMDA) receptor activation. When intracellular events following NMDA receptor activation overcome a threshold, synaptic modifications occur $^{2}$. The facilitation of NMDA receptor activation might therefore promote these synaptic modifications, which are important in cortical plasticity. Previous studies have found a relation between these neurological changes and motor control and functional abilities ${ }^{6,8}$. Therefore, it is hypothesized that drugs that increase the amount of neurotransmitters, will facilitate cortical plasticity, and thereby also motor recovery after stroke.

Several drugs influence the amounts of neurotransmitters in the brain. Dextro- and methamphetamine are examples of drugs that increase the amounts of dopamine, norepinephrine, and serotonin in the synaptic cleft by inhibiting the re-uptake of these neurotransmitters into the presynaptic terminal. Studies investigating the effects of these amphetamines on motor recovery after stroke have been extensively summarized in a previous review ${ }^{9}$. This review suggested that motor and language function might improve with amphetamine-treatment. However, no effect of treatment on activities of daily living, neurological function, or depression was found. Other studies on amphetamines show insufficient evidence of a facilitating effect of dextroamphetamines on motor function ${ }^{10,11,12}$. Because of these previously published data, amphetamines are not taken into account in the present review. Recently, Rösser and Floël published a descriptive overview of the conclusions of studies examining pharmacological enhancements of motor recovery after stroke ${ }^{13}$. This study concluded that preliminary data are promising, and larger clinical trials are needed. However, this review only considered the conclusions of the studies and did not take the quality into account. To come to a 
deeper understanding of the way of action of the pharmacology and the relation with motor recovery, the present review systematically analyzes the results of studies examining pharmacological treatment in stroke aimed to improve motor function.

Several drugs have a rather selective influence on neurotransmitters in the brain. Levodopa (L-dopa) ${ }^{14,15}$, increases dopamine concentrations in the brain. It is the metabolic precursor of dopamine, and it is able to cross the blood-brain barrier. In the brain it is converted into dopamine. Methylphenidate ${ }^{16}$ also increases the amount of dopamine in the brain by blocking the re-uptake of dopamine (and also norepinephrine) into the presynaptic neurons and by increasing the release of these neurotransmitters into the extraneuronal space. Dopamine is a neurotransmitter that most likely regulates plasticity in neuronal circuits, and it reduces or facilitates communication among neurons ${ }^{2}$. Dopamine is involved in the regulation of motor control and the learning of motor programs and habits. It is also required for voluntary movement ${ }^{17,18}$.

Antidepressants, such as desipramine ${ }^{19}$, maprotilinem ${ }^{20}$ and nortriptyline ${ }^{21}$ are drugs that predominantly influence the amount of norepinephrine in the brain. Desipramine is a tricyclic antidepressant, and it is suggested that it increases norepinephrine amounts by blocking the reuptake from the synapses in the central nervous system. Maprotiline is an antidepressant which non-selectively inhibits monoamine re-uptake, resulting in an inhibition of re-uptake of norepinephrine in the presynaptic neurons. Nortriptyline also blocks the uptake of norepinephrine in the presynaptic neurons. Norepinephrine is the predominant transmitter in the autonomic nervous system, and plays an important role in learning and memory ${ }^{18}$. Fluoxetine and trazodone are drugs that influence the amount of serotonin in the brain. Fluoxetine is a selective serotonin re-uptake inhibitor which increase serotonin amounts in the synaptic cleft by inhibiting the reuptake of serotonin by the presynaptic neuron. Besides its effect on serotonin-receptors, trazodone also affects histamine, and $\alpha 1$ adrenoreceptors and possibly inhibits the action of recombinant T-type channels ${ }^{22}$. Serotonin is thought to facilitate motor output ${ }^{2,23}$. It has also been hypothesized that serotonergic neurons play an auxiliary role in co-ordinating appropriate autonomic and neuroendocrine outputs to the ongoing tonic or repetitive motor activity ${ }^{23}$.

The objective of this research is to systematically review the results of studies that investigated the ability of drugs to improve motor recovery by influencing dopamine, norepinephrine, or serotonin concentrations in the brain. 


\subsection{Methods}

Literature search

A systematic literature search up to January 2009 was conducted in MEDLINE, Pubmed, EMBASE and in the database of the Cochrane Stroke Group Trial Register. The following key words were used in these searches: cerebrovascular disorders, rehabilitation, activities of daily living, treatment outcome, motor skills, motor activity, movement, pharmacology, neurotransmitter uptake inhibitors, and neurotransmitter agents. The Pubmed search is described in Appendix 1 and was adapted to suit the other databases. In addition, the literature reference lists of the relevant publications were carefully checked.

\section{$\underline{\text { Selection criteria }}$}

Studies that met the following criteria were included:

1) completed, randomized, controlled, published clinical trials in humans, 2) the drug tested had to affect dopamine, norepinephrine, or serotonin amounts in the brain, 3) the primary outcome measure had to be related to motor recovery after stroke, studies only measuring with the Barthel Index, or the Rankin score were excluded, 4) the drug had to be administered during a prolonged period of at least 2 weeks, 5) full-length publication in English, German, or Dutch. Case reports were not included.

\section{Assessment of methodological quality}

To evaluate the methodological quality of the studies, we used the "Criteria List for the Methodologic Quality Assessment" of Van Tulder ${ }^{24}$. This list consists of internal validity criteria, descriptive criteria, and statistical criteria, assessed by 19 questions which could be answered with "Yes", "No", or "Don't know" (Appendix 2). Each positive answer scored 1 point and each negative, or unclear answer scored 0 points. Each trial was evaluated by three reviewers independently (HB, JN, MIJ), and any disagreement between the reviewers was cross-checked and solved by discussion. 


\section{Data-extraction and analysis}

In order to analyze the contents of the selected studies, study characteristics were extracted from the manuscripts (table 1). This information includes:

1. the methodological quality of the study

2. the intervention(s) used in the study

3. the descriptive features of the subjects (age, time since stroke, kind of stroke)

4. the number of included patients

5. the motor scores prior to the intervention and the motor scores after intervention

6. the side-effects and drop-out

7. decrease or increase of depression during study period.

In defining the clinical outcome (motor recovery) we separated motor control from motor function. Motor control is defined as the ability to use the muscles in order to accomplish a task, and can be measured (among other methods) with the FuglMeyer motor assessment (FM). On the other hand, motor function is defined as the ability to function in daily life, which can be measured (among other methods) with the Barthel Index (BI), the motor section of the Hemispheric Stroke Scale (HSS ${ }_{\text {motor }}$ ), the Functional Independence Measure (FIM), Rivermead Motor Assessment (RMA), and the Johns Hopkins Functioning Inventory (JHFI). When interpreting the data we used these categories.

The differences in the changes in motor control and motor function before and after the intervention between the medication and placebo groups were considered to be significant if $\mathrm{p}<0.05$.

In the assessment of the drugs, the safety and the possible side-effects of the drug were taken into account.

\subsection{Results}

Four publications ${ }^{14,15,19,20}$ fulfilled all the selection criteria (Appendix 1) and were included in the review. Two more articles were retrieved via the reference lists ${ }^{16,21}$. The studies that are included are summarized in table 1.

In 6 studies, 7 different kinds of drugs were tested in 266 patients with 6 different outcome measures. In order to make a comparison despite the large number of variables, the outcome measures were grouped into measures of motor function or motor control. All studies investigated the motor function with the $\mathrm{HSS}_{\text {motor, }} \mathrm{BI}$, FIM, RMA, or JHFI. Motor control was measured in only three studies with the $\mathrm{FM}^{15,16,19}$.

Dam et a ${ }^{20}$ found a significant improvement in motor function in all groups over time. However, according to the BI, motor function improved significantly more in patients treated with fluoxetine than in patients treated with maprotiline. The $\mathrm{HSS}_{\text {motor }}$ score found no significant differences between groups, but the $\mathrm{HSS}_{\text {gait }}$ 
scores did. Motor control was not measured. No significant differences were found between any of the treatment groups and the placebo group.

Grade et $a l^{16}$ found that patients treated with methylphenidate had a better recovery of motor function (FIM), compared to the placebo groups, with no effect on motor control (FM). In this study, the patients receiving methylphenidate showed a decreased degree of depression, compared to the study group receiving placebo.

Scheidtmann et al $^{14}$ found that patients receiving levodopa had significantly more improvement in motor function (RMA) than those receiving placebo. This improvement was maintained, or increased even further in both groups for at least 3 weeks after the end of the treatment. In this study, depression was not measured. However, levodopa is not an antidepressant.

Miyai et al ${ }^{19}$ found that motor function improved more in patients who were treated with trazodone and fluoxetine than in patients treated with desipramine (FIM). The differences in motor function were mainly due to sub-scores for ADL and mobility, and not cognition sub-scores. There were no significant differences in improvement in motor control (FM). All three of the drugs that were tested were found to have the same effect on depression.

Robinson et $a l^{21}$ studied the effects of fluoxetine, nortriptyline and placebo on motor function in depressed and non-depressed patients separately. In the group of depressed patients, contradicting results were found between two motor function scales. According to the BI, they found a significantly greater improvement in motor function in patients who were treated with nortriptyline or placebo than in patients who were treated with fluoxetine. The JHFI scores did not differ between the treatments in the depressed patient group. The patients treated with nortriptyline had a significantly greater decline in HDRS (Hamilton rating scale for depression) than the patients treated with placebo or fluoxetine. In the group of non-depressed patients, no differences in motor function or depression were found between the treatments.

Finally, Sonde et al ${ }^{15}$ compared the effects of L-dopa alone, or in combination with amphetamines, with the effects of amphetamine alone and placebo. All patients improved significantly with regard to motor control (FM) and motor function (BI) over time. However, no additional increase was found in any of the treatment groups. The degree of depression was not measured.

The methodological scores are summarized in table 1 . The scores vary between 10 and 14 out of 19, which led to the conclusion that the methodological quality of all the studies was moderate. The most conspicuous aspects are the lack of comparability between the groups at baseline with regard to the most important prognostic indicators, compliance therapy, and the lack of avoidance of cointerventions. Except for the study of Dam et al ${ }^{20}$, all studies had at least one important prognostic indicator that differed between the treatment groups. None of the studies described compliance with the therapy, and co-interventions were 
not described in three studies ${ }^{15,16,21}$. In the study of Miyai et al ${ }^{19}$ co-interventions were not avoided. These aspects might have influenced the results of the studies.

Safety

Table 1 summarizes the described side-effects and the reasons for the drop-out. Because sub-acute stroke patients are susceptible for seizures, a second stroke, medical deterioration, and other medical problems, the drop-out rate in all of the studies was rather high. These reasons for drop-out were probably not due to the side-effects of the drug. However, in the treatment group receiving desipramine ${ }^{19}$ many of the reasons for drop-out (like drug rash, tachycardia or confusion) were probably due to side-effects of desipramine.

In several studies "refused treatment" is mentioned as a reason for drop-out. However, the reason for refusal is not mentioned, it could have been due to sideeffects, but also to other variables influencing participation. 
Influencing neurotransmitters to promote recovery, systematic review

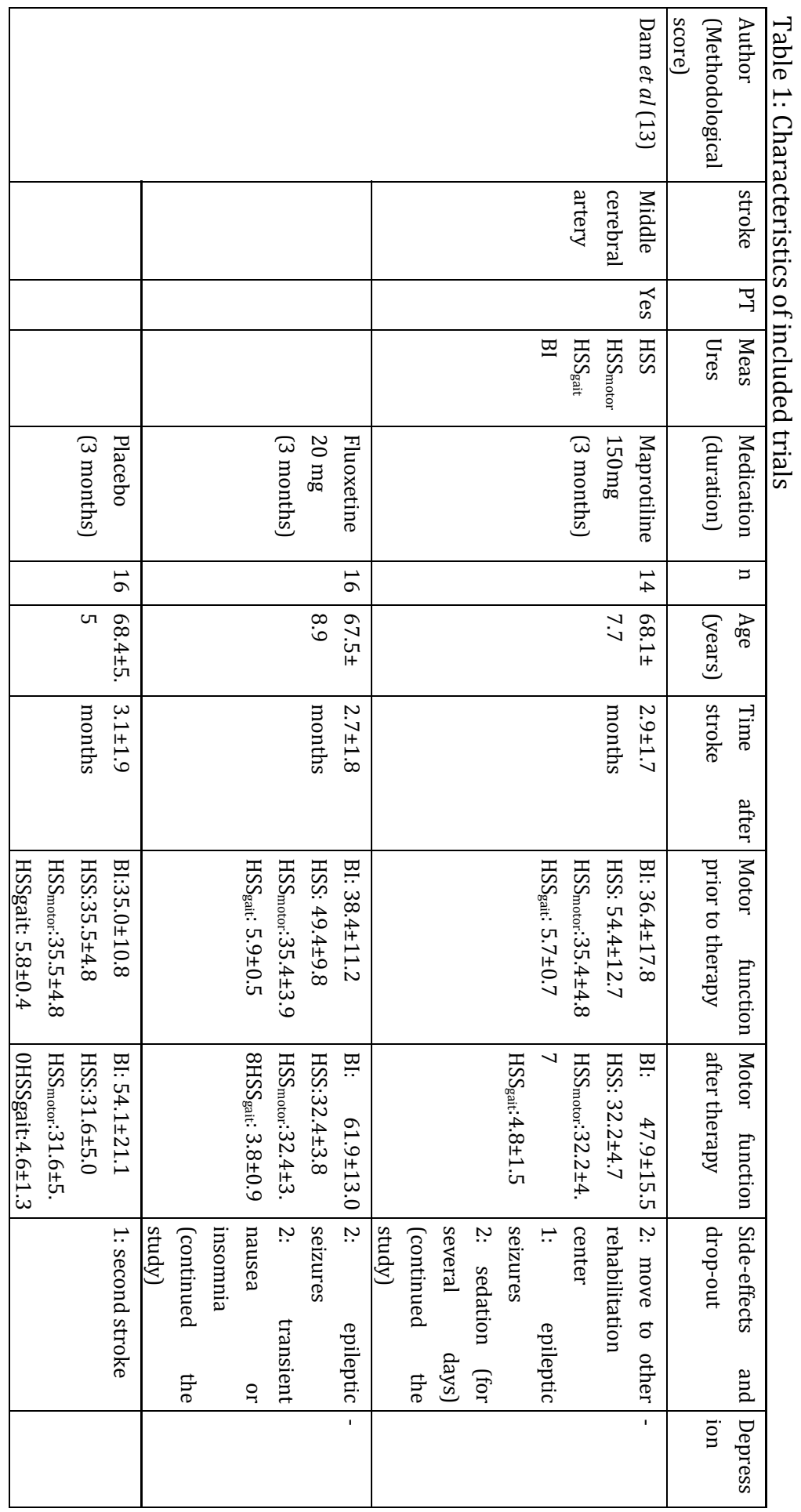




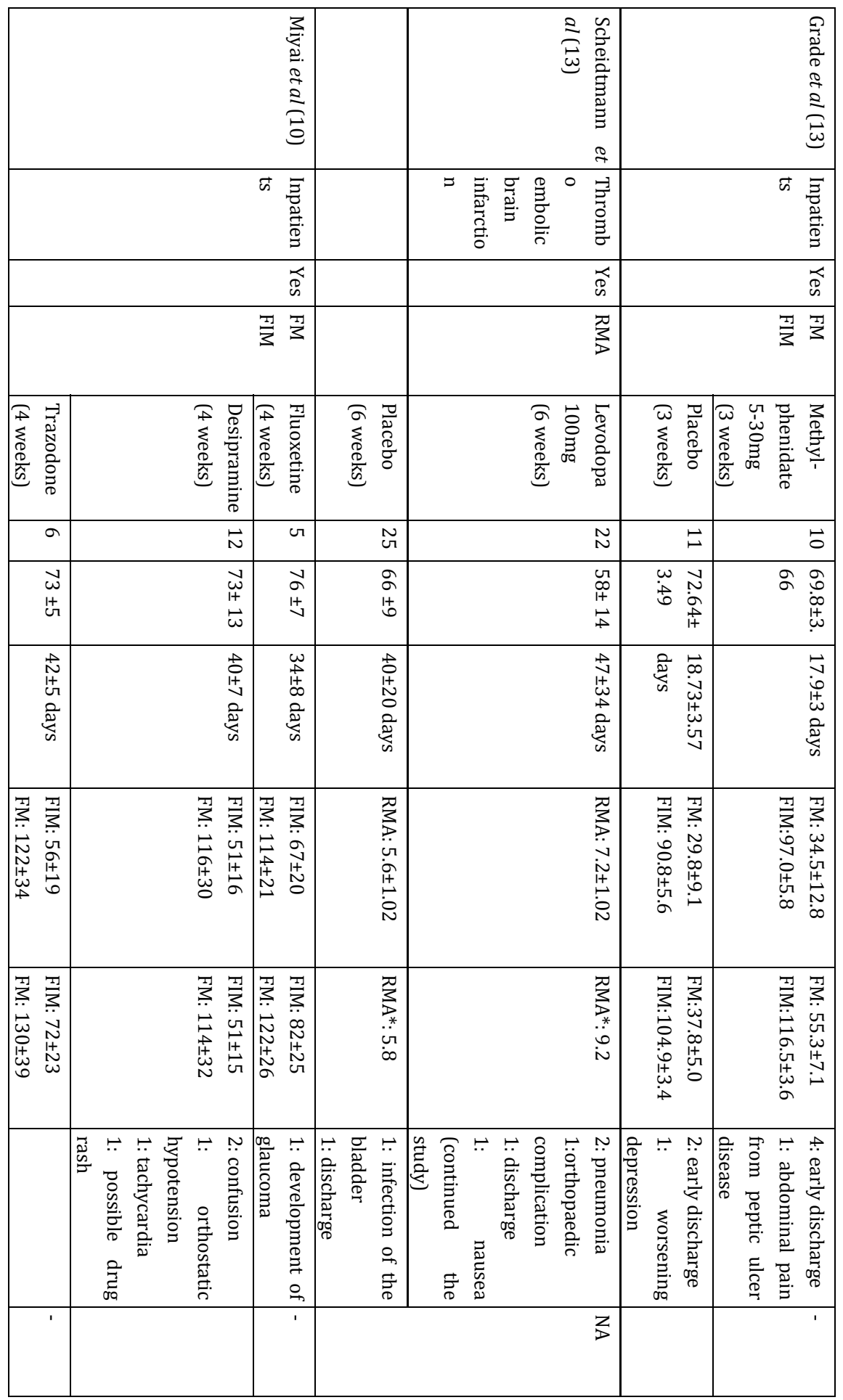




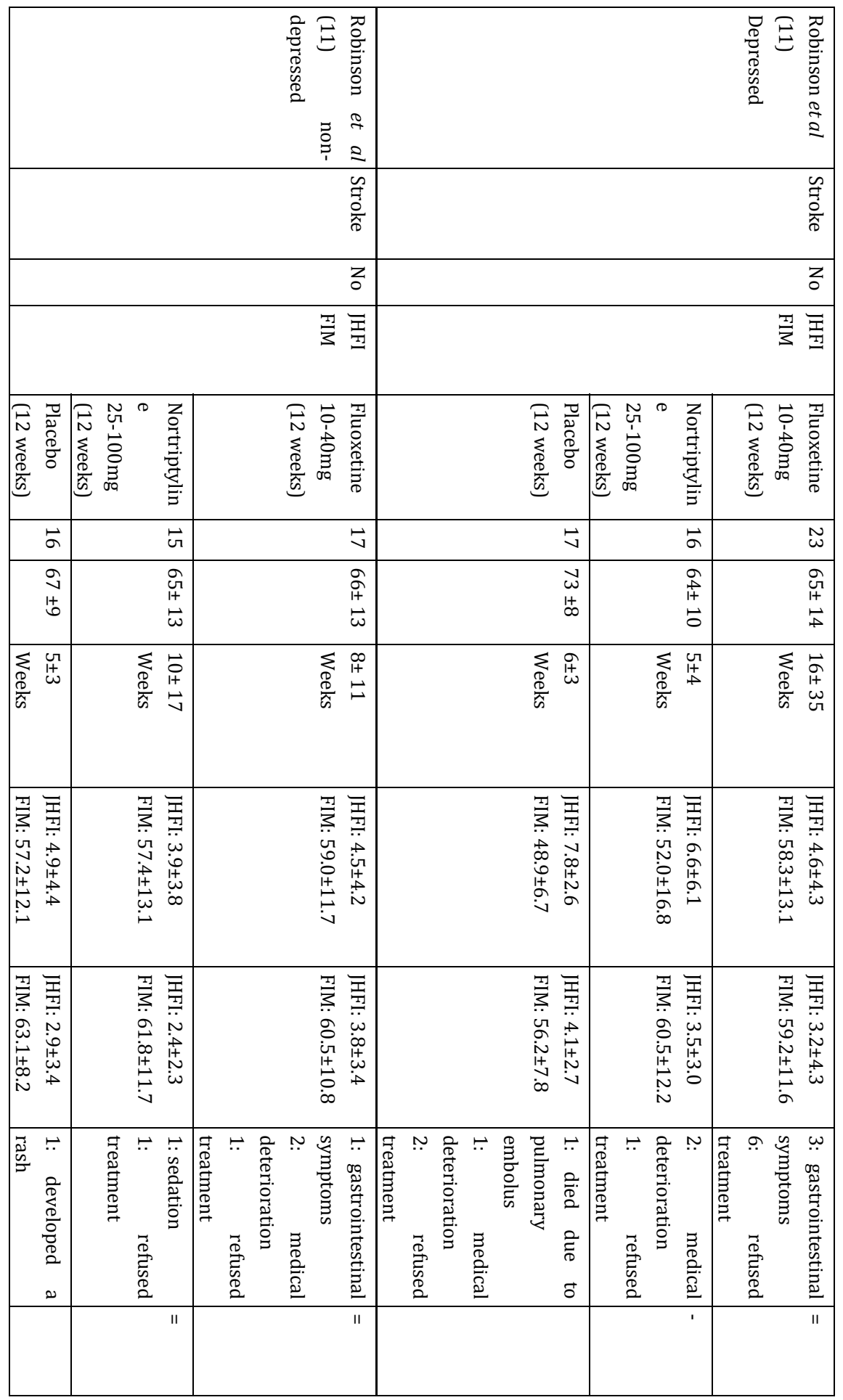




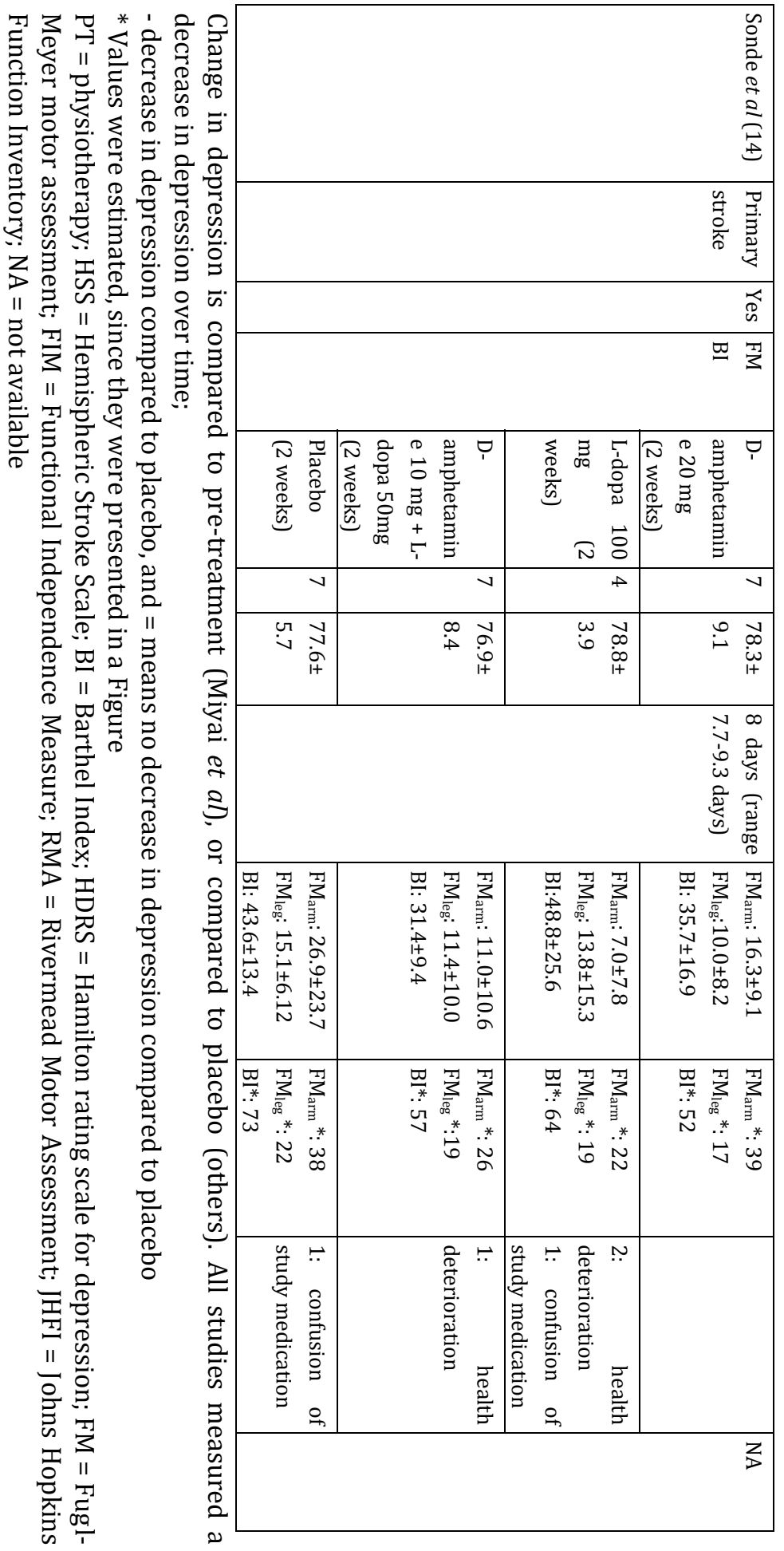




\subsection{Discussion}

In the present review 6 RCTs were found that assessed the effects of various drugs on motor recovery after stroke. To reduce the large number of variables, the outcome measures were sub-divided into measures of motor function and measures of motor control.

Motor control was not influenced by any of the drugs that were investigated in the studies. This is in contrast with the results of previous studies on the effects of a drug after a single dose or in healthy subjects. In most of these studies, motor control improved after the intake of a single dose of fluoxetine ${ }^{25}$, citalopram ${ }^{26}$, or reboxetine ${ }^{27}$ in chronic stroke patients, and after the intake of fluvoxamine in healthy subjects ${ }^{28,29}$. However one study found that a single levodopa dose was not sufficient to improve motor function in chronic stroke ${ }^{30}$. In addition, we found no effect of a single dose of fluoxetine on motor function or motor control, however, muscle activity during maximum voluntary force increased ${ }^{31}$.

Neurological studies also suggest an influence of drugs on motor control. Pariente et al found an increased activation in executive motor areas after fluoxetine intake in stroke patients ${ }^{25}$. Loubinoux found a modulated activation in the sensorimotor area in healthy subjects after the intake of fluoxetine, fenozolone and paroxetine ${ }^{32,33}$. The excitability of the sensorimotor cortex also increased after sertraline ${ }^{34}$ and fluoxetine ${ }^{35}$ intake in healthy subjects. In addition, two studies found a relationship between increased motor cortex excitability, decreased activation of the sensorimotor cortex and an improvement in the finger-tapping test score in healthy subjects ${ }^{36,37}$.

Motor function improved in the patient groups treated with methylphenidate, trazodone, and nortriptyline. The results for fluoxetine and levodopa were contradicting. According to the hypothesis that the effects of drugs on motor function are established by mediation of neurotransmitter concentrations, it can be expected that drugs influencing the same neurotransmitters have the same effect on motor function. However, no relationship was found between the affected neurotransmitter and change in motor function, since the drugs that were found to facilitate motor function (methylphenidate, trazodone, and nortriptyline) did not differ from the drugs that were not found to improve motor function (maprotiline and desipramine). For example, maprotiline and nortriptyline both influence norepinephrine amounts. Nortriptyline was found to improve motor function ${ }^{21}$, while maprotiline did not ${ }^{20}$. In addition to the influence of the drugs on dopamine, norepinephrine, or serotonin concentrations, the drugs influencing norepinephrine concentractions, also influence adrenergic propertie, since norepinephrine is a precursor of epinephrine. However, nor the results of drugs influencing epinephrine are consistent. The differences between the results of the studies might therefore not solely be explained by an increase in the different neurotransmitters in the brain. 
Probably, other variables such as cognitive impairments or depression interfere with the effects of the drugs in stroke patients during long-term administration. We found no studies that investigated their influence on motor control. However, depression $38,39,40$, neurological and cognitive factors are thought to influence the degree of motor function after a stroke ${ }^{41}$. Of the included studies, only two measured the influence of the drug on the cognition of the patients (Mini Mental State Examination $)^{16,21}$. Neither one of the studies found any reason to presume that cognitive factors influenced the outcomes.

However, changes in the degree of depression between the treatment groups did differ. Previous studies have shown that remission of depression was associated with a greater recovery of motor function ${ }^{40,41}$. Also, in the studies included in the present review, an improvement in motor function in all treatments groups followed an improvement in the degree of depression. Although Hackett and coworkers ${ }^{42}$ found no definitie evidence that antidepressants prevent depression or improve recovery after stroke, the degree of depression might act as an effectmodifier, and it could be suggested that a drug improves the motor function by decreasing the degree of depression.

In addition to depression, several other differences between the treatment groups might have influenced the results. A variable that differs between treatment groups is the site of stroke, which might influence motor recovery and possibly also the effect of the drug. The study of Dam et a ${ }^{20}$ is the only study that restricted the infarct location to a hemispheric middle cerebral artery. The other studies all included patients with a stroke in different areas of the brain. If patients with different sites of stroke are included, they should be equally distributed between the groups. However, in the study of Grade et al ${ }^{16}$ twice as many patients with cortical stroke were included in the placebo group than in the group receiving methylphenidate treatment. Also in the study of Miyai et al ${ }^{19}$ the site of stroke was not equally divided over the groups.

The interval between inclusion and stroke onset also differed between the groups. The degree of recovery depends largely on the time after the stroke -soon after a stroke the motor function is presumed to improve more than a few weeks or months after the stroke- therefore, it is important to balance the interval between inclusion and the stroke between the groups. Differences between these intervals make comparison between the studies hard. Sonde et al18 included patients 5 to 10 days after a stroke, while Dam et al ${ }^{20}$ included patients 3 months after stroke. Also, within some of the studies the interval between inclusion and the stroke differed between the treatment groups. In the study of Robinson et al21 (depressed patient group) the mean time after the stroke in the fluoxetine group was $16 \pm 35$ weeks, while the patients in the nortriptyline and placebo groups were included $5 \pm 4$ and $6 \pm 3$ weeks after the stroke, respectively. This could explain the differences found between the effects of fluoxetine, and nortriptyline and placebo. 
Another difference between the groups is the degree of impairment at the start of the trial. In the studies of Miyai et $a 1^{19}$, Robinson et $a l^{21}$ (depressed patients), Scheidtmann et al ${ }^{14}$ and Sonde $e t a l^{15}$, this motor impairment differed between the groups. When a group already has a high score for motor function, there is less potential to improve than in a group with a low initial score. The effect of the treatment can therefore not be compared between groups with different degrees of impairment.

The dosage of the drugs is also important when comparing studies. Loubinoux et al indicated that differences between doses influence the outcome ${ }^{33}$. The different results reported in the studies investigating fluoxetine might be due to this difference in dosage. None of the studies investigated the effects of different doses of the same drug.

The treatment of stroke patients with the drugs was found to be safe. However, the side-effects that did not result in drop-out were not described in three of the six studies. Only Dam et $a{ }^{20}$, Scheidtmann et $a l^{14}$, and Sonde et al ${ }^{15}$ described all sideeffects, including those that did not result in drop-out. Grade et a ${ }^{16}$ only reported a rating of the seriousness of the side-effects. In addition, some studies were unclear in their description of the side-effects, since no reasons were given for refusal of treatment, or for medical deterioration. The description of all side-effects is important, because these can impede motor recovery.

In the decision to administer a drug, the side-effects have to be taken into account. However, if the inclusion and exclusion criteria are formulated with care, no drug needs to be omitted from future studies because of its side-effects.

Although a positive trend is found in the relation between administration of pharmacology and motor function, the hypothesis that an increased amount of any of the serotonin, norephinephrine, or dopamine neurotransmitters will improve motor recovery after stroke can be neither confirmed nor rejected.

To gain a better understanding of the effects of the drugs influencing the neurotransmitter concentrations in the brain and their influence on motor rehabilitation, more basic research has to be done. The mechanisms behind the effects of drugs are attributed to the influence on various neurotransmitters, which are presumed to be relevant in cortical plasticity. To evaluate their clinical utility, the effects of these drugs on cortical, spinal, and muscle activity have to be correlated with changes in motor function and motor control. When these processes are better characterized and understood we may ultimately recommend specific drug treatment in post-stroke patients to improve motor recovery during rehabilitation. 


\section{Appendix 1}

Literature search, combination of keywords

\#1 Cerebrovascular disorders [MeSH] OR Cerebrovascular accident[MeSH] OR Stroke[MeSH]

\#2 Rehabilitation [MeSH] OR Rehabilitation [subheading] [MeSH] OR activities of daily living [MeSH] Or Treatment Outcome [MeSH]

\#3 Motor Skills [MeSH] OR Motor Activity [MeSH] OR Movement [MeSH]

\#4 Pharmacology [MeSH] OR Pharmacology [subheading] [MeSH] OR Pharmacology, clinical

\#5 Neurotransmitter uptake inhibitors [MeSH] OR Neurotransmitter Uptake inhibitors [pharmacological action] [MeSH] OR Dopamine Uptake Inhibitors [MeSH]

\#6 Neurotransmitter Agents [MeSH] OR neurotransmitter agents [pharmacological action]

Limits were studies in Humans, English, German, Dutch

\#1 AND \#2 AND \#3 AND \#4

\#1 AND \#2 AND \#3 AND \#5

\#1 AND \#2 AND \#3 AND \#6

\section{Appendix 2}

"Criteria List for the Methodologic Quality Assessment" of Van Tulder ${ }^{24}$

Patient selection

a. Were eligibility criteria specified?

Yes/No/Don't know

b. Treatment allocation

1. Was a method of randomization performed?

Yes/No/Don't know

2. Was the treatment allocation concealed?

$$
\text { Yes/No/Don't know }
$$

c. Were groups similar at baseline regarding the most important prognostic indicators?

Yes/No/Don't

know

Intervention

d. Were the index and control interventions explicitly described?

Yes/No/Don't

know

e. Was the care provider blinded to the intervention?

Yes/No/Don't know 
f. Were co-interventions avoided or comparable?

Yes/No/Don't know

g. Was the compliance acceptable in all groups?

Yes/No/Don't know

h. Was the patient blinded to the intervention?

$$
\text { Yes/No/Don't know }
$$

Outcome measurement

i. Was the outcome assessor blinded to the intervention? Yes/No/Don't know

j. Were the outcome measures relevant? Yes/No/Don't know

k. Were adverse effects described?

Yes/No/Don't know

1. Was the withdrawal/drop-out rate described and acceptable?

know

m. Timing follow-up measurements

1. Was a short-term follow-up measurement performed? Yes/No/Don't know

2. Was a long-term follow-up measurement peformed? Yes/No/Don't know

n. Was the timing of the outcome assessment in both groups comparable?

Yes/No/Don't know

\section{Statistics}

o. Was the sample size for each group described?

Yes/No/Don't know

p. Did the analysis include an intention-to-treat analysis? know

q. Were point estimates and measures of variability presented for the primary outcome measures?

Yes/No/Don't know

Yes/No/Don't 


\subsection{References}

1. Chen R, Cohen LG, Hallett M. Nervous system reorganization following injury. Neuroscience 2002;111:761-773

2. Gu Q. Neuromodulatory transmitter systems in the cortex and their role in cortical plasticity. Neuroscience 2002;111:814-835

3. Carmichael ST. Plasticity of cortical projections after stroke. Neuroscientist 2003;9:6475

4. Rossini PM, Dal Forno G. Integrated technology for evaluation of brain function and neuronal plasticity. Phys Med Rehabil Clin N Am 2004;25:263-306

5. Bütefisch CM, Netz J, Weßling M, Seitz RJ, Hömberg V. Remote changes in cortical excitability after stroke. Brain 2003;126:470-481

6. Bütefisch CM. Plasticity in the human cerebral cortex: lessons from the normal brain and from stroke. The Neuroscientist 2004;10:163-173

7. Fazelli MS, Collingridge GL. In: Cortical Plasticity LTP and LTD. BIOS Scientific Publishers Limited, 1996

8. Calautti C, Baron J. Functional neuroimaging studies of motor recovery after stroke in adults. A review. Stroke 2003;34:1553-1566

9. Martinsson L; Hårdemark H; Eksborg S. Amphetamines for improving recovery after stroke. Cochrane Database Syst Rev. 2007 Jan 24;(1):CD002090

10. Treig T, Werner C, Sachse M, Hesse S. No benefit from D-amphetamine when added to physiotherapy after stroke: a randomized, placebo-controlled study. Clin Rehabil 2003;17:590-599

11. Platz T, Kim IH, Engel U, Pinkowski C, Eickhof C, Kutzner M. Amphetamine fails to facilitate motor performance and to enhance motor recovery among stroke patients with mild arm paresis: interim analysis and termination of a double blind, randomized, placebo-controlled trial. Restor Neurol Neurosci 2005;23:271-280

12. Gladstone DJ, Danells CJ, Armesto A, Mcllroy WE, Staines WR, Graham SJ, Herrmann N, Szalai JP, Black SE. Physiotherapy coupled with dextroamphetamine for rehabilitation after hemiparetic stroke: a randomized, double-blind, placebo-controlled trial. Stroke 2006;37:179-185

13. Rösser N, Floël A. Pharmacological enhancement of motor recovery in subacute and chronic stroke. Neurorehab 2008;23:95-103 
14. Scheidtmann K, Fries W, Müller F, Koenig E. Effect of levodopa in combination with physiotherapy on functional motor recovery after stroke: a prospective, randomised, double-blind study. Lancet 2001;358:787-790

15. Sonde L, Lökk J. Effects of amphetamine and/or L-dopa and physiotherapy after stroke a blinded randomized study. Acta Neurol Scand 2007;115:55-59

16. Grade C, Redford B, Chrostowski J, Toussaint L, Blackwell B. Methylphenidate in early poststroke recovery: a double-blind, placebo-controlled study. Arch Phys Med Rehabil 1998;79:1047-1050

17. Costa RM. Plastic corticostriatal circuits for action learning: what's dopamine got to do with it? Ann N Y Acad Sci 2007;1104:172-191

18. Kandel ER, Schwartz JH, Jessell TM. In: Principles of Neural Science. Fourth edition; McGraw-Hill, 2000

19. Miyai I, Reding MJ. Effects of antidepressants on functional recovery following stroke: a double-blind study. J Neuro Rehab 1998;12:5-13

20. Dam M, Tonin P, De Boni A, Pizzolato G, Casson S, Ermani M, Freo U, Piron L, Battistin L. Effects of fluoxetine and maprotiline on functional recovery in poststroke hemiplegic patients undergoing rehabilitation therapy. Stroke 1996;27:1211-1214

21. Robinson RG, Schultz SK, Castillo C, Kopel T, Kosier JT, Newman M, Curdue K, Petracca G, Starkstein SE. Nortriptyline versus fluoxetine in the treatment of depression and in short-term recovery after stroke: a placeb-controlled, double-blind study. Am J Psychiatry 2000;157:351-359

22. Kraus RL, Li Y, Jovanovska A, Renger JJ. Trazodone inhibits T-type calcium channels. Neuropharmacology 2007; 53:308-317

23. Jacobs BL, Fornal CA. Serotonin and motor activity. Curr Opin Neurobiol 1997;7:820-825

24. Van Tulder MW, Assendelft WJJ, Koes BW, Bouter LM. Method Guidelines for systematic reviews in the Cochrane Collaboration back review group for spinal disorders. Spine $1997 ; 22: 2323-2330$

25. Pariente J, Loubinoux I, Carel C, Albucher J, Leger A, Manelfe C, Rascol O, Chollet F. Fluoxetine modulates motor performance and cerebral activation of patients recovering from stroke. Ann Neurol 2001;50:718-729

26. Zittel S, Weiller C, Liepert J. Citalopram improves dexterity in chronic stroke patients. Neurorehabil Neural Repair 2008;22:311-314 
27. Zittel S, Weiller C, Liepert J. Reboxetine improves motor function in chronic stroke. A pilot study. J Neurol 2007; 254:197-201

28. Hasbroucq T, Rihet P, Blin O, Possamai CA. Serotonin and human information processing: fluvoxamine can improve reaction time performance. Neurosci Lett 1997;229:204-208

29. Rihet P, Hasbroucq T, Blin O, Possamai CA. Serotonin and human information processing: an electromyographic study of the effects of fluvoxamine on choice reaction time. Neurosci Lett 1999;265:143-146

30. Restemeyer C, Weiller C, Liepert J. No effect of a levodopa single dose on motor performance and motor excitability in chronic stroke patients. A double-blind placebocontrolled cross-over pilot study. Restor Neurol Neurosci 2007;25:143-150

31. Berends HI, Nijlant JM, van Putten MJ, Movig KLL, IJzerman MJ. Single dose of fluoxetine increases muscle activation in chronic stroke patients. Clin Neuropharmacol 2009; 32:15

32. Loubinoux I, Boulanouar K, Ranjeva J, Carel C, Berry I, Rascol O, Celsis P, Chollet F. Cerebral functional magnetic resonance imaging activation modulated by a single dose of the monoamine neurotransmission enhancers fluoxetine and fenozolone during hand sensorimotor tasks. J Cereb Blood Flow Metab 1999;19:1365-1375

33. Loubinoux I, Pariente J, Boulanouar K, Carel C, Manelfe C, Rascol O, Celsis P, Chollet F. A single dose of the serotonin neurotransmisstion agonist paroxetine enhances motor output: double-blind, placebo-controlled, fMRI study in healthy subjects. NeuroImage 2002;15:26-36

34. Ilic TV, Korchounov A, Ziemann U. Complex modulation of human motor cortex excitability by the specific serotonin re-uptake inhibitor sertraline. Neurosci Lett 2002;319:116-120

35. Pleger B, Schwenkreis P, Grünberg C, Malin JP, Tegenthoff M. Fluoxetine facilitates usedependent excitability of human primary motor cortex. Clin Neurophysiol 2004;115:2157-2163

36. Gerdelat-Mas A, Loubinoux I, Tombare D, Rascol O, Chollet F, Simonetta-Moreau M. Chronic administration of selective serotonin reuptake inhibitor (SSRI) paroxetine modulates human motor cortex excitability in healthy subjects. NeuroImage 2005;27:314-322

37. Loubinoux I, Tombari D, Pariente J, Gerdelat-Mas A, Franceries X, Cassol E, Rascol O, Pastor J, Chollet F. Modulation of behavior and cortical motor activity in healthy subjects by a chronic administration of a serotonin enhancer. NeuroImage 2005;27:299-313 
38. Chemerinski E, Robinson RG, Kosier JT. Improved recovery in activities of daily living associated with remission of poststroke depression. Stroke 2001;32:113-117

39. Gainotti G, Antonucci G, Marra C, Paolucci S. Relation between depression after stroke, antidepressant therapy, and functional recovery. J Neurol Neurosurg Psychiatry 2001;71:258-261

40. Bilge C, Koçer E, Koçer A, Türk Börü Ü. Depression and functional outcome after stroke: the effect of antidepressant therapy on functional recovery. Eur J Phys Rehabil Med 2008;44:13-18

41. Saxena SK, Ng TP, Koh G, Yong D, Fong NP. Is improvement in impaired cognition and depressive symptoms in post-stroke patients associated with recovery in activities of daily living? Acta Neurol Scand 2007;115:339-346

42. Hackett ML, Anderson CS, House AO. Management of depression after stroke: a systematic review of pharmacological therapies. Stroke 2005;36:1098-110 



\title{
CHAPTER 3
}

\section{Single dose of fluoxetine increases muscle activation in chronic stroke patients}

\author{
Berends HI, Nijlant J, Van Putten MJAM, Movig KLL, IJzerman MJ
}

Clinical Neuropharmacology 2009 32:1-5 


\subsection{Abstract}

\section{Objectives:}

This pilot study explores the influence of a single dose of fluoxetine $(20 \mathrm{mg}$ ) on the muscle activation patterns and functional ability of the muscles in the lower part of the arm in chronic stroke patients.

Methods:

A cross-over, placebo-controlled clinical trial was conducted in 10 patients. After administration of either a single dose of fluoxetine $(20 \mathrm{mg})$ or a placebo, muscle activation of the lower arm was measured during maximum (isometric) force of the musculus flexor carpi radialis and musculus extensor carpi radialis. Delay times, grip strength and Motricity Index were measured to assess functional ability.

\section{Results:}

After fluoxetine intake, a significant increase in activation was found in both agonist and antagonist muscles the paretic arm $(\mathrm{p}<0.05)$. This increase did not influence the motor function.

\section{Conclusions:}

In this pilot study it was found that fluoxetine influences motor output in chronic stroke patients. Therefore, fluoxetine may influence motor recovery after stroke. Additional studies have to be done to explore the effects of fluoxetine on motor recovery after stroke. 


\subsection{Introduction}

Stroke is one of the major causes of death, and the main cause of disability in industrialized countries. In the United States, each year about 700,000 people experience a new or recurrent stroke. Most stroke patients suffer from hemiparesis, mainly affecting the distal parts of the body. After a stroke, muscle weakness and increased co-contraction contribute to motor impairment, including disturbed coördination ${ }^{1,2}$. In addition, an increase in movement delay times is also associated with motor impairment and physical disability ${ }^{3}$. The aim of most rehabilitation techniques for stroke patients is to decrease this motor impairment through functional training. However, because many stroke patients still suffer from physical impairments after such training, the question arises as to how these therapies can be made more effective.

A possible way to improve recovery is to stimulate processes in the brain that occur during rehabilitation. In the first days after stroke, rehabilitation presumably depends on resolution of edema and recovery of neurons in the penumbra. A few weeks after the stroke, rehabilitation probably depends on various processes commonly referred to as brain plasticity. In brain plasticity certain areas of the brain take over the functions previously performed by the damaged areas. The mechanisms that are assumed to contribute to brain plasticity include activation of previously silent synapses and pathways, sprouting, and long-term potentiation (LTP) (see for an extensive review Rossini et $\mathrm{al}^{4}$ or Chen et $\mathrm{al}{ }^{5}$ ). Function might therefore be expected to improve as the availability and excitability of corticospinal connections increase after a stroke ${ }^{6}$. The mechanisms during rehabilitation and the excitability of the corticospinal connections might also be improved by increasing the effect of certain neurotransmitters in the brain. Several studies have examined the effect of drugs which increase the amount of dopamine, norepinephrine, and/or serotonin in the brain, some of which seem to improve or accelerate motor recovery ${ }^{7-12}$. One group of drugs that probably influences this process of motor rehabilitation are the selective serotonin reuptake inhibitors (SSRIs). These drugs primarily mediate the serotonergic system by inhibiting reuptake of serotonin in the synaptic cleft, thereby increasing the amount of serotonin at the postsynaptic receptors. This increase might improve rehabilitation after stroke, because the serotonin system plays an important role in motor function ${ }^{13}$.

In healthy subjects, fluoxetine (an SSRI) was found to modulate motor performance and brain activity ${ }^{10,14-17}$. In an fMRI study, it was found that, after administration of a single dose $(20 \mathrm{mg})$, there was more focused activation in the contralateral sensorimotor area, a greater involvement of the posterior supplementary motor area, and a widespread decrease in bilateral cerebellar activation in a hand motor task ${ }^{15}$. After intake of a single dose of paroxetine (20 $\mathrm{mg}$ ) (another SSRI) a hyperactivation in the contralateral primary sensorimotor cortex and supplementary motor area, and widespread hypoactivation of the basal 
ganglia and cerebellum was found ${ }^{14}$. Long-term administration of paroxetine (20 mg daily for a period of 30 days) in healthy subjects resulted in a hypoactivation of the primary sensorimotor cortex, which correlated with improvement in the performances of a motor function test ${ }^{16}$.

At present, fluoxetine is primarily prescribed for stroke patients as an antidepressant. However, the results of some studies suggest that fluoxetine may also be beneficial in improving motor function in stroke patients. These studies found a hyperactivation in the ipsilesional primary motor cortex during an active motor task, and an improvement in the finger-tapping task in stroke patients, after intake of a single dose of fluoxetine $(20 \mathrm{mg}){ }^{10}$. During long-term administration, several weeks after the stroke occurred, fluoxetine intake $(20 \mathrm{mg}$ daily for a period of 3 months) improved motor function more than maprotiline (norepinephrine reuptake inhibitor) intake ${ }^{18}$. However, in another study no differences were found between long-term use of fluoxetine and placebo on motor function in nondepressed patients ${ }^{19}$. In healthy subjects, Pleger et al. also found no effect of fluoxetine $(20 \mathrm{mg})$ on motor performance, but they did find evidence for a usedependent facilitating effect on cortical excitability ${ }^{20}$. No explanation has yet been found for these inconsistent results.

This pilot study measured changes after the intake of a single dose of fluoxetine $(20 \mathrm{mg}$ ) in a more sensitive way. Muscle weakness, co-contraction, and abnormal delayed initiation and termination of muscle contraction may contribute to motor impairment and physical disability ${ }^{1,2,3}$. Therefore, the muscle activity and motor reaction times of chronic stroke patients were measured after the administration of a single dose of fluoxetine. As a secondary measure, motor function was assessed by measuring the grip strength and the Motricity Index (MI).

\subsection{Materials and Methods}

\section{Clinical material and study design}

Ten patients were included in this study ( 9 males, 1 female). They all had suffered an ischemic stroke, confirmed by CT-scan or MRI-scan, more than 6 months before the start of the trial. They were over 18 years of age, and were able to perform certain selective movements with the paretic wrist (Medical Research Council $(\mathrm{MRC}) \geq 2$ ).

The patients were recruited by rehabilitation physicians from two different hospitals and a rehabilitation center. Randomization and production of the placebo and the fluoxetine $(20 \mathrm{mg}$ ) was carried out by the pharmacologist in the local hospital. The research protocol was approved by the local Ethics Committee, and all patients gave written informed consent. 
The study design was a cross-over, placebo-controlled (double-blind), randomized trial. To exclude carry-over effects, the patients were measured on two different days, with an interval of 2 weeks in order to ensure an appropriate wash-out period. Half-life of a single dose of fluoxetine is 2 days, while half-life of its active metabolite norfluoxetine is 5 to 6 days $^{21}$.

All measurements were performed twice on each day, once before intake of either placebo or $20 \mathrm{mg}$ fluoxetine (baseline) and once after intake. There was an interval of 5 hours between the two measurements, in order to obtain the maximum plasma concentration of fluoxetine.

Muscle activity was recorded by surface electromyography (sEMG) ${ }^{2}$. The sample frequency was set at $2048 \mathrm{~Hz}$, the low pass filter was set at $553 \mathrm{~Hz}$ (digital FIR), and the high pass filter was set at $3 \mathrm{~Hz}$ ( $1^{\text {st }}$ order). The surface electrodes that were used were Neuroline, type $72000-\mathrm{S}(\mathrm{Ag} / \mathrm{AgCl}$, wet gel, $20 \times 30 \mathrm{~mm})$, manufactured by Ølstykke in Denmark. The electrodes were placed over the belly of the musculus extensor carpi radialis (ECR) and the musculus flexor carpi radialis (FCR) on 1/3 of the distance (proximal) on the line between the lateral, respectively medial epicondyl of the humerus and the processus styloideus of the radius. The reference electrode was placed on the processus styloideus. The electrodes were renewed for each set of measurements. Previous measurements in our lab and baseline comparements have shown sufficient reliability of this protocol (intraclass correlation $>0.90$ ).

All measurements were performed in the same way and in the same sequence. The randomization code was retained by the pharmacologist, and was only broken after the data had been processed.

\section{Measurement protocol}

When the patients visited the lab, grip strength and $\mathrm{MI}^{22,23}$ were first measured to investigate whether fluoxetine had any effect on functional motor outcome. Grip strength was measured three times by pinching a dynamometer. The mean of the three trials was calculated to obtain the most reliable estimate ${ }^{24}$. The peak grip force was also calculated.

Subsequently, muscle activity, co-contraction, and delay times were measured. After the electrodes were placed, the arm of the patient was stabilized in the apparatus (Figure 1). The position of the wrist was not forced, because the patient had to relax the arm to minimize baseline activity. After relaxation, the patient had to generate a maximum force 6 times, for 5 seconds in either the ECR (3x) or the FCR (3x). This order was randomized. A 5-second auditory stimulus was used as a trigger. The patient had to contract as quickly as possible after the start of the auditory stimulus, and when the stimulus stopped the patient had to relax the arm as quickly as possible. The EMG of both the FCR and the ECR was measured simultaneously during maximum flexion and extension contraction. 
All measurements were first performed on the non-affected side, and subsequently on the affected side.

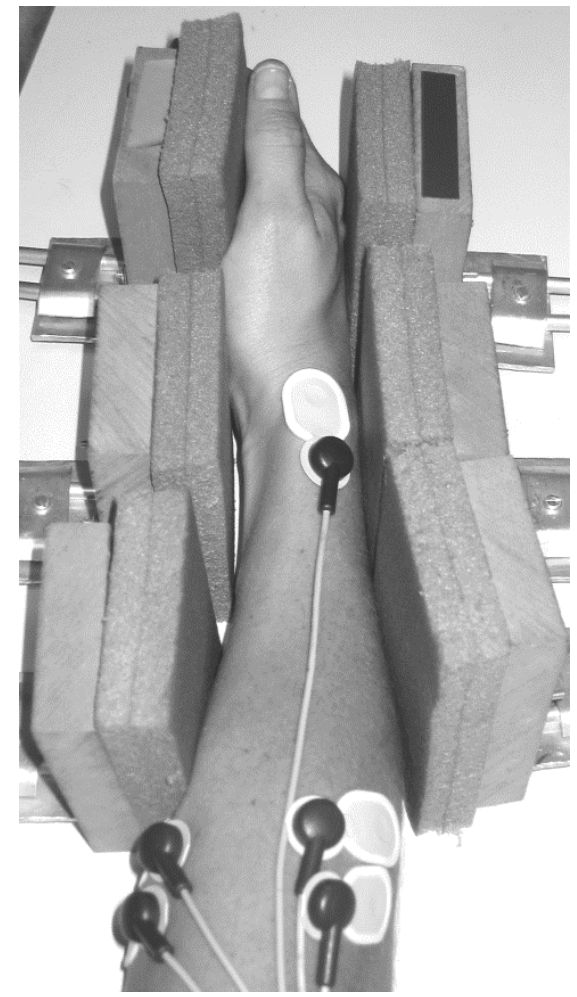

Figure 1: The arm of the patient is stabilized. Electrodes are placed on the ECR and FCR

ECR: musculus extensor carpi radialis; FCR: musculus flexor carpi radialis

\section{$\underline{\text { Analysis }}$}

Activation of the agonist and antagonist muscles was determined by the root mean square (RMS) of the EMG signal, calculated over the last 3 seconds of the stimulus. The first 2 seconds were not used, in order to exclude delay times. The RMS was calculated by $\mathrm{V}_{\mathrm{rms}}=\left[\int \mathrm{v}^{2} \mathrm{dt} / \mathrm{T}\right]^{1 / 2}$, where $\mathrm{T}=3$ (last 3 seconds of the stimulus) and $\mathrm{v}$ is the voltage of the recorded EMG signal. The RMS of both agonist and antagonist muscles was calculated.

Co-contraction was defined as ${ }^{1}: \frac{\mathrm{RMS}_{\text {antagonist }}}{\mathrm{RMS}_{\text {agonist }}}$

Delay times were calculated by the time between the onset of the beep and the change in EMG from baseline (initiation), and between the end of the auditory stimulus and "EMG back to baseline" (termination) ${ }^{3}$ (Figure 2). All calculated values were visually inspected post-hoc. 


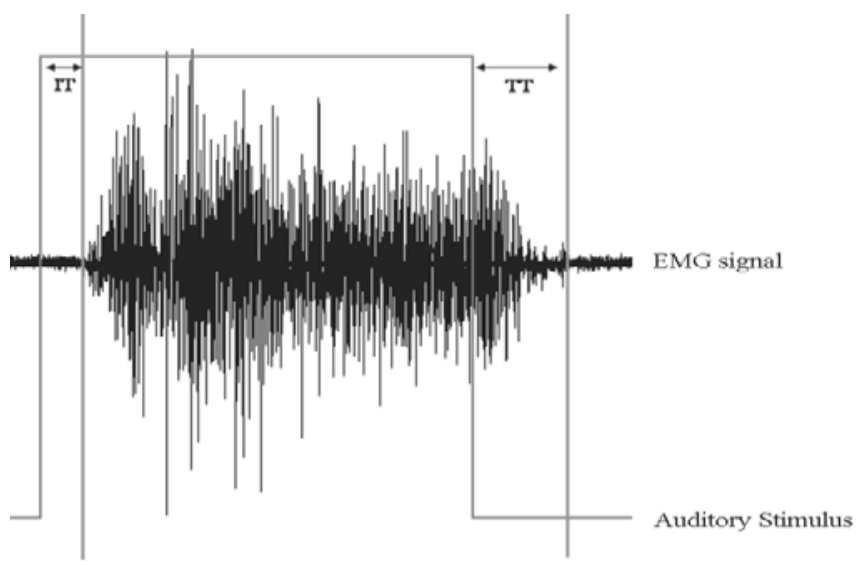

Figure 2: Electromyographic signal

IT: Initiation time

TT: termination time

To evaluate differences in muscle activation, co-contraction, delay times, mean grip strength, and MI scores, the mean of the three trials was calculated for each patient. Finally, relative differences for each patient and each intervention were calculated by normalizing the baseline values by: $\frac{X_{\text {baseline }}-X_{\text {postinterention }}}{X_{\text {baseline }}}$, where $X$ is the measured score.

Differences between placebo and fluoxetine were determined by analysis of variance (ANOVA), in which the sequence of intervention was included as a separate factor. Post-hoc analyses were Student's paired t-tests.

All statistical analyses were performed in SPSS 11.5 for Windows. $\mathrm{P}<0.05$ was considered to be significant.

\subsection{Results}

The mean time from stroke onset to study intervention was $39.1 \pm 33.6$ months, with a range of 6-120 months. All patients had a first ischemic stroke, 5 patients in the right hemisphere, and 5 in the left hemisphere. One patient had a lacunar infarction in the basal ganglia, 5 patients had an infarction in the territory of the middle cerebral artery, one patient had a carotis dissection, and one patient suffered from a lesion in the cerebellum and pons. Two patients only had a CT scan soon after the stroke occurred, in these scans the ischemic stroke was confirmed. However, the affected area was not clear. Clinically these patients seemed to have an infarction in the territory of the middle cerebral artery.

The patients were randomly divided into 2 groups: group 1 first received fluoxetine, followed by placebo ( 5 males; mean time since stroke: 48 months), and group 2 first received placebo followed by fluoxetine ( 4 males, 1 woman; mean 
time since stroke: 30.2 months). All patients were able to generate an isometric force of the wrist flexors and extensors, and had a minimum score of 2 on the MRC. None of the patients mentioned any side-effects. The results are summarized in Table 1.

Table 1: Relative differences of all measured variables

\begin{tabular}{|c|c|c|c|c|c|}
\hline \multirow[t]{2}{*}{ Variable } & \multirow[t]{2}{*}{ Arm } & \multicolumn{2}{|c|}{$\frac{X_{\text {baseline }}-X_{\text {postinterention }}}{X_{\text {baseline }}} \times 100$} & \multirow[t]{2}{*}{$\mathrm{F}$} & \multirow[t]{2}{*}{ Sign $(p)$} \\
\hline & & $\begin{array}{l}\text { Placebo } \\
(\text { mean } \pm \mathrm{sd})\end{array}$ & $\begin{array}{l}\text { Fluoxetine } \\
(\text { mean } \pm \mathrm{sd})\end{array}$ & & \\
\hline Muscle activity (RMS) & Paretic & $5.0 \% \pm 30.9 \%$ & $-21.5 \% \pm 42.7 \%$ & 10.140 & $0.002^{*}$ \\
\hline Muscle activity (RMS) & Healthy & $-1.1 \% \pm 29.2 \%$ & $-9.4 \% \pm 47.9 \%$ & 0.895 & 0.347 \\
\hline Co-contraction & Paretic & $-15.7 \% \pm 51.8 \%$ & $-14.5 \% \pm 45.9 \%$ & 0.006 & 0.939 \\
\hline Co-contraction & Healthy & $-0.77 \% \pm 39.1 \%$ & $-11 \% \pm 43.1 \%$ & 0.739 & 0.396 \\
\hline Initiation times & Paretic & $0.40 \% \pm 19.6 \%$ & $1.9 \% \pm 30 \%$ & 0.011 & 0.918 \\
\hline Initiation times & Healthy & $-10.9 \% \pm 39.3 \%$ & $8.3 \% \pm 29.5 \%$ & 2.84 & 0.105 \\
\hline Termination times & Paretic & $-7.0 \% \pm 61.6 \%$ & $-27.5 \% \pm 85.7 \%$ & 0.277 & 0.604 \\
\hline Termination times & Healthy & $-6.62 \% \pm 104 \%$ & $-13.5 \% \pm 105 \%$ & 0.040 & 0.842 \\
\hline Mean grip strength (kg) & Paretic & $-2.0 \% \pm 8.32 \%$ & $1.3 \% \pm 6.18 \%$ & 1.22 & 0.285 \\
\hline Mean grip strength $(\mathrm{kg})$ & Healthy & $-1.3 \% \pm 5.65 \%$ & $2.3 \% \pm 4.88 \%$ & 2.81 & 0.113 \\
\hline Max grip strength (kg) & Paretic & $-7.1 \% \pm 13.2 \%$ & $2.00 \% \pm 9.08 \%$ & 4.713 & $0.045^{*}$ \\
\hline Max grip strength (kg) & Healthy & $-3.43 \% \pm 8.0 \%$ & $2.4 \% \pm 5.55 \%$ & 3.872 & 0.067 \\
\hline MI & Paretic & $1.1 \% \pm 3.59 \%$ & $-2.6 \% \pm 5.72 \%$ & 3.262 & 0.090 \\
\hline
\end{tabular}

*significant: $\mathrm{p}<0.05$

RMS: root mean square

Sign: significance

Max grip strength: maximum grip strength MI: Motricity Index

\section{Muscle activity and co-contraction}

There was a mean increase of $21.5 \%$ ( $\mathrm{sd}=42.7 \%$ ) in RMS (muscle activity) in the paretic arm after intake of fluoxetine and a mean decrease of $5.0 \%$ ( $\mathrm{sd}=30.9 \%$ ) after placebo. This difference was significant $(F=10.140, p<0.002$ ) (Figure 3). The effect of fluoxetine did not differ for flexion or extension movements, or sequence of intervention, or ECR and FCR contractions. Thus, the activation of both agonist and antagonist muscles increased during flexion as well as during extension.

There was no change in co-contraction in the paretic arm, indicating that fluoxetine has no selective influence on agonist or antagonist activity. 


\section{Relative Difference RMS}

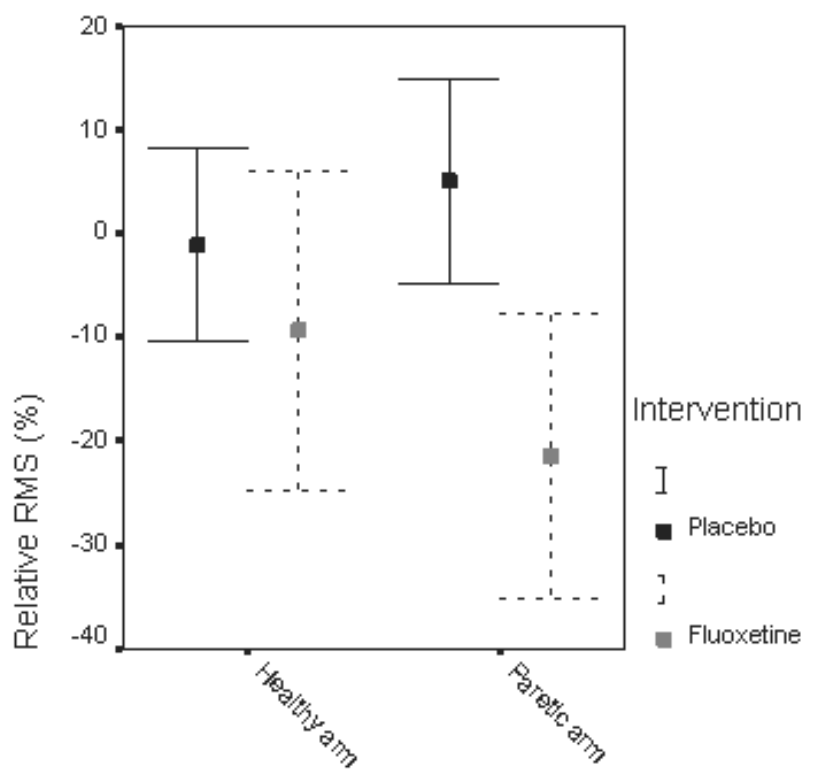

Figure 3: Mean and 95\% confidence interval

In the healthy arm, although the differences were not significant, the RMS increased after intake of fluoxetine. There was no significant change in cocontraction.

\section{Delay times}

The delay times of 7 patients were analyzed, because 1 patient had already contracted the muscles before the auditory stimulus started, and the delay times of 2 patients were not correctly registered.

After fluoxetine intake there was no change in the relative initiation and termination times in the paretic arm. In the healthy arm, the relative initiation times decreased after fluoxetine intake and increased after placebo, but these differences were not significant. There was no change in the relative termination times.

\section{Grip strength}

Table 2 summarizes the absolute mean values of the grip strength of the paretic arm.

There was no significant change in the relative mean grip strength in the paretic arm or in the healthy arm. However, the relative maximum grip strength in the paretic arm decreased significantly after intake of fluoxetine $(p<0.05)$. There were no significant changes in maximum grip strength in the healthy arm. 
Table 2: Absolute mean values of grip strength of the paretic arm

\begin{tabular}{|l|l|l|l|l|}
\hline \multirow{2}{*}{ Patient } & \multicolumn{2}{|l|}{ Fluoxetine (kg) } & \multicolumn{2}{l|}{ Placebo (kg) } \\
\cline { 2 - 5 } & Baseline & Fluoxetine & Baseline & Fluoxetine \\
\hline 1 & 15.3 & 16.7 & 17.3 & 19.3 \\
\hline 2 & 30.3 & 28.3 & 33.7 & 32.0 \\
\hline 3 & 52.0 & 53.0 & 45.7 & 48.7 \\
\hline 4 & 31.0 & 32.7 & 33.0 & 33.0 \\
\hline 5 & 14.0 & 14.3 & 12.0 & 13.0 \\
\hline 6 & 19.3 & 18.0 & 17.3 & 18.3 \\
\hline 7 & 31.7 & 33.3 & 31.0 & 29.3 \\
\hline 8 & 10.7 & 10.0 & 7.6 & 8.3 \\
\hline 9 & 23.3 & 22.7 & 24.3 & 21.7 \\
\hline 10 & 10.7 & 10.3 & 9.7 & 10.7 \\
\hline
\end{tabular}

Kg: kilograms

Motricity Index (MI)

No significant results were found in the MI.

\subsection{Discussion}

A significant increase in muscle activity was found in the paretic arm after intake of a single dose $(20 \mathrm{mg}$ ) of fluoxetine. This increase was measured in agonist as well as antagonist muscles during both flexion and extension. As no difference in cocontraction was found, the increase in activity of agonist and antagonist muscle was relatively similar. The reason for the increase in activity of both agonist and antagonist muscles, could be the widespread influence of serotonin on the brain and the spinal cord. Previous studies have shown that, after intake of a single dose of an SSRI, there is an increase in extracellular levels of serotonin in the basal ganglia (striatum ${ }^{25,26}$, nucleus accumbens ${ }^{27}$ ), thalamus, hypothalamus, substantia nigra, dorsal hippocampus, visual, auditory and parietal cortices ${ }^{28}$. The effect of serotonin in the brain and spinal cord is not selective, so therefore activity in both agonist and antagonist muscles is affected. The results are in accordance with the findings of Jacobs and Fornal, who reported an increased muscle activation in animals which correlated with the activation of serotonergic neurons ${ }^{13}$.

The influence of the increased activation of the agonist is overruled by the increased activation of the antagonist, and the net effect of increased activity will not change when motor function is measured. If activity in both muscles increases, and co-contraction does not change, motor function even can decrease due to increased rigidity.

Motor function was assessed by measuring grip strength. Although the maximum grip strength was found to decrease after intake of fluoxetine, mean grip strength 
was considered to be a more reliable measure ${ }^{24}$. The mean grip strength did not change, thus the changes in muscle activity did not seem to influence motor function. However, in this study we did not measure force and EMG simultaneously. Therefore, the relationship between grip strength and muscle activity is not very reliable. In a future study it would be of additional value to measure EMG and the force generated at the same time.

Delay times also influence motor function after stroke. Delay times were defined as the time between onset (initiation) or offset (termination) of the auditory stimulus and significant change in EMG from baseline or EMG back to baseline. There was no significant change in the delay times. The delay times include the identification of the stimulus, a sensory-motor transformation and the programming and execution of the response. Previous studies have suggested that there is an improved efficiency in cerebral motor processing after intake of an SSRI in healthy subjects $16,29,30$. All of these studies used a choice reaction time task, which include the time to decide which movement had to be made. In the present study, the patient already knew which movement had to be made. Therefore, the cerebral motor processing of the response is of less influence on the total reaction time. This could explain why, in the present study, no changes were found, while decreased choice reaction times were found in previous studies.

The changes in the paretic arm were greater than the changes in the healthy arm, which were not significant. This could indicate that the hemiparetic side is more sensitive for the influence of fluoxetine. However, because relative changes were measured, fluoxetine had to establish (absolute) greater differences in the healthy hand than in the paretic hand, because the absolute values were higher on the healthy side. However, the relative changes were more useful in the present study, because they are more closely related to motor function.

Although the present study does not provide an explanation for the inconsistent results of previous studies, this study does give a reason to assume that fluoxetine influences motor output in chronic stroke patients. However, the relationship between the administration of fluoxetine and the clinical effects in stroke patients needs to be studied further. In addition, because brain plasticity only occurs after a longer period of time, a single dose of fluoxetine cannot influence this brain plasticity. At present, the effects of long-term administration of fluoxetine on motor recovery after stroke are studied. 


\subsection{References}

1.Chae J, Yang G, Park B, et al. Muscle weakness and co-contraction in upper limb hemiparesis: relationship to motor impairment and physical disability. Neurorehabil Neural Repair 2002;16:241-248.

2.Hammond M, Fitts S, Kraft G, et al. Co-contraction in the hemiparetic forearm: quantitative EMG evaluation. Arch Phys Med Rehabil 1988;69:348-351.

3.Chae J, Yang G, Park B, et al. Delay in initiation and termination of muscle contraction, motor impairment, and physical disability in upper limb hemiparesis. Muscle \& Nerve 2002;25:568-575.

4.Rossini P, Dal Forno G. Integrated technology for evaluation of brain function and neural plasticity. Phys Med Rehabil Clin N Am 2004;15:263-306

5.Chen R, Cohen L, Hallett M. Nervous system reorganization following injury. Neurosci 2002;111:761-773

6.Talelli P, Greenwood R, Rothwell J. Arm function after stroke: Neurophysiological correlates and recovery mechanisms assessed by transcranial magnetic stimulation. Clinical Neurophysiology 2006;117:1641-1659.

7.Boyeson M, Harmon R, Jones J. Comparative effects of fluoxetine, amitryptiline and serotonin on functional motor recovery after sensorimotor cortex injury. Am J Phys Med Rehabil 1994;73:76-83.

8.Crisostomo E, Duncan P, Propst M, et al. Evidence that amphetamine with phsycial therapy promotes recovery of motor fucntion in stroke patients. Ann Neurol 1988;23:94-97.

9.Goldstein L. Potential effects of common drugs on stroke recovery. Arch Neurol 1998;55:454-456.

10.Pariente J, Loubinoux I, Carel C, et al. Fluoxetine modulates motor performance and cerebral activation of patients recovering from stroke. Ann Neurol 2001;50:718-729.

11.Sivenius J, Sarasoja T, Aaltonen $\mathrm{H}$, et al. Selegiline treatment facilitates recovery after stroke. Neurorehabil Neurol Repair 2001;15:183-190.

12.Liepert J, Bauder H, Miltner W, et al. Treatment-induced cortical reorganization after stroke in humans. Stroke 2000;31:1210-1216.

13.Jacobs B, Fornal C. Activity of serotonergic neurons in behaving animals. Neuropsychopharmacology 1999;21:9S-15S.

14.Loubinoux I, Pariente J, Boulanouar $\mathrm{K}$, et al. A single dose of the serotonin neurotransmission agonist paroxetine enhances motor output: double-blind, placebocontrolled, fMRI study in healthy subjects. NeuroImage 2002;15:26-36. 
15.Loubinoux I, Boulanouar K, Ranjeva J, et al. Cerebral functional magnetic resonance imaging activation modulated by a single dose of the monamine neurotransmission enhancers fluoxetine and fenozolone during hand sensorimotor tasks. J Cereb Blood Flow Metab 1999;19:1365-1375.

16.Loubinoux I, Tombari D, Pariente J, et al. Modulation of behavior and cortical motor activity in healthy subjects by a chronic administration of a serotonin enhancer. NeuroImage 2005;27:299-313.

17.Gerdelat-Mas A, Loubinoux I, Tombari DRO, et al. Chronic administration of selective serotonin reuptake inhibitor (SSRI) paroxetine modulates human motor cortex excitability in healthy subjects. NeuroImage 2005;27:314-322.

18.Dam M, Tonin P, De Boni A, et al. Effects of fluoxetine and maprotiline on functional recovery in poststroke hemiplegic patients undergiong rehabilitation therapy. Stroke 1996;27:1211-1214.

19.Robinson R, Schultz S, Castillo C, et al. Nortriptyline versus fluoxetine in the treatment of depression and in short-term recovery after stroke: a placebo-controlled, double-blind study. Am J Psychiatry 2000;157:351-359.

20.Pleger B, Schwenkreis P, Grünberg C, et al. Fluoxetine facilitates use-dependent excitability of human primary motor cortex. Clin Neurophysiol 2004;115:2157-2163.

21.Keller T, Cambon N, Genevray M, et al. Bioequivalence study of fluoxetine hydrochloride in healthy volunteers. Arzneimittelforschung 2005;55:491-497

22.Collin C, Wade D. Assessing motor impairment after stroke: a pilot reliability study. $J$ Neurol Neurosurg Psychiatry 1990;53:576-579.

23.Sunderland A, Tinson D, Bradley L, et al. Arm function after stroke. An evaluation of grip strength as a measure of recovery and a prognostic indicator. J Neurol Neurosurg Psychiatry 1989;52:1267-1272.

24.Mathiowetz V, Weber K, Volland G, et al. Reliability and validity of grip and pinch strength evaluation. J Hand Surg 1984;9:222-226.

25.Perry K, Fuller R. Effect of fluoxetine on serotonin and dopamine concentration in microdialysis fluid from rat striatum. Life Science 1992;50:1683-1690.

26.Rutter J, Gundlah C, Auerback S. Increase in extracellular serotonin produced by uptake inhibitors is enhanced after chronic treatment with fluoxetine. Neurosci Lett 1994;171:183-186.

27.Guan X, McBride W. Fluoxetine increases the extracellular levels of serotonin in the nucleus accumbens. Brain Res Bull 1988;21:43-46.

28.Mück-Šeler D, Jevric-Causevic A, Diksic M. Influence of fluoxetine on regional serotonin synthesis in the rat brain. J Neurochem 1996;67:2434-2442. 
Chapter 3

29.Hasbroucq T, Rihet P, Blin O, et al. Serotonin and human information processing: fluvoxamine can improve reaction time performance. Neurosci Let 1997;229:204-208

30.Rihet P, Hasbroucq T, Blin O, et al. Serotonin and human information processing : an electromyographic study of the effects of fluvoxamine on choice reaction time. Neurosci Lett 1999;265:143-146 


\section{CHAPTER 4}

\section{Relation between the effects of a single dose of fluoxetine on brain activity patterns, muscle activation and motor function in chronic stroke patients}

H.I. Berends, T. Krabben, K. Movig, M.J. Ijzerman, M.J.A.M. van Putten

Submitted to Clinical Neuropharmacology 


\subsection{Abstract}

Objective

The objective of the study was to assess the effects of a single dose of fluoxetine on muscle activity and motor function, and their relation with changes in cortical activity in chronic stroke patients.

\section{Methods}

A randomized, cross-over, placebo-controlled clinical trial was conducted in 10 chronic stroke patients. After administration of either a single dose of fluoxetine (20 mg) or a placebo, cortical activity, muscle activity and motor function were measured. Cortical activity was measured using 64-channel EEG, and event related (de-)synchronization (ERS) at C3 and C4 was calculated in the alpha and beta frequency bands. Muscle activity of the extensor and flexor carpi radialis was measured using surface EMG (sEMG) during maximal flexion and extension of the wrist. The Root Mean Square of the sEMG was calculated as measure for muscle activity. Motor function was measured using the upper extremity part of the Fugl Meyer Motor Assessment score. Differences between fluoxetine and placebo were analyzed using a paired samples t-test. The relation between cortical activity, muscle activity and motor function was analyzed using Pearson's correlation coefficient.

\section{$\underline{\text { Results }}$}

After a single dose of fluoxetine, increased agonistic muscle activity and cocontraction during both wrist flexion and extension were measured. The increases in muscle activity and co-contraction were not statistically significant ( $p>0.05)$. Correlations between motor function, muscle activity and desynchronization of the alpha and beta frequency bands were moderate to strong, but did not reach statistical significance.

\section{Conclusion}

Although trends were found in an increased agonistic muscle activation after fluoxetine, the effects were too small to proof a significant effect of a single dose of fluoxetine on cortical activity, muscle activity or motor function. 


\subsection{Introduction}

After ischemic stroke, most patients suffer from impaired motor function. Rehabilitation therapies are aimed at compensating or relearning the lost abilities (Kwakkel, 2004). After initial recovery, cortical plasticity is presumed to be important for the further improvement of motor function. In order to improve the therapies currently used for physical recovery, studies are performed investigating the facilitation of neuroplasticity using psychoactive drugs. Promising relations have been found between administration of psychoactive drugs influencing amounts of dopamine, norepinephrine, or serotonin in the brain and an improved motor recovery (Berends, 2009; Chollet, 2011). One of these drugs thought to improve the recovery of motor functions is fluoxetine.

Fluoxetine is a selective serotonin reuptake inhibitor (SSRI) and increases serotonin concentrations in the synaptic cleft between neurons in the brain. Serotonin is related to facilitation of motor output and, simultaneously to suppression of sensory information processing (Gu, 2002). It is hypothesized that serotonergic neurons support the coordination of appropriate autonomic and neuroendocrine outputs to the ongoing tonic or repetitive motor activity (Jacobs, 1999).

After intake of a single dose (Loubinoux ${ }^{a}, 1999$; Loubinoux $\left.{ }^{b}, 2002\right)$ of paroxetine and fluoxetine a more focused activation was found in the contralateral sensorimotor area, a greater involvement of the posterior supplementary motor area, and a widespread decrease of bilateral cerebellar activation in an active motor task. In addition, after intake of a single dose of sertraline (another SSRI) an increased excitability of cortico-spinal neurons and spinal motorneurons was found in healthy subjects (Ilic, 2002) without a change in motor threshold (MT).

A single dose of fluoxetine $(20 \mathrm{mg})$ causes an increased activation of the ipsilesional primary motor cortex in stroke patients performing an active motor task (finger tapping test). This augmented activation was related to improved motor skills of the affected side (Robol, 2004). Additionally, after administration of a single dose of fluoxetine (20 mg), an increased excitability of the cortex is found in studies using transcranial magnetic stimulation (Pleger, 2004; Gerdelat-Mas, 2005). Furthermore, it is thought that SSRIs facilitate the activity of interneurons in the primary motor cortex (Rutter, 1994). In contrast, long term administration of serotonergic drugs was found to decrease the activation and excitability of the primary motor cortex (Gerdelat-Mas, 2005; Acler, 2009). This decreased excitability was related to an improved neurological state.

Next to the effects on brain activity, a single dose of $20 \mathrm{mg}$ fluoxetine was found to increase muscle activation on the affected side in chronic stroke patients (Berends 2009a). Muscle weakness and degree of co-contraction are found to correlate with motor impairments and physical disability after stroke (Hammond, 1988; Chae, 
2002). Hypothetically, increased muscle activity of agonist muscles after fluoxetine intake possibly improves motor function.

Long term use of fluoxetine is found to improve recovery of motor function after stroke. Miyai et al found a significantly greater improvement in total FIM (functional independence measure) scores after fluoxetine compared to stroke patients treated with desipramine (100mg) (Miyai, 1998). Dam et al found a greater improvement of motor function after intake of fluoxetine compared to maprotiline (Dam, 1996). Another study examining the effects of an SSRI (citalopram) found an improved post stroke dexterity (Zittel, 2008). Chollet et al published the first large double-blind randomized trial $(n=118)$ to examine the effects of long term administration of fluoxetine in stroke patients, shortly after stroke. They found an improved motor function after 90 days of treatment (Chollet, 2011).

The contradicting results of these studies make the effects of fluoxetine to be ambiguous. To better understand the effects of fluoxetine, the influence of a single dose of fluoxetine $(20 \mathrm{mg})$ on muscle activation and on motor function are examined. In another study we examined the effects of fluoxetine on cortical activity during movement execution in the very same patients (Berends, submitted). These results are now related to changes in muscle activity and motor function measured in the present study.

\subsection{Methods}

$\underline{\text { Subjects }}$

Included patients had to be at least 18 years old, and suffering from a single, unilateral stroke. Time post stroke had to be over 6 months (chronic stroke). Patients had to have an affected hand and/or arm function, with some voluntary muscle contraction. Exclusion criteria were other (pre-existing) neurological diseases (e.g. epilepsy, tumor, paralysis), known allergy to SSRIs, use of antidepressants, autism spectrum disorders, pervasive development disorder, schizophrenia (or history thereof), unstable medical health situation, uncompensated hemineglect or cognitive disabilities resulting in misunderstanding or incapability of the execution of instructions given, uncorrected visual problems, pregnancy, severe uncontrolled medical conditions, known alcoholism or drug abuse, and known elevated intracranial pressure.

From 2009 until 2010 eleven stroke patients (mean age: $58.5 \pm 11.16,3$ female) were included. One patient was excluded from further analysis since measurements were discontinued after notification of a previous seizure. Seven patients suffered from a left-hemispheric stroke. All patients were right handed before stroke. 
All patients gave written informed consent. The trial was approved by the institutional ethics committee and was performed in accordance with the Declaration of Helsinki. The EudraCT number is 2008-003349-97.

\section{Study design}

The study was designed as a randomized, double-blind, placebo-controlled, crossover trial.

Patients were randomly assigned to crossover treatment: they were assigned to fluoxetine $20 \mathrm{mg}$ and placebo, or vice versa. Fluoxetine was dispensed as a capsule. The corresponding placebo capsule was identical except for the fluoxetine content. The trial randomization was performed by an independent statistician. The study medication was produced and labeled before the start of the trial by an independent hospital pharmacist not otherwise involved in the trial. Patients, doctors, and researchers were unaware of treatment assignment until all patients had completed the study, and all data were analyzed.

\section{$\underline{\text { Procedure }}$}

The study consisted of 4 similar measurements divided over 2 days with 14 days in between to exclude carry-over effects. On both days, measurements were performed before intake of placebo or $20 \mathrm{mg}$ fluoxetine (baseline) and 5 hours after intake, when plasma concentration of fluoxetine peaked. Measurements were performed in the same way and followed the same sequence. Each measurement started with the assessment of motor function by using the "upper extremity part" of the Fugl-Meyer motor assessment (max. 66 points). Subsequently, muscle activity during flexion and extension of the wrist was recorded by means of surface electromyography (sEMG).

In the second (afternoon) session on both days brain activity was measured using 64-channel electroencephalography (EEG). Recordings were made in rest with eyes closed ( 1 minute) and eyes opened ( 1 minute). Thereafter, EEG was measured while the patient moved the top of the thumb to the top of the index finger (pincer grip) for $10 \times 5$ seconds, alternating both hands, and with a rest of 5 seconds in between. The EEG was only measured during the afternoon measurements, since it was too strenuous for patients to place an EEG-cap twice a day.

\section{EMG analysis}

EMG was recorded with a sample rate (FS) of $2048 \mathrm{~Hz}$. The high pass filter was set at $3 \mathrm{~Hz}$ (digital FIR) and the low pass filter was $553 \mathrm{~Hz}$ ( $1^{\text {st }}$ order). Surface electrodes (Ag/AgCl, Neuroline Ølstykke, Denmark) were placed over the muscle belly of the musculus extensor carpi radialis (ECR) and the musculus flexor carpi radialis (FCR) on $1 / 3$ of the distance (proximal) on the line originating from the 
lateral, respectively medial epicondyl of the humerus, towards the processus styloideus of the radius. The reference electrode was placed on the processus styloideus. During baseline measurements, the arm of the patient was stabilized while the position of the wrist remained neutral, allowing the patient to relax his/her arm to minimize muscle activity. After relaxation the patients had to generate a maximum voluntary contraction in either the ECR (3x) or the FCR (3x) during a 5 second auditory stimulus. The order of flexion and extension was randomized. All measurements were first performed on the non-affected side and subsequently on the affected side.

Muscle activation was determined by the root mean square (RMS) of the EMG signal, calculated over the last 3 seconds of the stimulus. The first 2 seconds were not used to exclude delay times. RMS was defined as

$R M S=\sqrt{\frac{1}{(N-k)} \sum_{k=1}^{N}\left[V_{k}\right]^{2}} k=1,2, \ldots, N$
$\mathrm{~V}_{\mathrm{rms}}=\left[\int \mathrm{v}^{2} \mathrm{dt} / \mathrm{T}\right]^{1 / 2}$

where $\mathrm{N}=5^{*} \mathrm{Fs}$ and $\mathrm{k}=2 * \mathrm{Fs}$ and $\mathrm{V}$ is the voltage of the recorded EMG signal. The average RMS of three trials was calculated for each patient.

Subsequently, relative differences for each patient $\left(\mathrm{RMS}_{\text {norm }}\right)$ were calculated by normalizing the baseline, using

$\mathrm{RMS}_{\text {norm }}=\frac{\mathrm{RMS}_{\text {baseline }}-\mathrm{RMS}_{\text {postinterention }}}{\mathrm{RMS}_{\text {baseline }}}$

Co-contraction was defined by dividing the RMS of the antagonist by the RMS of the agonist.

\section{EEG analysis}

EEG was recorded against a virtual common reference using a 64-channel Refa amplifier (ANT Neuro, TMSi, The Netherlands) using the international 10/20 system for electrode placement. The impedance of all EEG-electrodes was kept below $5 \mathrm{kOhm}$ to reduce polarization effects. Sampling frequency was $512 \mathrm{~Hz}$. EOG of the right eye was measured to monitor eye movements. Event related (de)synchronization (ERS) was defined as an increased (event related synchronization) or decreased (event related desynchronization) instant power during execution compared to rest. To analyze the ERS during execution of the pincer grip the EEG was selected while EMG was recorded over the left and right musculus abductor pollicis brevis (APB) and the right FCR. Since the patients were hardly able to relax their muscles after the execution of the movement, the signal power of the frequency bands during a 1 minute rest with eyes opened was used as 
a "rest-condition". These data were visually inspected for EMG activity. EEG data with simultaneous EMG activity has been rejected.

The EEG was offline filtered between 1 and $100 \mathrm{~Hz}$ using a $2^{\text {nd }}$ order Butterworth filter (zero-phase shift). To calculate the power changes, the EEG was filtered into 3 frequency bands (theta: 4-8 Hz, alpha: 8-13 Hz, beta: $13-25 \mathrm{~Hz}$ ) with $256^{\text {nd }}$ order zero phase forward and reverse FIR filters.

Subsequently, the average power (P) was calculated as:

$$
P=\frac{1}{N} \sum_{i=1}^{i=n} x^{2} f(i, j)
$$

where $x_{f(i, j)}$ is the $\mathrm{j}$-th sample of the $\mathrm{i}$-th trial of the bandpass filtered data.

Subsequently, the ERS is calculated as:

$E R S=\frac{P_{\text {activity }}-P_{\text {rest }}}{P_{\text {activity }}+P_{\text {rest }}}$.

An ERS value < 0 (Pactivity < Prest) represents a desynchronization of the frequency band during the execution of the movement compared to baseline.

\section{Statistical analysis}

Data analysis was performed offline. All routines were implemented in Matlab 2014b (The Mathworks, Inc, Natick, MA).

To control for differences between days, the FM scores of the baseline measurements on both days were tested using a paired samples t-test.

Differences between placebo and fluoxetine on FM scores, $\mathrm{RMS}_{\text {norm }}$ and ERS were analyzed using paired t-tests.

Correlations were analyzed using the Pearson correlations coefficients. The $\mathrm{RMS}_{\text {norm }}$ of the antagonistic and the agonistic muscle during flexion and extension was used and related to the ERS of the alpha and beta frequency bands in the ipsiand contralateral hemisphere during movement and to the FM scores of the afternoon measurements.

All statistical analyses were performed in SPSS 21 for Windows. $\mathrm{P}<0.05$ was considered to be statistically significant.

The results are checked for non-significant trends by reporting mean values of all measurements. 


\subsection{Results}

\section{Motor function}

Fugl-Meyer motor assessment (FM) scores did not differ between days or between pre- or post-measurements on one day (table 1 ).

Table 1: mean, minimum and maximum FM-scores for all 4 measurements.

\begin{tabular}{|l|l|l|l|l|}
\hline & Pre-placebo & Post-placebo & Pre-fluoxetine & $\begin{array}{l}\text { Post- } \\
\text { fluoxetine }\end{array}$ \\
\hline Mean \pm SD & $41.1 \pm 18.7$ & $43.7 \pm 18.4$ & $42.7 \pm 19.0$ & $42.7 \pm 19.5$ \\
\hline Minimum & 12 & 12 & 14 & 13 \\
\hline Maximum & 59 & 61 & 61 & 63 \\
\hline
\end{tabular}

\section{Muscle activation}

No significant differences were found between the $\mathrm{RMS}_{\text {norm }}$ after fluoxetine and after placebo for agonistic and antagonistic activity during flexion and extension of the wrist.

Table 2 summarizes the RMS of the agonistic and antagonistic muscles 5 hours after intake of the capsule.

Table 2 Mean RMS of the agonistic and antagonistic muscles during flexion and extension after intake of the capsule.

\begin{tabular}{|l|l|l|l|l|}
\hline & $\begin{array}{l}\text { Mean RMS } \\
\text { Placebo }\end{array}$ & $\begin{array}{l}\text { Mean RMS } \\
\text { fluoxetine }\end{array}$ & $\begin{array}{l}\text { Co- } \\
\text { contraction } \\
\text { placebo }\end{array}$ & $\begin{array}{l}\text { Co- } \\
\text { contraction } \\
\text { fluoxetine }\end{array}$ \\
\hline $\begin{array}{l}\text { Affected } \\
\text { Flexion agonist }\end{array}$ & $88.0 \pm 84.0$ & $97.0 \pm 107.1$ & $0.28 \pm 0.14$ & $0.39 \pm 0.47$ \\
\hline $\begin{array}{l}\text { Non-affected } \\
\text { Flexion agonist }\end{array}$ & $228.5 \pm 124.1$ & $207,5 \pm 109.6$ & $0.20 \pm 0.11$ & $0.19 \pm 0.10$ \\
\hline $\begin{array}{l}\text { Affected flexion } \\
\text { Antagonist }\end{array}$ & $17.9 \pm 10.8$ & $16.9 \pm 12.3$ & & \\
\hline $\begin{array}{l}\text { Non-affected } \\
\text { flexion antagonist }\end{array}$ & $39.1 \pm 29.6$ & $36.6 \pm 24.4$ & & $0.71 \pm 1.1$ \\
\hline $\begin{array}{l}\text { Affected extension } \\
\text { antagonist }\end{array}$ & $24.9 \pm 19.2$ & $27.5 \pm 23.8$ & $0.51 \pm 0.40$ & $0.11 \pm 0.035$ \\
\hline $\begin{array}{l}\text { Non-affected } \\
\text { extension } \\
\text { antagonist }\end{array}$ & $25.2 \pm 12.8$ & $23.3 \pm 11.6$ & $0.12 \pm 0.066$ & \\
\hline $\begin{array}{l}\text { Affected extension } \\
\text { agonist }\end{array}$ & $64.2 \pm 51.4$ & $96.4 \pm 56.0$ & & \\
\hline $\begin{array}{l}\text { Non-affected } \\
\text { extension agonist }\end{array}$ & $255.9 \pm 166.2$ & $221.7 \pm 98.3$ & & \\
\hline
\end{tabular}

The differences between fluoxetine and placebo are relatively small and the standard deviation is high because of large interindividual differences. Differences 
are found between the RMS of the affected and the non-affected arm in both agonistic and antagonistic muscles, where the RMS of both agonist and antagonist is higher in the non-affected arm during flexion. During extension, the RMS of the antagonist (flexor) is lower in the non-affected arm compared to the affected arm, while the RMS of the agonist is higher. Co-contraction was always lower in the nonaffected arm with the largest differences during extension of the wrist. In addition, the agonistic muscle activity is higher after fluoxetine during both flexion and extension.

\section{Brain activity}

The effects of fluoxetine on the ERS during movement of the hand were described in our study examining the effects of fluoxetine on cortical activity during execution and imagination (submitted). This study described an increased desynchronization of the frequency bands over the ipsilateral motor cortex (C3/C4) during movement of the healthy hand after intake of fluoxetine. No other significant differences were found.

Figure 1 shows the topoplots of the ERS of the theta, alpha and beta frequency bands of the 7 patients suffering from a left hemispheric stroke $(n=7)$. All frequency bands desynchronized during execution compared to the resting state over almost the whole cortex. The desynchronization over the motor cortex (C1/C2/C3/C4) and the somatosensory cortex (CP1/CP2/CP3/CP4) were found to be larger compared to other cortical areas.

After fluoxetine, during the execution of the movement by the affected hand, the non-affected (ipsilateral) hemisphere showed a non-significantly larger desynchronization of the theta and the beta frequency bands at C4 compared to the affected hemisphere at C3. After placebo, the beta frequency band seems to be more equally distributed between the left and right hemisphere. Desynchronization of the alpha frequency band seemed to be equally distributed between the ipsi- and the contralateral hemisphere.

After placebo, during the execution of the movement by the non-affected hand, the alpha and beta frequency bands showed the largest desynchronization over the contralateral non-affected hemisphere (C4). After fluoxetine, the desynchronization of the alpha frequency band was bilaterally equally distributed. Concurrently, after fluoxetine, a higher desynchronization of the theta frequency band was found in the ipsilateral, affected hemisphere. 
Correlations of cortical activity, muscle activation and motor function After placebo, correlations between ERS of the alpha and beta frequency bands and RMS of the agonistic and antagonistic muscles during flexion and extension of the affected wrist were between 0.011 and 0.499 considering the ipsilateral hemisphere, and between -0.006 and 0.233 at the contralateral hemisphere. None of these correlations were statistically significant.

After fluoxetine, correlations between ERS of the alpha and beta frequency bands and RMS of the agonistic and antagonistic muscles during flexion and extension of the affected wrist were between -0.371 and 0.342 considering the ipsilateral hemisphere, and between -0.388 and 0.620 at the contralateral hemisphere. Although the correlations were not statistically significant, a correlation of 0.620 is found to be strong (Cohen, 1988).

No differences were found in the FM scores after fluoxetine, and only moderate to weak non-significant correlations were found between FM and ERS, or RMS (range between $r=-0.404$ and $r=0.348$ ). 
Movement affected (right) hand

Movement nonaffected (left) hand

\section{Fluoxetine Frequency band Placebo}

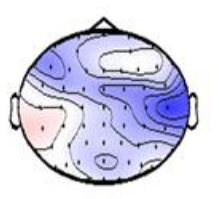

Theta
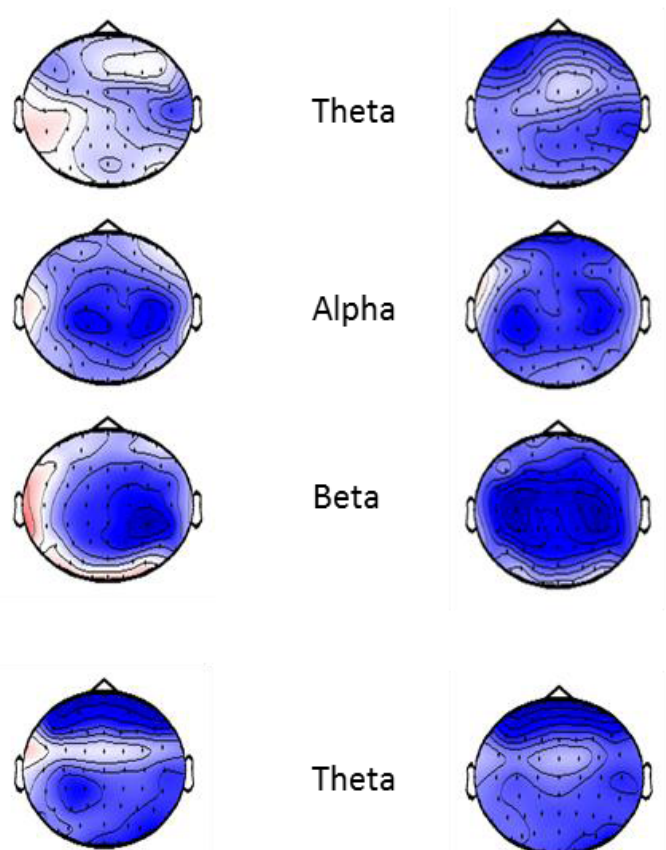

Alpha

Beta

Theta
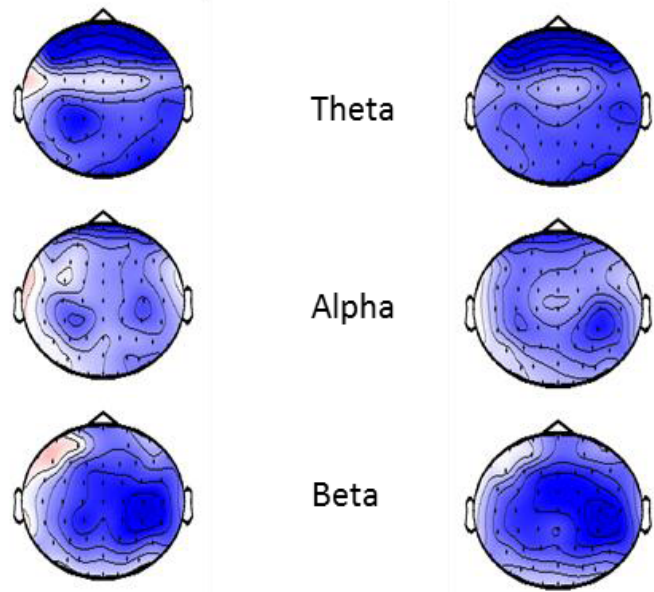

Figure 1 Topoplots of the event related synchronization in 7 left hemispheric stroke patients. The left column is the ERS after fluoxetine, and the right column after placebo. The negative values (blue colour) indicate a decreased power of the frequency band during the movement compared to rest.

\subsection{Discussion}

This study examines the effects of a single dose of fluoxetine on the muscle activity and brain activity in chronic stroke patients. Although no statistically significant results were found for fluoxetine on motor function, on muscle activity, or on cortical activity of the motor cortex, trends were found. Correlations between muscle activity and the desynchronization of the alpha and beta frequency bands 
during movement execution were strong, moderate to weak (Cohen, 2011), but did not reach statistical significance.

A further contemplation of the results in the present study confirms the results of previous studies. The muscle activity of the non-affected arm was higher compared to the affected arm, while the co-contraction of the affected arm was higher. Previous studies found an increased muscle weakness and co-contraction in the affected arm after stroke (Hammond 1988, Chae 2002). For the effect of fluoxetine a trend was found in increased agonistic muscle activity and co-contraction. These results agree with the results of our previous study. Although an increased muscle activity was previously found to improve motor function (Hammond, 1988; Chae, 2002), the increased co-contraction might nullify this effect.

Previously published results examining the effects of psychoactive drugs on the rehabilitation after stroke did show significant results. An effect of fluoxetine was previously found on motor function (Dam, 1996; Miyai, 1998; Pariente, 2001), the power of EMG (Berends, 2009a), and on cortical activity (Gerdelat-Mas, 2005). However, other studies examining the potential of these kinds of drugs to improve the rehabilitation of motor function did not found any effects (Restemeyer, 2007; Berends, 2009a). The present study did find a relation between the muscle activity of the affected hand and the cortical activity over the contralateral motor cortex. A trend was found in an increased agonistic muscle activity after fluoxetine; when this relates to the cortical activity a positive trend in increased cortical activity can be expected. Although this latest suggestion cannot be confirmed by visual inspection of the topoplots, our previous study describes a significant higher desynchronization of the alpha frequency band in the affected hemisphere during movement of the healthy hand (Berends, submitted).

Both desynchronization during imagination of movements and muscle activity have large within- and between-subject variabilities (Berends, 2009; Tangwiriyasakul, 2014), and one should be careful drawing conclusions. This high variability might blur the results, especially when these two different kinds of measurements (cortical activity and muscle activity) are to be correlated. This high variability might be due to other confounders influencing the results. The group "stroke patients" is a heterogeneous group as patients suffer from different impairments caused by different dimensions and sites of stroke. Next to the impaired motor function, other abilities might also be affected, for instance cognitive functioning or sensory functioning. Fluoxetine has a widespread effect in the brain including the areas involved in cognitive functioning. Therefore, the effect of the drug on the rehabilitation of motor function might also be caused by an indirect effect on for instance corticocortical connections, sensorimotor connections, or activity in the prefrontal areas. These confounders might also explain the contradicting results of previous studies. To clarify the differences 
between and within studies, future studies should focus on the differences between responders and non-responders to the treatment and should more emphasize the not-significant results.

In conclusion, trends were found in an increased muscle activity and cocontraction despite many confounders affecting the results. No statistically significant relations between cortical activity, muscle activity and motor function were found. 


\subsection{References}

Acler M, Robol E, Fiaschi A, Manganotti P. A double blind placebo RCT to investigate the effects of serotonergic modulation on brain excitability and motor recovery in stroke patients. J Neurol 2009 : 256 1152-1158

Berends HI, Nijlant J, Movig KLL, Van Putten MJAM, Jannink MJA, IJzerman MJ. 2009. The clinical use of drugs influencing neurotransmitters in the brain to promote motor recovery after stroke: a Cochrane systematic review. Eur J Phys Rehabil Med. 45: 621-630

Berends HI, Nijlant J, Van Putten M, Movig KLL, IJzerman MJ. Single dose of fluoxetine increases muscle activation in chronic stroke patients. Clin Neuropharmaco 2009a;32:1-5

Chae J, Yang G, Park B, Labatia I. Muscle weakness and co-contraction in upper limb hemiparesis: relationship to motor impairment and physical disability. Neurorehabil Neural Repair. 2002;16:241-248.

Chollet F; Tardy J, Albucher JF, et al. Fluoxetine for motor recovery after acute ischaemic stroke (FLAME): a randomised placeb-controlled trial. Lancet Neurol 2011: 10(2): 123-130.

Cohen J. Statistical power analysis for the behavioral sciences. 1988. New Jersey Lawrence Erlbaum

Dam M, Tonin PDBA, Pizzolato G, et al. Effects of fluoxetine and maprotiline on functional recovery in poststroke hemiplegic patients undergoing rehabilitation therapy. Stroke 1996;27:1211-1214.

Gerdelat-Mas A, Loubinoux I, Tombari D, Rascol O, Chollet F, Simonetta-Moreau M. Chronic administration of selelctive serotonin reuptake inhibitor (SSRI) paroxetine modulates human motor cortex excitability in healthy subjects. NeuroImage 2005;27:314-322

$\mathrm{Gu}$ Q. Neuromodulatory transmitter systems in the cortex and their role in cortical plasticity. Neuroscience 2002;111:814-835

Hammond M, Fitts S, Kraft G, Nutter P, Trotter M, Robinson L. Co-contraction in the hemiparetic forearm: quantitative EMG evaluation. Arch Phys Med Rehabil. 1988;69:348351.

Ilic T, Korchounov A, Ziemann U. Complex modulation of human motor cortex excitability by the specific serotonin re-uptake inhibitor sertraline. Neuroscience Letters 2002;319:116120.

Jacobs B, Fornal C. Activity of serotonergic neurons in behaving animals. Neuropsychopharmacology 1999;21:9S-15S

Kwakkel G, Kollen B, Lindeman E. Understanding the pattern of functional recovery after stroke: facts and theories. Restor Neurol Neurosci 200422 281-299 
Loubinouxa I, Boulanouar K, Ranjeva J, Carel C, Berry I, Rascol O. Cerebral functional magnetic resonance imaging activation modulated by a single dose of the monomine neurotransmission enhancers fluoxetine and fenozolone during hand sensorimotor tasks. Journal of Cerebral Blood Flow and Metabolism 1999;19:1365-1375.

Loubinoux ${ }^{b}$ I, Pariente J, Boulanouar K, et al. A Single Dose of the Serotonin Neurotransmission Agonist Paroxetine Enhances Motor Output: Double-Blind, PlaceboControlled, fMRI Study in Healthy Subjects. NeuroImage 2002;15:26-36.

Miyai I, Reding M. Effects of antidepressants on functional recovery following stroke: a double-blind study. J Neuro Rehab 1998;12:5-13.

Pleger B, Schwenkreis P, Grünberg C, Malin J-P, Tegenthoff M. Fluoxetine facilitates usedependent excitability of human primary motor cortex. Clinical Neurophysiology 2004;115:2157-2163.

Restemeyer C, Weiller C, Liepert J. No effect of a levodopa single dose on motor performance and motor excitability in chronic stroke patients. A double-blind placebo-controlled crossover pilot study. Restor Neurol Neurosci 2007;25:143-150

Robol E, Fiaschi A, Manganotti P. Effects of citalopram on the excitability of the human motor cortex: a paired magnetic stimulation study. Journal of the Neurological Sciences 2004;221:41-46.

Rutter J, Gundlah C, Auerback B. Increase in extracellular serotonin produces by uptake inhibitors is enhanced after chronic treatment with fluoxetine. 1994 Neuro sci Lett 171: $183-186$

Tangwiriyasakul C Verhagen R, Van Putten MJAM, Rutten WLC. Importance of baseline in event-related desynchronization during a combination task of motor imagery and motor observation. 2013. Journal of neural engineering 10:026009

Zittel S, Weiller C, Liepert J. Citalopram improves dexterity in chronic stroke patients. Neurorehabil Neuro Repair 2008. 22: 311-312 



\section{CHAPTER 5}

\section{Differential cortical activation during observation and observation-and-imagination}

Berends HI, Wolkorte R, IJzerman MJ, Van Putten MJAM

Exp Brain Res 2013: 229:337-345 


\subsection{Abstract}

Objective

The activity of the brain during observation or imagination of movements might facilitate the relearning of motor functions after stroke. The present study examines if there is an additional effect of imagination over observation-only.

\section{Methods}

Eight healthy subjects observed and observed-and-imagined a movement of a hand. 64-Channel EEG was used to measure brain activity. The synchronization of the theta $(4-8 \mathrm{~Hz})$, alpha $(8-13 \mathrm{~Hz})$ and beta $(13-25 \mathrm{~Hz})$ frequency bands was calculated, and plotted in topoplots. The temporal changes of the sensorimotor area $(\mathrm{C} 3, \mathrm{C} 4)$ and the centro-parietal cortex $(\mathrm{Pz})$ were analyzed in the two experimental conditions.

\section{$\underline{\text { Results }}$}

During observation-and-imagination a significant larger desynchronization $(p=0.004)$ in the sensorimotor area was found compared to observation-only in all electrodes and frequency bands. In addition, temporal differences were found between observation and observation-and-imagination in the alpha frequency bands.

\section{Conclusions}

During observation-and-imagination, modulations of EEG rhythms were stronger than during observation-only in the theta, alpha and beta frequency bands and during almost the whole activity fragment. These findings suggest an additive effect of imagination to observation in the rehabilitation after stroke.

Keywords: Mirror Neuron, Imagination, Observation, EEG, Event related Synchronization 


\subsection{Introduction}

Neurophysiological experiments in animals and humans have shown that similar brain areas are activated during execution and observation of a movement. These experiments point to "a functional equivalence" or "shared motor representations" between the cortical processes underlying movement observation and execution (Babiloni et al 2001), and are thought to represent the "observation-execution matching system". These "mirror-like" properties of the brain are found during both observation and imagination of movements (Calmels et al, 2006). However, these concepts concern different tasks. Movement observation is defined as the perception of the actions of others. Motor imagery can be defined as "the covert cognitive process of imagining a movement of your own body(-part) without actually moving that body(-part)" (De Vries et al, 2007).

The activity of the brain during observation and imagination is thought to be important for the development of language (Rizzolatti et al 2004), the understanding of mimics and empathy during social interaction (Schulte-Rüther et al 2007), and in the (re-)learning of motor function. This latter function is probably relevant in neurological patients, where movement observation or imagination is found to improve the relearning of motor skills (Dijkerman et al 2004, Ertelt et al 2007, Page et al 2007).

Several studies in healthy subjects explored differences between imagination and observation. In an fMRI study, the differences between imagination and observation were studied in healthy subjects by analyzing the blood-oxygenationlevel-dependent (BOLD) activations (Filimon et al 2007). During both observation and imagination of a reach movement of the right arm, activations of the intraparietal sulcus, the superior parietal cortex, the precuneus and the dorsal premotor cortex were found compared to a baseline condition. These activations during both tasks were stronger in the left hemisphere. During imagination, the inferior parietal lobule, the posterior end of the Sylvian fissure, the supplementary motor cortex and the inferior frontal gyrus were activated, as well. Grafton et al (1996) examined the differences between observation and imagination using PETscans. They also found an activation of the dorsal premotor cortex during both tasks. In addition, they found the inferior frontal cortex, the inferior parietal cortex and the supplementary motor area to be active during both tasks. During observation, the rostral superior temporal sulcus was activated, while during imagination the middle frontal cortex was active. In accordance with the study of Filimon et al (2007), most activation was measured in the left hemisphere. Grafton et al (1996) found that movement observation and movement imagination almost coincide, except for the fact that the extent and intensity of activation were stronger during imagined grasping. (Grafton et al, 1996). Methodological differences between these studies, like different movements to be imagined, or 
different imaging techniques to be used might explain why Grafton found more areas to be activated.

When the corticospinal excitability is compared between observation, imagination and imitation, imitation was found to induce a larger increase of the corticospinal excitability compared to imagery or "passive observation" (Léonard, 2007). Roosink et al (2010) found an increased corticospinal excitability during active observation (observation with intention to imitate) compared to passive observation (without prior instructions). In patients suffering from a motor conversion disorder, a difference between the corticospinal excitability during movement observation and movement imagination has been reported too. The corticospinal motor excitability was increased during movement observation, similar to healthy subjects, while the excitability was decreased during imagination of own body movements (Liepert, 2011).

Several EEG-studies examined the effects of observation or imagination on changes in the power of predefined frequency bands. In the EEG, the $\mu$-rhythm (between 8 and $15 \mathrm{~Hz}$ ) is generated by the sensorimotor cortex and is most prominent during rest, while it is suppressed during active movements. Similar to the fMRI and PET studies, during observation and imagination of finger movements the activity in the premotor and primary sensorimotor cortex was found to modulate (Pfurtscheller et al 1999, Cochin et al 1999, Babiloni et al 2002). During observation of finger movements, a significant decrease was found in spectral power in the low-alpha frequency band (7.5-10 Hz), compared to rest. During imagination of a wrist extension, the rhythms in the alpha frequency band attenuated over the sensorimotor cortex. This event-related desynchronization (ERD) appears similar to the ERD found during the execution of the movement. However, the ERD-amplitude was larger during the execution (Bai et al 2008).

In addition to the attenuation of the alpha frequency band during the execution of a voluntary movement, $\beta$-desynchronization $(15-25 \mathrm{~Hz})$ over the Rolandic region can be observed. In a study of Babiloni et al (2002) ten healthy subjects had to execute an aimless movement of the middle finger, whereafter they had to look at another person executing the same finger movement. This study found a decreased power in the beta frequency-band during movement execution and movement observation, while the power of this frequency band increased during the postmovement period. Another study examined the behavior of the beta-frequency during imagination of wrist extension. A desynchronization of the beta-frequency (20-24 Hz) was found on the contralateral left hemisphere over the sensorimotor cortex and the supplementary motor area (Bai et al 2008). Desynchronization becomes bilaterally symmetrical just before the execution of the movement, indicating a role of these regions in the planning of the movement. Although no planning is involved in movement observation, desynchronization of the betafrequency during the observation of a movement has been reported. 
The effects of the addition of imagining or observation of a movement to the rehabilitation therapy of motor function after stroke has been examined in several studies. Ertelt et al (2007) studied the effects of action observation on rehabilitation of motor deficits after stroke. They found "pieces of evidence that action observation has a positive additional impact on recovery of motor function after stroke by reactivation of motor areas, which contain the action observation/action execution matching system" of the brain. Page et al (2007) also found support for the efficacy of incorporating mental practice of specific arm movements for rehabilitation of motor function in patients with chronic stroke, when compared to a placebo condition. Schuster et al (2012) also demonstrated an additional effect of "motor imagery" on the recovery of motor function after stroke when measuring the motor function. These results suggest an active role of observation and imagination for the rehabilitation after stroke. Therefore, theory as well as intervention studies confirm the possibility of recovery of motor function by imagination or observational training.

Since observation and imagination concern different tasks, the question remains which of both tasks is best to be used in the rehabilitation after stroke. Therefore, the present study examines if there is an additional effect of imagination over observation-only. In order to better understand these potential additional effects, we study if temporal and spatial differences are present using high density electroencephalography (EEG).

\subsection{Methods}

\section{Subjects}

Eight healthy, right-handed subjects (3 male, mean age 27.1 years) participated in the study.

\section{Experimental protocol}

Subjects watched a movie consisting of 64 fragments showing intermittently an aimless hand movement and a baseline condition. The total time of the movie (14 minutes) was divided into 2 blocks of 7 minutes with a break between the blocks, to decrease the influence of drowsiness. The whole movie was shown twice: once the subject was asked to observe the movement and once the subject was asked to imagine the movement. The order of observation (obs) and observation-andimagination (obs-and-ima) was randomized between subjects.

During obs-and-ima, subjects were asked to imagine the movement of the hand while watching the movement on the video. This combination of observation and imagination made it possible to directly compare the observation task to the imagination task, since the timing and the cue to imagine or observe was the same. The baseline fragments consisted of a bouncing dot, and followed each action part. The duration of the baseline was randomized between 4 and 12 seconds. The 
movement shown during the activity fragments consisted of a right hand performing a pincer grip, that is, a movement of the top of the thumb towards the top of the index finger and this activity fragment was not preceded by a cue. The movement was shown against a black background and was performed with a frequency of approximately $1 \mathrm{~Hz}$. It was shown in first person perspective. This movement-fragment had a fixed duration of 5 seconds. For one block, subjects were asked to imagine the execution of the movement while the movement was shown in the video. During the other block, subjects were asked to only observe the movement shown. During the baseline condition, the subjects were instructed to observe the dot.

\section{EEG recording}

EEG was recorded against a virtual common reference, using a 64-channel Refa amplifier (TMSi, The Netherlands) using the modified international 10/20 system for electrode placement. The impedance of all EEG-electrodes was below $5 \mathrm{kOhm}$ to reduce polarization effects. Sampling frequency was $512 \mathrm{~Hz}$. EOG of the right eye was measured to monitor eye movements. To monitor potential actual movements, EMG was recorded over the left and right musculus abductor pollicis brevis (APB), and the right musculus flexor carpi radialis (FCR). To assure that the EEG synchronizes with the movie, a trigger signal was generated at the start of the movie.

\section{Data analysis}

Data analysis was performed offline. All routines were implemented in Matlab (The Mathworks, Inc). The EEG was band-pass filtered between 0.01 and $100 \mathrm{~Hz}$. Movement artifacts were removed whenever the EMG in any of the muscles exceeded $50 \mu \mathrm{V}$. To reject the whole movement artifact, 0.05 seconds surrounding the peak of the artifact and 10 samples at both sides were rejected from the analysis. Artifact rejection was controlled by visual inspection.

Event-related synchronization (ERS) and event-related desynchronization (ERD) were defined as an increased or decreased (respectively) instant power during obs or obs-and-ima compared to the latest 3 seconds of the baseline. To avoid possible effects of the phase-locked component on the ERS, the first second after switch of the fragment was disregarded in the analysis (Kalcher, 1995; Solis-Escalante, 2012). To calculate the power changes, first the EEG was filtered into 3 frequency bands (theta: $4-8 \mathrm{~Hz}$, alpha: 8-13 Hz, beta: $13-25 \mathrm{~Hz}$ ) with a 256th order zero phase shift FIR filter. Each fragment was considered an epoch, and the power of each frequency band was subsequently calculated per epoch. 
The average power $(\mathrm{P})$ was calculated as:

$$
P=\frac{1}{N} \sum_{i=1}^{i=n} x_{f(i, j)}^{2}
$$

where $x_{f(i, j)}$ is the $\mathrm{j}$-th sample of the $\mathrm{i}$-th trial of the bandpass filtered data. Subsequently, the ERS was calculated using:

$E R S=\frac{P_{\text {activity }}-P_{\text {rest }}}{P_{\text {activity }}+P_{\text {rest }}}$.

Since baseline and activity movies interchanged, first the ERS (or relative power difference) of each baseline-activity combination was calculated, whereafter the mean of these ERS-values was calculated for each subject, electrode and condition (left and right pincer) separately.

\section{Spatial differences}

The grand average of all subjects was plotted using topoplots. Negative values reflect desynchronization in a particular frequency band during the activity movie compared to baseline.

The differences between obs and obs-and-ima were statistically tested using a repeated measures ANOVA, where the ERS was analyzed using task (obs or obsand-ima) as within-subject variable, and electrode (C3, C4), and band (theta, alpha and beta) as between-subject variables.

In addition, the ERDS from 4-25 Hz was plotted. The difference of the ERDS between obs and obs-and-ima at the peak-values was tested using a repeated measures ANOVA, where task (obs or obs-and-ima) was the within-subject factor, and frequency and electrode the between-subject factors. Post-hoc analyses were done using a paired t-test. Significance level was chosen to be $p=0.05$, which was adjusted by using the Bonferroni-correction for multiple testing. As the Bonferroni-correction may be too conservative, the actual p-values are reported to examine the trends towards significance, as well (Burgess and Gruzelier, 1999).

\section{Temporal differences}

To analyze the temporal changes in EEG power, time-frequency plots were created using a short time Fourier analysis. The width of the window used was $1 \mathrm{~s}$ with an overlap of 15/16 (0.9375). The median signal amplitude measured during the last 3 seconds of each rest fragment was used as baseline, and subtracted from the data measured during obs or obs-and-ima of the hand. To visualize the temporal power changes, the time-frequency plots of $\mathrm{C} 3, \mathrm{C} 4$ and $\mathrm{Pz}$ are shown.

When a frequency band shows major changes, the behavior of the ERS over time was separately plotted for a more detailed examination. Temporal differences were statistically tested using a repeated measures ANOVA, where differences 
between the tasks on time-intervals of 1 second were tested. Post-hoc analyses were done using a paired t-test.

\subsection{Results}

Figure 1 shows the relation between ERS during obs and the ERS during obs-andima on C3 for each subject. The figure shows a scattered distribution of the ERS, indicating large differences between subjects and within subjects.

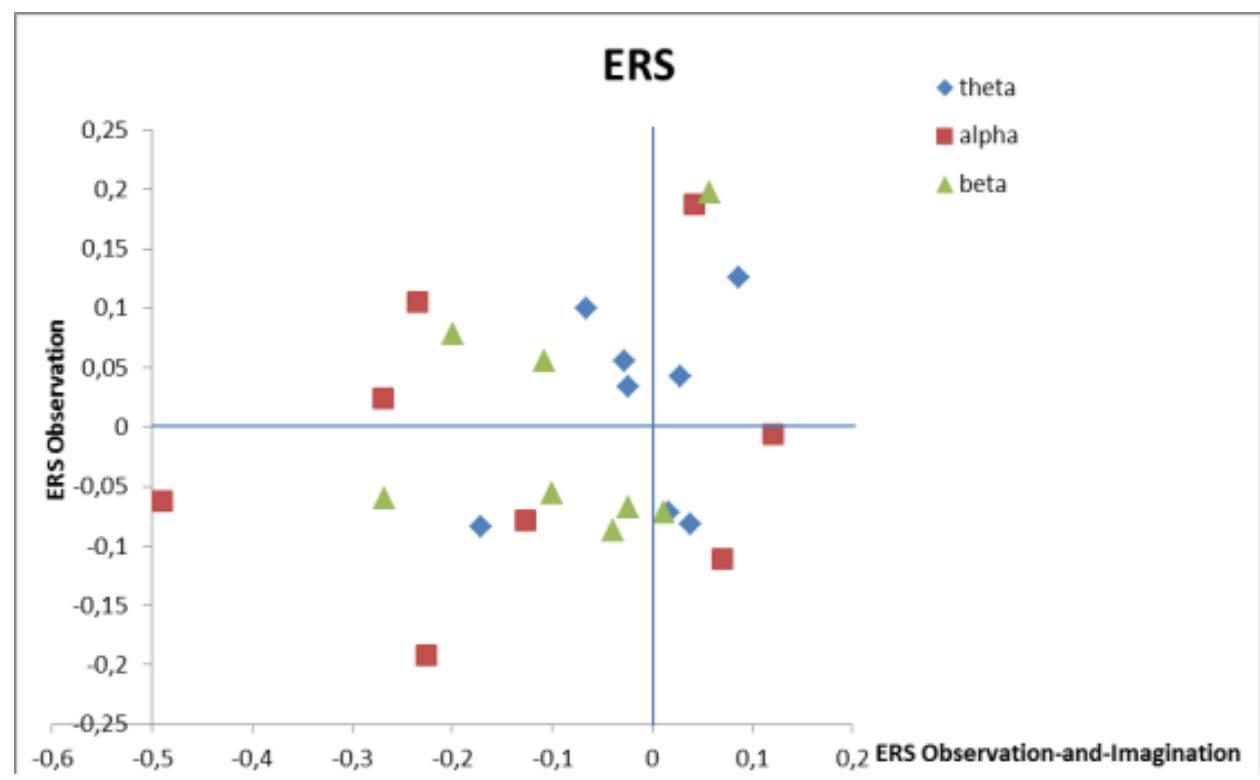

Fig1 Scatterplot showing the ERS on C3 during obs and obs-and-ima of all subjects for the three frequency bands considered theta, alpha and beta. A scattered distribution of the ERS indicates large differences between subjects and within subjects.

\section{Spatial differences}

Despite the large variability between and within subjects, using a repeated measures ANOVA a main-effect for task (obs or obs-and-ima) was found ( $\mathrm{F}=9.108$, $\mathrm{p}=0.004)$. The desynchronization during obs-and-ima $(-0.08556+/-0.138)$ was significantly higher compared to obs $(-0.0184+/-0.0942)$.

There were no significant main-effects for electrode or frequency band and no significant interaction effects were found.

The synchronization during obs and obs-and-ima of the movement is depicted in topoplots (Figure 2). 

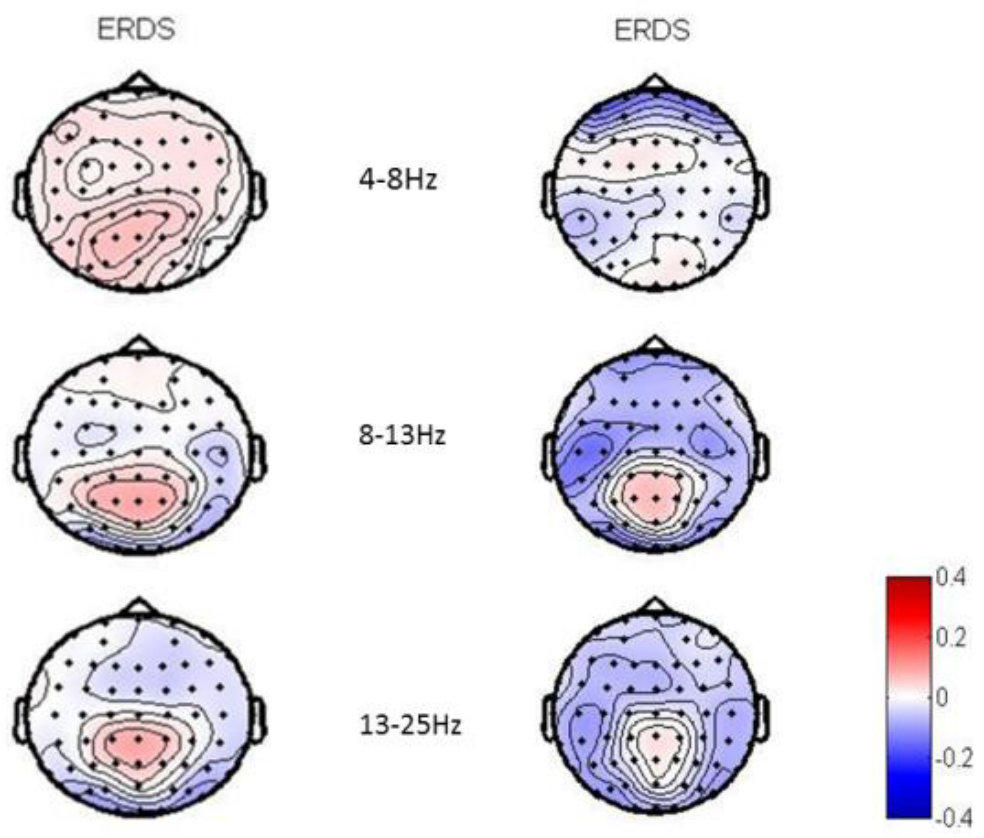

Fig2 Topoplots of the grand average of the (de)synchronization during the tasks. The red color codes a positive (ERS) synchronization, the power of the frequency band increases during the task compared to baseline.

The blue color codes a negative ERS (desynchronization), the power of the frequency band decreases during the task compared to baseline.

During obs-and-ima a larger desynchronization can be found in all frequency bands compared to obs.

\section{Theta $(4-8 \mathrm{~Hz})$}

During obs, the global power increased (ERS), while during obs-and-ima a desynchronization was found in most of the cortical areas (ERD), except occipitally and at the left frontalcentral area.

\section{Alpha $(8-13 \mathrm{~Hz})$}

During both obs-and-ima and obs a desynchronization was found on C3 and C4 although more pronounced during obs-and-ima. At the (centro-)parietal electrodes, $\mathrm{CPz}$ and $\mathrm{Pz}$, a large synchronization was found during both tasks. 


\section{Beta $(13-25 \mathrm{~Hz})$}

During obs-and-ima an ERD was found at C3 and C4. In accordance with the alpha frequency band, the power of the beta frequency band, measured at electrodes $\mathrm{CPz}$ and $\mathrm{Pz}$, increased during both movement obs and obs-and-ima, compared to the baseline condition.

For both obs-and-ima and obs-only, the alpha and beta frequency bands show almost the same pattern of synchronization and desynchronization. During obsand-ima, however, the modulation was significantly larger for C3 and C4 compared to obs.

To examine the ERS during the latest 4 seconds of the activity movie in more detail, Figure 3 shows the ERS evaluated in the different frequency bands over C3 and C4 of this time epoch.
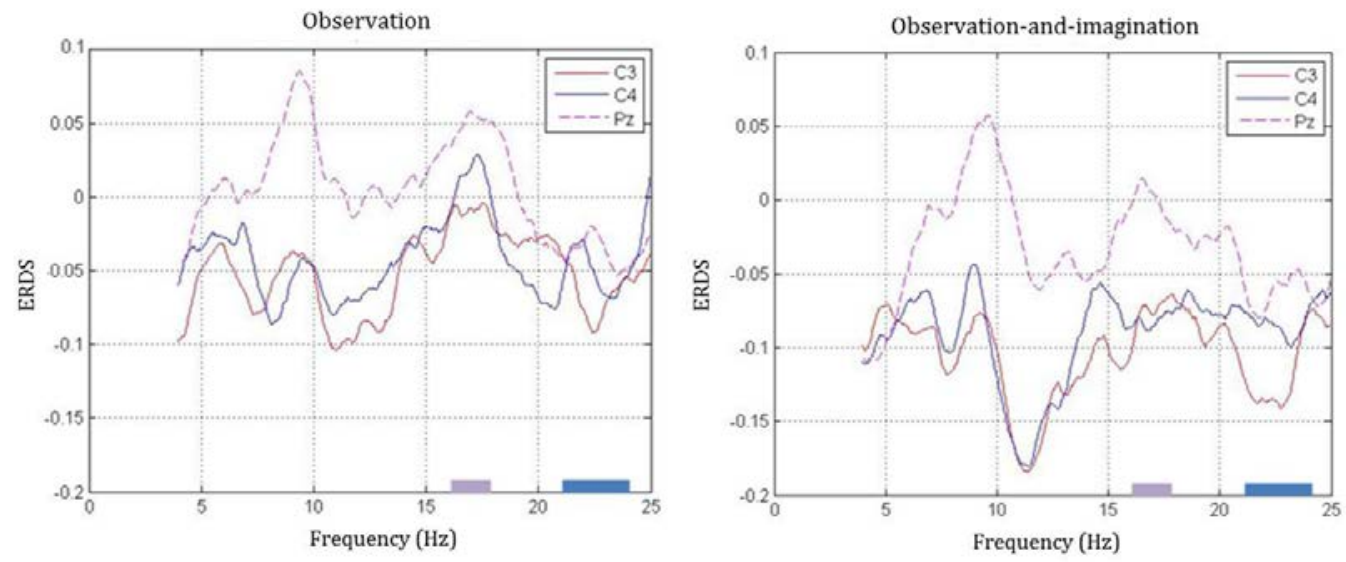

Fig3 The modulation of the ERDS during obs and obs-and-ima in the latest 4 seconds of the activity movie. The thick lines on the $\mathrm{x}$-axis indicate the frequencyintervals where the trends towards significant differences between obs and obsand-ima are found. Purple: Pz; blue: C4.

During obs-and-ima a sharp decrease was found in the frequency band between 10 $\mathrm{Hz}$ and $13 \mathrm{~Hz}$ at both C3 and C4, with a (negative) peak at $11 \mathrm{~Hz}$. At the left (contralateral) hemisphere also a large negative peak was found at $22 \mathrm{~Hz}$.

During obs, at the contralateral side (C3) a small negative peak can also be seen at $11 \mathrm{~Hz}$, although less pronounced compared to obs-and-ima. In the higher frequencies also two small negative peaks can be found at $20 \mathrm{~Hz}$ (C4) and $22 \mathrm{~Hz}$ (C3). In addition, an increased power was found mainly at C4 at $17 \mathrm{~Hz}$.

At Pz 2 positive peaks can be found during both obs and obs-and-ima at $9 \mathrm{~Hz}$ and $17 \mathrm{~Hz}$. 
To examine the differences between obs and obs-and-ima, differences were tested at the low-alpha $\left(\alpha_{1}=8-10 \mathrm{~Hz}\right)$, high-alpha $\left(\alpha_{2}=10.5-11.5 \mathrm{~Hz}\right)$, low-beta $\left(\beta_{1}=16\right.$ $18 \mathrm{~Hz})$ and beta $\left(\beta_{2}=21-23 \mathrm{~Hz}\right)$ frequency bands, using a repeated measures ANOVA. Task (obs and obs-and-ima) was the within subject factor, and frequency bands $\left(\alpha_{1}, \alpha_{2}, \beta_{1}\right.$, and $\left.\beta_{2}\right)$ and electrodes (C3, C4 and Pz) were between-subjects factors. $A$ significant difference between the tasks $(\mathrm{F}=63.77, \mathrm{p}=0.000)$, and an interactioneffect for task and electrode $(\mathrm{F}=3.113, \mathrm{p}=0.047)$ was found.

Post-hoc tests were done using paired t-tests, $\mathrm{p}<0.004$ was considered to be significant. No significant differences between obs and obs-and-ima were found. However, trends towards significance were found at C4 between 21-23 Hz, where obs $(-0.0454 \pm 0.0758)$ is higher compared to obs-and-ima $(-0.08745 \pm 0.0631)$ $(\mathrm{p}=0.025)$. At $\mathrm{Pz}$ a significant difference was found at $16-18 \mathrm{~Hz}$, where obs $(0.0490 \pm 0.137)$ was higher compared to obs-and-ima $(0.00389 \pm 0.0779)$ $(\mathrm{p}=0.012)$.

\section{Temporal differences}

As shown in the topoplots, a strong synchronization was found at $\mathrm{CPz}$ and $\mathrm{Pz}$. Besides the time-frequency analysis (TFA) at C3 and C4, also the temporal behavior at $\mathrm{Pz}$ is described.

During both obs and obs-and-ima the power increased after the task switched from baseline to activity and also vice versa. This synchronization is mainly found in the lower frequency bands.

After this short synchronization, overall, a broader frequency spectrum was found to desynchronize during obs-and-ima compared to obs. In more detail, when subjects were asked to observe-and-imagine the movement, a decreased power was found between $10 \mathrm{~Hz}$ and $14 \mathrm{~Hz}$ (higher alpha frequency band) and between $20 \mathrm{~Hz}$ and $23 \mathrm{~Hz}$ (higher beta frequency band), although this latest power decrease seemed to be stronger at C3 compared to C4. During obs, the desynchronization of the frequency bands between 10 and $14 \mathrm{~Hz}$ can also be seen at C3, although less pronounced compared to obs-and-ima. At C4, during observation mainly the lower alpha frequency band, between $8 \mathrm{~Hz}$ and $10 \mathrm{~Hz}$ desynchronized, compared to a desynchronization of the higher alpha frequency band at C3.

Centro-parietally, at $\mathrm{Pz}$, a synchronization was found in the lower frequency bands after the switch of the fragments, like it was found at C3 and C4. However, in contrast to $\mathrm{C} 3$ and $\mathrm{C} 4$, between $8 \mathrm{~Hz}$ and $10 \mathrm{~Hz}$ the sychronization remained during the whole activity fragment. During obs, this power increase can be found also in the higher frequency bands between 10 and $20 \mathrm{~Hz}$, while during obs-and-ima this increased power was not markedly present. 
The largest modification was found between 11 and $14 \mathrm{~Hz}$, therefore the temporal modifications of this frequency interval were analyzed in more detail (Figure 5)
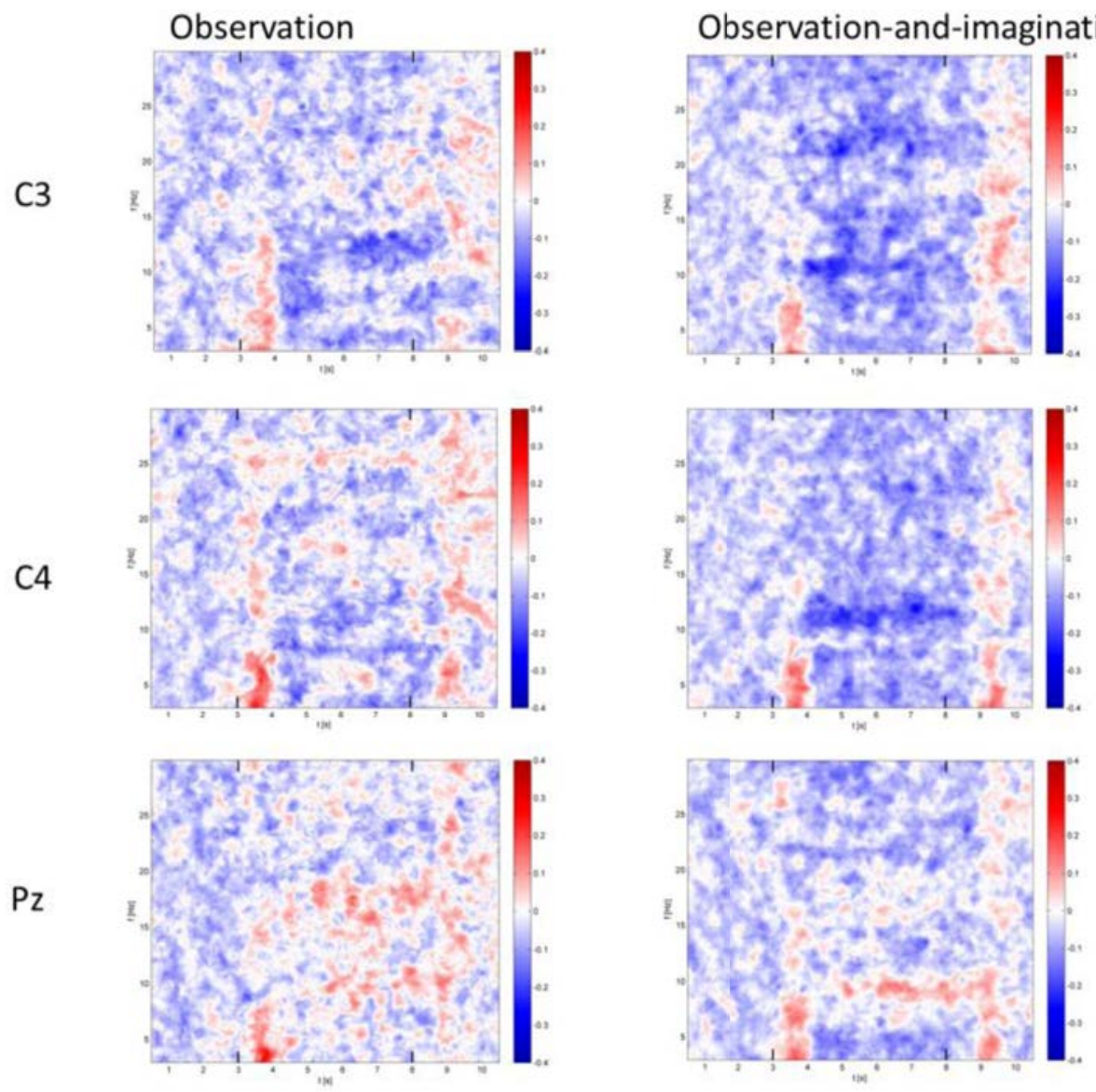

Fig4 Time frequency power plots during obs and obs-and-ima of C3, C4 and Pz. During both obs and obs-and-ima a synchronization was found after the switch of the fragment (at $3 \mathrm{~s}$ switch to activity, at $8 \mathrm{~s}$ switch to baseline). At C3 and C4, the desynchronization during obs-and-ima seems to be larger compared to obs. 
C3

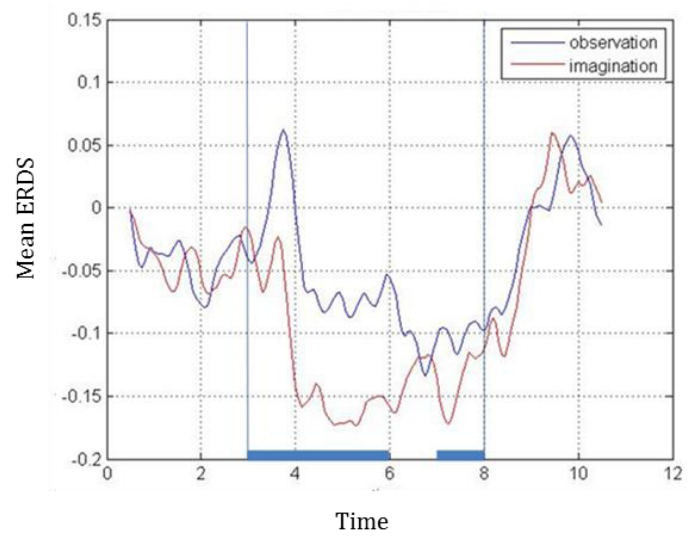

C4

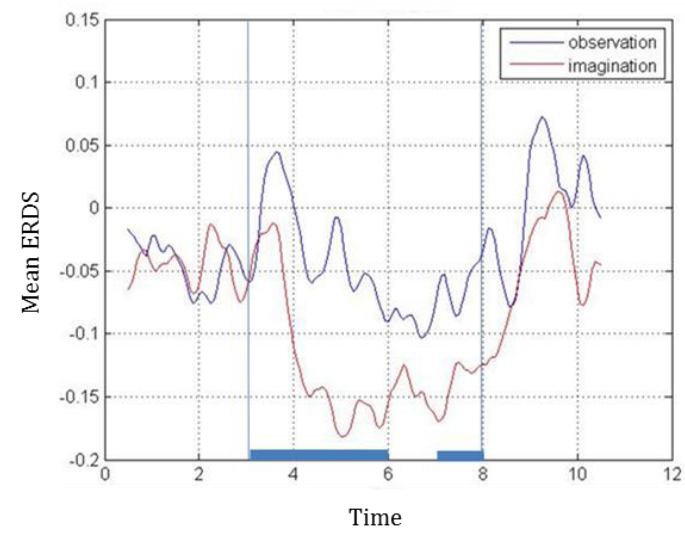

Fig5 Time course of the ERS in the $11-14 \mathrm{~Hz} 14 \mathrm{~Hz}$ frequency range. The first 3 seconds show the baseline. At $\mathrm{t}=3 \mathrm{~s}$ the fragments switch to the activity movie. At 8 seconds the baseline fragments were shown again. The blue colored lines on the $\mathrm{x}$ axis indicate the time-intervals where the trends towards significant differences between obs and obs-and-ima were found.

To statistically test the differences between obs and obs-and-ima, the plot was separated into 9 time-intervals of 1 second $(\mathrm{t} 1=1-2 \mathrm{~s}, \mathrm{t} 2=2-3 \mathrm{~s} \ldots \mathrm{t} 9=9-10 \mathrm{~s})$. A repeated measures ANOVA was subsequently performed for each interval, where time-interval and task (observation and imagination) were within subject variables.

Main-effects were found for task $(\mathrm{F}=5.777, \mathrm{p}=0.033)$ and time-interval $(\mathrm{F}=13.383$, $\mathrm{p}=0.000)$. In accordance with the topoplots, obs-and-ima was significantly lower ($0.113 \pm 0.123)$ than obs $(-0.0463 \pm 0.0067)$. Differences between the time-intervals were found between $\mathrm{t} 3$ and $\mathrm{t} 5(\mathrm{p}=0.035027), \mathrm{t} 3$ and $\mathrm{t} 6(\mathrm{p}=0.045)$ and $\mathrm{t} 3$ and $\mathrm{t} 7$

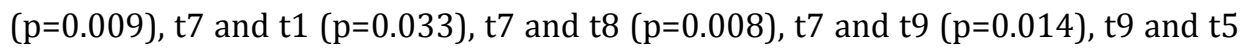
$(\mathrm{p}=0.029), \mathrm{t} 9$ and t6 $(\mathrm{p}=0.029)$. An interaction effect of task and time was also significant $(F=3.255, p=0.004)$. No interaction effects for electrodes were found.

Post-hoc tests were done using a paired t-test, where the significance level was Bonferroni corrected: $\mathrm{p}=0.0055$. No significant differences between observation and imagination were found. Trends towards significance were found: $t 3: p=0.022$ (obs: $0.0062 \pm 0.050$, obs-and-ima: $-0.054 \pm 0.084$ ), $\mathrm{t} 4: \mathrm{p}=0.011$ (obs: $-0.049 \pm 0.068$, obs-and-ima:-0.17 \pm 0.15 ), t5: $\mathrm{p}=0.014$ (obs: $-0.066 \pm 0.087$, obs-and-ima:

$-0.18 \pm 0.15$ ), and t7: $p=0.042$ (obs: $-0.081 \pm 0.070$, obs-and-ima: $-0.17 \pm 0.12$ ) (Figure 5). 


\subsection{Discussion}

During observation-and-imagination, a significant larger desynchronization was found compared to observation-only. This was found in all frequency bands and electrodes and during almost the whole activity fragment. These results suggest a functional difference in brain states between the two tasks.

Although the alpha frequency band is often considered as one, it can be divided into 2 or even 3 bands: the lower, intermediate and higher alpha frequency band. In a study of Klimesch et al (1998), the lower and intermediate alpha bands (8$10 \mathrm{~Hz}$ ) are considered to reflect phasic alertness and expectancy, while the upper alpha frequency band reflects the performance of the tasks. Although the difference was not statistically tested, data suggest that the higher alpha band is desynchronized during imagination where the lower alpha band is desynchronized during observation. When the classification of the alpha frequency band of Klimesch is considered, imagination should relate more closely to performance compared to observation, which only requires alertness. Intuitively this is not surprising, since imagination is more closely related to performance compared to observation. In addition, during imagination the arousal is possibly increased. What is surprising is that the difference is only found in the ipsilateral hemisphere, while at the contralateral side (C3) most desynchronization was found in the higher alpha and higher beta frequency bands during both imagination and observation.

For the higher beta frequency band, a previous study (Bai et al 2008) found that the desynchronization becomes bilaterally symmetrical just before the execution of a movement, suggesting a role in the planning of the movement. The present study found a larger desynchronization of the beta frequency band during obsand-ima, although no cue was used to introduce the activity fragment. In accordance with the study of Bai, this might be explained by the planning of the movement, since imagination requires more planning compared to observation. In the study of Sabate (2012) the preparation for a passive observation of a movement was compared with the preparation of the execution of a movement. They found a higher response during motor planning compared to passive observation, making them to suggest that "the task associated with stimuli is a relevant variable for visually-induced Mu-rhythm suppression". The present study found a higher desynchronization during obs-and-ima compared to obs, and this might underline the suggestion of Sabate et al (2012), since the tasks can be segregated by the amount of desynchronization. Considering these explanations by Bai (2008) and Sabate (2012), it can be expected that the power of the beta frequency band increases after the planning phase, while in the present study the desynchronization remains during the whole activity fragment. The relation between beta frequency desynchronization and high-focus and concentration 
(Pfurtscheller, 1997) might better explain the differences found in the present study between obs and obs-and-ima, since imagination requires more concentration compared to observation. The spatial results, depicted in the topoplots, agree with this suggestion, and also other studies suggest a difference in effort between the two tasks (Grafton et al 1996, Filimon 2007).

Immediately after the switch from baseline to action, and also vice versa, a short synchronization was found in all frequency bands. The short increase in power after a preparation cue of the mu-alpha band was previously published by Kalcher et al (1995), who found a phase-locked increased response of mu-alpha $(8-12 \mathrm{~Hz})$ activity which started $\approx 50 \mathrm{~ms}$ after stimulus onset and persisted for $150-200 \mathrm{~ms}$. Although the presence of alpha activity might indicate a resting (or idling) state of the brain, the lower alpha bands are also suggested to be present during expectancy and alertness. The present study did not use a preparation cue, however the switch of the fragment tells the subject to come into (mental) action, which might cause the increase of the alpha frequency band. Additionally, the short increase of the beta frequency band might be caused by the suppression of the actual movement of the hand (Zhang, 2008). The beta band synchronized after obs and obs-and-ima stopped and the baseline fragment appeared. This might be compared to the "post-movement-beta-synchronization". This post-movementbeta-synchronization is previously found after the execution of a movement and has been interpreted as a correlate of "idling" motor cortex neurons (Pfurtscheller, 1996).

The theta rhythm is associated with drowsiness, and sleepiness. Instead of an increase shortly after the switch of the fragment, an increase of this rhythm could be expected at the end of a fragment. However, a modulation of the parietal (Perfetti, 2011) and frontal (Tombini, 2009) theta activity during a movement task has been linked to cognitive or attentional resources and seem to link spatial (attentional) selection of a target, in order to capture it with a movement (Rawle et al, 2012). This might explain the synchronization of the power of the theta frequency band at the start of the fragments. However, since this synchronization occurs during both obs and obs-and-ima, this result is not caused by the addition of imagination to observation-only.

Synchronization at centro-parietal areas.

The centroparietal synchronization neither discriminates between obs and obsand-ima and the synchronization appears to be caused by the observation. Molenberghs et al (2010), found a significant activity of the left superior parietal lobule, and also in the left supramarginal gyrus, left dorsal premotor area and bilateral superior temporal sulcus, using fMRI. These signs indicate a function of the parietal lobule in imitation and observation. Although this seems to be in contrast with our results, the relationship between the event related 
synchronization measured by EEG and the blood flow, or BOLD signal is still under debate (Rosa, 2010).

Studies examining the cortical activity of pistol shooters and golf players also found a parietal alpha ERS (Del Percio, 2009; Del Percio, 2011; Babiloni, 2011). It has been suggested that parietal alpha ERS reflects a focus on visuo-spatial attention. A diffuse parietal alpha ERS is found in combination with focused alpha ERD in the small region of the cortex related to the spatial area where the attention is focused. In the present study, the subjects were asked to observe or imagine the presented movements on the screen. It is likely that subjects pay extra attention during the movement fragment compared to the bouncing ball, resulting in the diffuse parietal alpha ERS.

In sum, ERD of the sensorimotor cortex is significantly higher during observationand-imagination compared to observation only. The increased desynchronization during observation-and-imagination is found in all frequency bands and remains during the whole activity period. Assuming that a larger ERD is correlated to a larger participation of neurons that are (presumably) also activated by actual movement, these findings suggest an additive effect of imagination instead of observation only in the rehabilitation after stroke. 


\subsection{References}

Babiloni C, Babiloni F, Carducci F, Cincotti F, Cocozza G, Del PC, et al (2002) Human cortical electroencephalography (EEG) rhythms during the observation of simple aimless movements: a high-resolution EEG study. Neuroimage 17:559-572

Babiloni F, Cincotti F, Bianchi L, Pirri G, Del RM, Mourino J, et al (2001) Recognition of imagined hand movements with low resolution surface Laplacian and linear classifiers. Med Eng Phys 23:323-328

Babiloni C, Infarinato F, Marzano N, Iacoboni M, Dassù F, Soricelli A, et al (2011) Intrahemispheric functional coupling of alpha rhythms is related to golfer's performance: A coherence EEG study. In Jour of Psychophys 82:260-268

Bai O, Lin P, Vorbach S, Floeter MK, Hattori N, Hallett M (2008) A high performance sensorimotor beta rhythm-based brain-computer interface associated with human natural motor behavior. J Neural Eng 5:24-35

Calmels C, Holmes P, Jarry G, Leveque JM, Hars M, Stam CJ (2006) Cortical activity prior to, and during, observation and execution of sequential finger movements. Brain Topogr 19:7788

Cochin S, Barthelemy C, Roux S, Martineau J (1999) Observation and execution of movement: similarities demonstrated by quantified electroencephalography. Eur J Neurosci 11:1839-182

Del Percio C, Babiloni C, Bertollo M, Marzano N, Iacoboni M, Infarinato F, et al (2009) Visouattentional and sensorimotor alpha rhythms are related to visuo-motor performance in athletes. Hum Brain Mapp 30:3527-3540

Del Percio C, Iacoboni M, Lizio R, Marzano N, Infarinato F, Vecchio F, et al (2011) Functional coupling of parietal alpha rhythms is enhanced in athletes before visuomotor performance: a coherence electroencephalographic study. Neurosci 175: 198-211

Dijkerman HC, Ietswaart M, Johnston M, MacWalter RS (2004) Does motor imagery training improve hand function in chronic stroke patients? A pilot study. Clin Rehabil 18:538-549

Ertelt D, Small S, Solodkin A, Dettmers C, McNamara A, Binkofski F, et al (2007) Action observation has a positive impact on rehabilitation of motor deficits after stroke. NeuroImage 36 suppl2: T164-T173

Filimon F, Nelson JD, Hagler DJ, Sereno MI (2007) Human cortical representations for reaching: mirror neurons for execution, observation, and imagery Neuroimage 37:13151328

Grafton ST, Arbib MA, Fadiga L, Rizzolatti G (1996) Localization of grasp representations in humans by positron emission tomography. Exp Brain Res 112:103-111 
Jeannerod M (2001) Neural simulation of action: a unifying mechanism for motor cognition. Neuroimage 14: S1-3-S109

Kalcher J, Pfurtscheller G (1995) Discrimination between phase-locked and non-phaselocked event-related EEG activity. Electroencephalogr Clin Neurophysiol 94: 381-384

Klimesch W, Doppelmayr M, Russegger H, Pachinger T, Schwaiger J (1998) Induced alpha band power changes in the human EEG and attention. Neursci Let 244:73-76

Léonard G, Tremblay F (2007) Corticomotor facilitation associated with observation, imagery and imitation of hand actions: a comparative study in young and old adults. Exp Brain Res 177(2):167-175

Liepert J, Hassa T, Tüscher T, Schmidt R (2011) Motor excitability during movement imagination and movement observation in psychogenic lower limb paresis. J Psychosom Res 70(1):59-65

Molenberghs P, Brander C, Mattingley JB, Cunnington R (2010) The role of the superior temporal sulcus and the mirror neuron system in imitation. Human Brain Mapping 19:1-11

Mulder T, de Vries S, Zijlstra S (2005) Observation, imagination and planation of motor imagery. Exp Brain Res 163: 344-351

Page SJ, Levine P, Leonard (2007) Mental practice in chronic stroke: results of a randomized, placebo-controlled trial. Stroke 38:1293-1297

Pfurtscheller G, stancák A Jr, Neuper C (1996) Post-movement beta synchronization. A correlate of an idling motor area? Electroencephalogr clin Neurophysiol 98:281-293

Pfurtscheller G, Neuper C, Andrew C, Edlinger G (1997) Foot and hand area mu rhythms. Int J Psychophysiol 26:121-135

Pfurtscheller G, Lopes da Silva FH (1999) Event-related EEG/MEG synchronisation and desynchronisation: basic principles. Clin Neurophysiol 110:1842-1857

Pleger B, Schwenkreis P, Grünberg C, Malin JP, Tegenthoff M (2004) Fluoxetine facilitates use-dependent excitability of human primary motor cortex. Clin Neurophysiol 115:21572163

Rizzolatti G, Craighero L (2004) The mirror-neuron system. Annu Rev Neurosci 27:169-192

Roosink M, Zijdewind I (2010) Corticospinal excitability during observation and imagery of simple and complex had tasks: implications for motor rehabilitation. Behav Brain Res 12:213(1):35-41 
Rosa MJ, Kilner J, Blankenburg F, Josephs O, Penny W (2010) Estimating the transfer function from neuronal activity to BOLD using simultaneous EEG-fMRI. Neuroimage 49:1496-1509

Sabate M, Llanos C, Enriquez E, Rodriguez M (2012) Mu rhythm, visual processing and motor control. Clin Neurophys. 123:550-557

Schulte-Rüther M, Markowitsch HJ, Fink GR, Piefke M (2007) Mirror neuron and theory of mind mechanisms involved in face-to-face interactions: a functional magnetic resonance imaging approach to empathy. J Cogn Neurosci 19:1354-1372

Schuster C, Butler J, Andrews B, Kischka U, Ettlin T (2012) Comparison of embedded and added motor imagery training in patients after stroke: results of a randomized controlled pilot trial. Trials $23 ; 13: 11$

Solis-Escalante T, Muller-Putz GR, Pfurtscheller G, Neuper C (2012) Cue-induced beta rebound during withholding of overt and covert foot movement. Clin Neurophysiol 123:1182-1190 2012

Tombini M, Zappasodi F, Zollo L, Pellegrino G, Cavallo G, Tecchio F, Guglielmelli E, Rossini PM (2009) Brain activity preceding a 2D manual catching task. NeuroImage 47:1735-1746

De Vries S, Mulder T (2007) Motor imagery and stroke rehabilitation: a critical discussion. J Rehabil Med 39:5-13

Zhang Y, Chen Y, Bressler SL, Ding M (2008) Response preparation and inhibition: The role of the cortical sensorimotor beta rhythm. Neurosci 156:238-246 



\section{CHAPTER 6}

\section{Influence of a single dose of fluoxetine on brain activity patterns during imagination and execution of a movement in chronic stroke patients}

Berends HI, Movig KLL, IJzerman MJ, Van Putten MJAM

Submitted to Clinical Neurophysiology 


\subsection{Abstract}

Background

Relearning of lost functions after stroke depends on neuroplasticity. Two interventions that may promote neuroplasticity are the use of psychoactive drugs and motor imagery training.

\section{$\underline{\text { Objective }}$}

To examine the effects of a single dose of fluoxetine on brain activity during movement execution and movement imagination in chronic stroke patients.

\section{Methods}

This study was a randomized, double-blind, placebo-controlled, crossover trial. Ten patients were assigned to fluoxetine $20 \mathrm{mg}$ and placebo, or vice versa. To induce motor imagination, a video was shown. Patients watched 64 fragments with a hand performing a pincer grip alternated with a baseline condition. In the execution condition patients had to perform the pincer grip movement. We recorded a 64-Channel EEG, and quantified event related (de-)synchronization (ERS) at C3 and C4 in the theta, alpha and beta frequency bands. Statistically significant differences between fluoxetine and placebo during imagination and execution were evaluated using paired t-tests.

\section{$\underline{\text { Results }}$}

During both movement and imagination, event related desynchronization (ERD) was observed in all frequency bands. When executing a movement by the paretic hand, the ERD of the theta, alpha and beta frequency bands was higher in the affected (contralateral) hemisphere compared to imagination $(p=0.018)$. After fluoxetine a significantly larger ERD in the alpha frequency band was found in the affected (ipsilateral) hemisphere during execution of the healthy hand $(p=0.049)$, but not during imagination.

\section{$\underline{\text { Conclusion }}$}

ERD of the frequency bands over the motor cortex indicates the ability of stroke patients to imagine a movement of the paretic arm while the execution of the movement is barely possible. Fluoxetine did not affect this desynchronization during the movement or imagination of the movement of the affected hand. However, the increased ERD over the paretic cortex during movement of the healthy hand after fluoxetine indicates the possibility of a single dose to affect cortical activity. 


\subsection{Introduction}

Stroke has an invalidating effect on the motor function of many patients, even after long and intensive rehabilitation therapies. During rehabilitation, improvement of the daily live functioning of the stroke patients can be achieved by training of the lost functions, and/or by the training of compensatory techniques (Kwakkel, 2004; Cirstea, 2000). The relearning of lost functions depends on the adaptation of the brain, resulting from neuroplasticity. Neuroplasticity includes all processes that modulate brain function by shaping existing synaptic connectivities or promote new connections. Improvement of regular physical training therapies might be established by using interventions facilitating neuroplasticity. Two possible interventions to promote neuroplasticity are the use of psychoactive drugs and motor imagery training (Chollet, 2011).

During physical training, neuroplasticity of the brain occurs by axonal or dendritic sprouting (re)establishing corticocortical-or-corticospinal connections. In stroke, neuroplasticity is also presumed to be induced by physical training, causing activation of intact neurons adjacent to the infarcted site (Krakauer, 2006). However, physical training is often time-consuming and exhausting for the patient, because many repetitions have to be done before any improvement is expected. One possibility to facilitate this therapy is by using imagination or observation of a movement. During observation in order to imitate a movement, some brain areas are activated which are also activated during the execution of a movement (Calmels, 2006). Theoretically, motor performance can be improved through systematic exercise based on careful observation and imitation of everyday actions (Buccino, 2006; Berends, 2009). Studies examining the effects of action observation (Ertelt, 2007) or mental practice (Page, 2007) on the rehabilitation of motor function after stroke support this theory. These studies showed an improved motor function after the observation of a movement, or after mental practicing of the movement, while motor areas were found to be reactivated. Motor imagery training demands less physical effort and may improve outcome in stroke patients.

Another intervention that may promote neuroplasticity after stroke is the use of psychoactive drugs. Single doses of SSRI (20 $\mathrm{mg}$ fluoxetine, and $20 \mathrm{mg}$ paroxetine) were found to cause a more focused activation in the contralateral sensorimotor area, a greater involvement of the posterior supplementary motor area, and a widespread decrease of bilateral cerebellar activation in an active motor task (Loubinoux, 1999; Loubinoux, 2002). In addition, after intake of a single dose of an SSRI (fluoxetine $(20 \mathrm{mg}$ ) (Pleger, 2004), and sertraline (100mg) (Ilic, 2002)) an increased excitability of the motor cortex (Pleger, 2004), corticospinal neurons and spinal motor neurons was found in healthy subjects, without a change in motor threshold (MT). However, also an inhibitory effect of sertraline was found in the activity of excitatory interneurons that project onto the corticospinal neuron. After 
stroke a single dose of fluoxetine causes a hyperactivation in the ipsilesional primary motor cortex, during an active motor task (finger tapping test). In addition, it is found to increase muscle activation (Pariente, 2001) and to affect motor skills on the lesioned side. Another SSRI (citalopram) is found to improve motor performance after a single dose (Zittel, 2008). A Cochrane review by Mead et al "provided evidence of benefit of SSRI for reducing disability and neurological impairment scores in people with stroke." They concluded that SSRIs appeared to improve dependence, disability, neurological impairment, anxiety and depression after stroke (Mead, 2013).

After long-term use of $20 \mathrm{mg}$ of fluoxetine a day for 3 months, Chollet et al found an improved motor function, measured using the Fugl Meyer motor assessment (FM) (Chollet, 2011).

After administration of fluoxetine, the reuptake of serotonin in the synaptic cleft between neurons in the brain is inhibited, causing an increased amount of serotonin concentrations in the brain. This increased amount is found to have a use-dependent facilitating effect on motor cortex excitability (Pleger, 2004), and to increase the excitability of cortico-spinal neurons and spinal motoneurons (Ilic, 2002). Previous studies found a possible relation between an increased excitability and an increased event related desynchronization (Pfurtscheller, 1999; Mäkelä, 2015). In addition, a relation was found between a reduction of beta synchronization and clinical improvement after physiotherapy of patients with chronic stroke (Wilson, 2011). In sum, it can be expected that an increased excitability (related to event-related desynchronization) correlates with motor outcome. Thereby, serotonin is thought to facilitate motor output (Jacobs 1999; Pariente 2001). By examining the ERS and ERD of the theta, alpha and beta frequency bands during execution and imagination, we try to better understand the effects of the previously found increased excitability.

The objective of our study was to examine the effects of a single dose of fluoxetine on brain activity during movement execution and movement imagination in chronic stroke patients.

\subsection{Methods}

Subjects and setting

The study was performed in Roessingh Research and Development, and a large revalidation teaching hospital in Enschede, the Netherlands. From 2009 until 2010, we recruited stroke patients from the outpatient clinic of this hospital. Included patients had to be at least 18 years old, and suffering from a single, unilateral stroke confirmed by MRI or CT. Time post stroke had to be over 6 months (chronic stroke). Patients had to have an affected hand and/or arm function, with some voluntary muscle contraction. Exclusion criteria were other (pre-existing) 
neurological diseases (e.g. epilepsy, tumour, paralysis), known allergy to SSRI's, use of anti-depressants, autism spectrum disorders, pervasive development disorder, schizophrenia (or history of schizophrenia), unstable medical health situation, uncompensated hemineglect or cognitive disabilities, resulting in misunderstanding or incapability of executing instructions given, uncorrected visual problems, pregnancy, severe uncontrolled medical conditions, known alcoholism or drug abuse, known elevated brain pressure.

The trial was approved by the institutional ethics committee. The EudraCT number was 2008-003349-97. All patients gave written informed consent.

\section{$\underline{\text { Subjects }}$}

Eleven stroke patients (mean age: $58.5 \pm 11.16$, 3 female) were included. One patient was excluded from further analysis, since measurements were discontinued after notification of a previous seizure. 7 Patients suffered from a lefthemispheric stroke. All patients were right handed before stroke. Although all patients suffered from motor function impairment, some muscle activity was still present. Mean Fugl Meyer Motor Assessment score of the upper extremity part was $42 \pm 18$, with a minimum score of 14 and a maximum score of 60 . The maximum score of the upper extremity part of the FM is 66.

\section{Study design}

The study was designed as a randomized, double-blind, placebo-controlled, crossover trial.

Patients were randomly assigned to a crossover treatment: they were assigned to fluoxetine $20 \mathrm{mg}$ and placebo, or vice versa. Fluoxetine was dispensed as a capsule. The corresponding placebo capsule was identical except for the fluoxetine. Before the trial, randomization was performed by an independent statistician. The study medication was produced and labeled before the start of the trial by an independent hospital pharmacist not otherwise involved in the trial. Patients, doctors, and researchers were unaware of treatment assignment until all patients had completed the study and all data were analyzed.

\section{Measurements}

Since the half-life of fluoxetine is 2 days, all measurements were done on 2 days separated by 2 weeks in order to minimize the effects of a previous gift. Measurements were started 5 hours after intake of fluoxetine or placebo.

To induce motor imagination, a video was shown consisting of 64 fragments showing an aimless hand movement and a baseline condition intermittently. To decrease the influence of drowsiness, the total time of the movie (14 minutes) was divided into 2 blocks of 7 minutes with a break between the blocks. This combination of observation and imagination was used because of earlier positive 
results in healthy subjects (Berends, 2013). Additionally, the stroke patients were thought to better be able to concentrate on the movement when observing it.

The activity fragment showed the movement of a hand performing a pincer grip (a movement of the top of the thumb towards the top of the index finger). First the movement of the non-affected hand was shown followed by the movement of the affected hand. Frequency of the movements, shown against a black background, was approximately $1 \mathrm{~Hz}$. The fragment was shown in first person perspective and was not preceded by a cue. The activity-fragment had a fixed duration of 5 seconds. Subjects were asked to imagine the execution of the movement while the movement was shown. The baseline condition, video fragments following each activity fragment, consisted of a bouncing dot. The duration of the baseline was randomized between 4 and 12 seconds. During the baseline condition, the subjects were instructed to observe the dot (Figure 1).

In the execution condition, the patient had to make the pincer grip movement by his- or herself. The execution of the movement was done $10 \times 5$ seconds alternated between both hands. As baseline condition the EEG was measured for 1 minute while the patient was seated in front of a black screen.

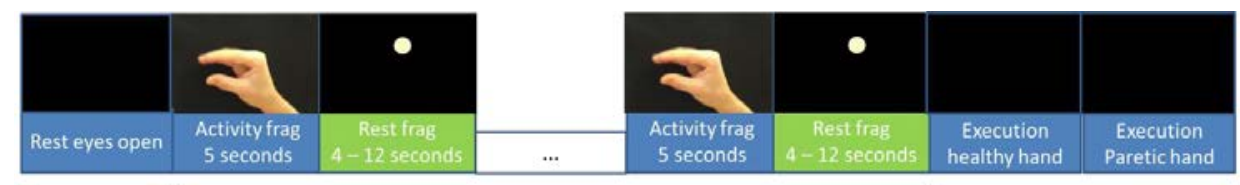

1 minute

Figure 1. Schematic representation of the measurement procedure.

\section{EEG recording}

EEG was recorded against a virtual common reference, using a 64-channel Refa amplifier (TMSi, The Netherlands) using the modified international 10/20 system for electrode placement. The impedance of all EEG-electrodes was below $5 \mathrm{kOhm}$ to reduce polarization effects. Sampling frequency was $512 \mathrm{~Hz}$. EOG of the right eye was measured to monitor eye movements. To monitor actual movements, EMG was recorded over the left and right musculus abductor pollicis brevis (APB), and the right musculus flexor carpi radialis (FCR). To assure that the EEG synchronizes with the video, a trigger signal was generated at the start.

\section{Data analysis}

The EEG was band-pass filtered between 0.01 and $100 \mathrm{~Hz}$. Movement artifacts were removed whenever the EMG in any of the muscles exceeded $50 \mu \mathrm{V}$ during observation of the movement. To reject the whole movement artifact, 0.05 seconds 
surrounding the peak of the artifact and 10 samples at both sides were rejected from the analysis. Artifact rejection was controlled by visual inspection.

Changes in cortical activity were defined by changes in the power spectrum of the EEG. Event-related synchronization (ERS) and event-related desynchronization (ERD) were defined as an increased or decreased (respectively) instant power during execution or imagination compared to rest. For imagination, the last 3 seconds of the baseline movie was defined as rest. To avoid possible effects of the phase-locked component on the ERS, the first second after switching of fragments was disregarded in the analysis (Kalcher, 1995; Solis-Escalante, 2012). For execution the power of the frequency bands during "rest-with-eyes-opened" was used, since the patients were found not to be able to relax the muscles in between two execution comments. To calculate the power changes, the EEG was filtered into 3 frequency bands (theta: 4-8 Hz, alpha: 8-13 Hz, beta: $13-25 \mathrm{~Hz}$ ) with a 256th order zero phase forward and reverse FIR filter. Each fragment was considered an epoch, and the power of each frequency band was subsequently calculated per epoch. The average power, $\mathrm{P}$, was calculated as

$P=\frac{1}{N} \sum_{i=1}^{i=n} x_{f(i, j)}^{2}$

with $x_{f(i, j)}$ the $\mathrm{j}$-th sample of the trial i of the bandpass filtered data.

Subsequently, the ERS was calculated using

$E R S=\frac{P_{\text {activity }}-P_{\text {rest }}}{P_{\text {activity }}+P_{\text {rest }}}$.

To analyze the ERS during imagination, first the ERS (or relative power difference) of each baseline-activity combination was calculated, after which the mean of these ERS-values was calculated for each subject, electrode and condition (left and right pincer) separately. A negative ERS implies a decrease of the power of frequency band during the activity movie compared to baseline, and can be defined as event related desynchronization (ERDS). We will further refer to ERDS, as we expect a decrease in power during the 'activity' condition.

To analyze the ERDS during execution, the segments of EEG were selected during which EMG was simultaneously measured in one of the measured muscles. Since the patients were hardly able to relax the muscles after the execution of the movement, the power of the frequency bands during a 1 minute rest with eyes opened was used to calculate ERDS. These data were controlled for EMG activity by visual inspection. 
Additionally to the ERDS, the lateralization of the power, Lpower during the execution and the imagination is defined as

$$
L_{\text {power }}=\frac{P_{\text {contralateal }}-P_{\text {ipsilaterd }}}{P_{\text {contralateal }}+P_{\text {ipsilaterd }}} .
$$

\section{$\underline{\text { Statistical analysis }}$}

Data analysis was performed offline. All routines were implemented in Matlab (The Mathworks, Inc.).

A previous study of our research group presenting significant effects of a single dose of fluoxetine $20 \mathrm{mg}$ on the muscle activity patterns in chronic stroke patients included 10 patients (Berends, 2009). Therefore, we decided to include 10 chronic stroke patients for the present study, using the same in- and exclusion criteria as the previous.

To verify the ability of stroke patients to imagine the movement of the affected hand, a t-test was conducted examining whether the ERDS during imagination of the affected hand significantly differed from 0 after the administration of a placebo. Differences between fluoxetine and placebo

To analyze differences of ERDS after administration of fluoxetine or placebo, paired t-tests were conducted. Separate tests were conducted for arm (healthy/paretic), side (contra-/ipsilateral), hemisphere (affected/non-affected) and frequency band (theta/alpha/beta).The hypothesis to be tested is an increased ERDS in both hemispheres in all frequency bands after fluoxetine compared to placebo. $\mathrm{p}<0.05$ was considered significant.

Differences between lateralization of the frequency bands during execution and imagination were considered significant if $\mathrm{p}<0.05$ using paired t-tests.

\section{Differences between execution and imagination}

To analyze the differences between imagination and execution, the ERDS and lateralization were tested using paired t-tests.

\subsection{Results}

The ERDS at the contralateral (affected) side after the administration of a placebo did not show significant difference compared to 0 . However, the power of the bands measured at the ipsilateral, healthy side significantly desynchronized during the imagination of the movement compared to rest (table 1). 
Table 1: ERDS during the imagination and execution of a hand movement. ERDS of the theta, alpha and beta frequency bands is averaged. The contralateral hemisphere is the affected hemisphere.

\begin{tabular}{|l|l|l|l|l|}
\hline Task & \multicolumn{2}{|l|}{ Intervention } & $\begin{array}{l}\text { ERDS } \\
\text { Contralateral } \\
\text { hemisphere } \\
\text { Mean } \pm \mathrm{sd})\end{array}$ & $\begin{array}{l}\text { ERDS Ipsilateral } \\
\text { Hemisphere } \\
\text { (Mean } \pm \mathrm{sd})\end{array}$ \\
\hline \multirow{2}{*}{ Imagination } & \multirow{2}{*}{ Placebo } & Healthy & $-0.35 \pm 0.31^{*}$ & $-0.29 \pm 0.27^{*}$ \\
\cline { 3 - 5 } & Paretic & $-0.10 \pm 0.32$ & $-0.28 \pm 0.29^{*}$ \\
\cline { 3 - 5 } & \multirow{2}{*}{ Fluoxetine } & Healthy & $-0.32 \pm 0.26^{*}$ & $-0.28 \pm 0.29^{*}$ \\
\cline { 3 - 5 } & Paretic & $-0.17 \pm 0.36^{*}$ & $-0.29 \pm 0.23^{*}$ \\
\hline \multirow{2}{*}{ Execution } & \multirow{2}{*}{ Placebo } & Healthy & $-0.32 \pm 0.29^{*}$ & $-0.33 \pm 0.18^{*}$ \\
\cline { 3 - 5 } & & Paretic & $-0.17 \pm 0.28^{*}$ & $-0.038 \pm 0.26$ \\
\cline { 3 - 5 } & \multirow{2}{*}{ Fluoxetine } & Healthy & $-0.17 \pm 0.30^{*}$ & $-0.33 \pm 0.26^{*}$ \\
\cline { 3 - 5 } & & Paretic & $-0.26 \pm 0.22^{*}$ & $-0.14 \pm 0.38$ \\
\hline
\end{tabular}

*: significant difference between activity and rest

Considering the data in table 1 , the frequency bands in the contralateral and ipsilateral hemisphere desynchronized during the execution and imagination of hand movement compared to rest in most of the tested conditions. This suggests that the patients were able to carry out the given task.

\section{Interventional effects}

No differences were found between baseline activity (rest) after placebo and after fluoxetine.

After administration of fluoxetine a significant higher desynchronization of the alpha frequency band was found in the affected ipsilateral hemisphere during execution of the healthy hand $(\mathrm{p}=0.049)$ (fluoxetine: $-0.069 \pm 0.068$, placebo $0.011 \pm 0.13$ ).

No other statistically significant differences between fluoxetine and placebo were found (Figure 2). 

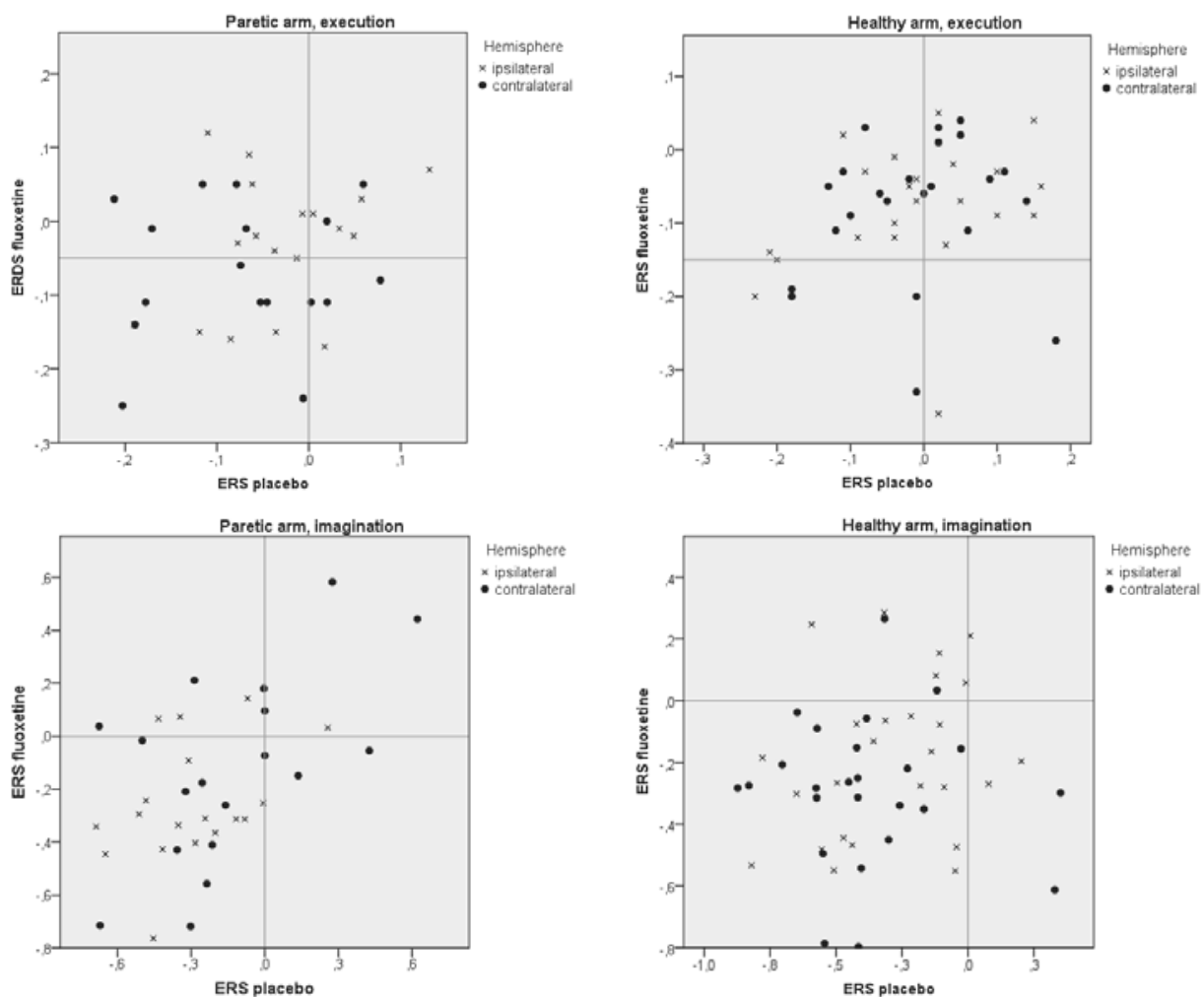

Figure 2: Scatterplots of the relation between ERS after fluoxetine and after placebo. Shown are the ERS values of all frequency bands over the contralateral and the ipsilateral hemisphere ( $\mathrm{C} 3$ and $\mathrm{C} 4$ ) during execution and imagination of the paretic and the healthy hand.

No significant differences were found between the lateralization after fluoxetine and after placebo (Figure 3). 

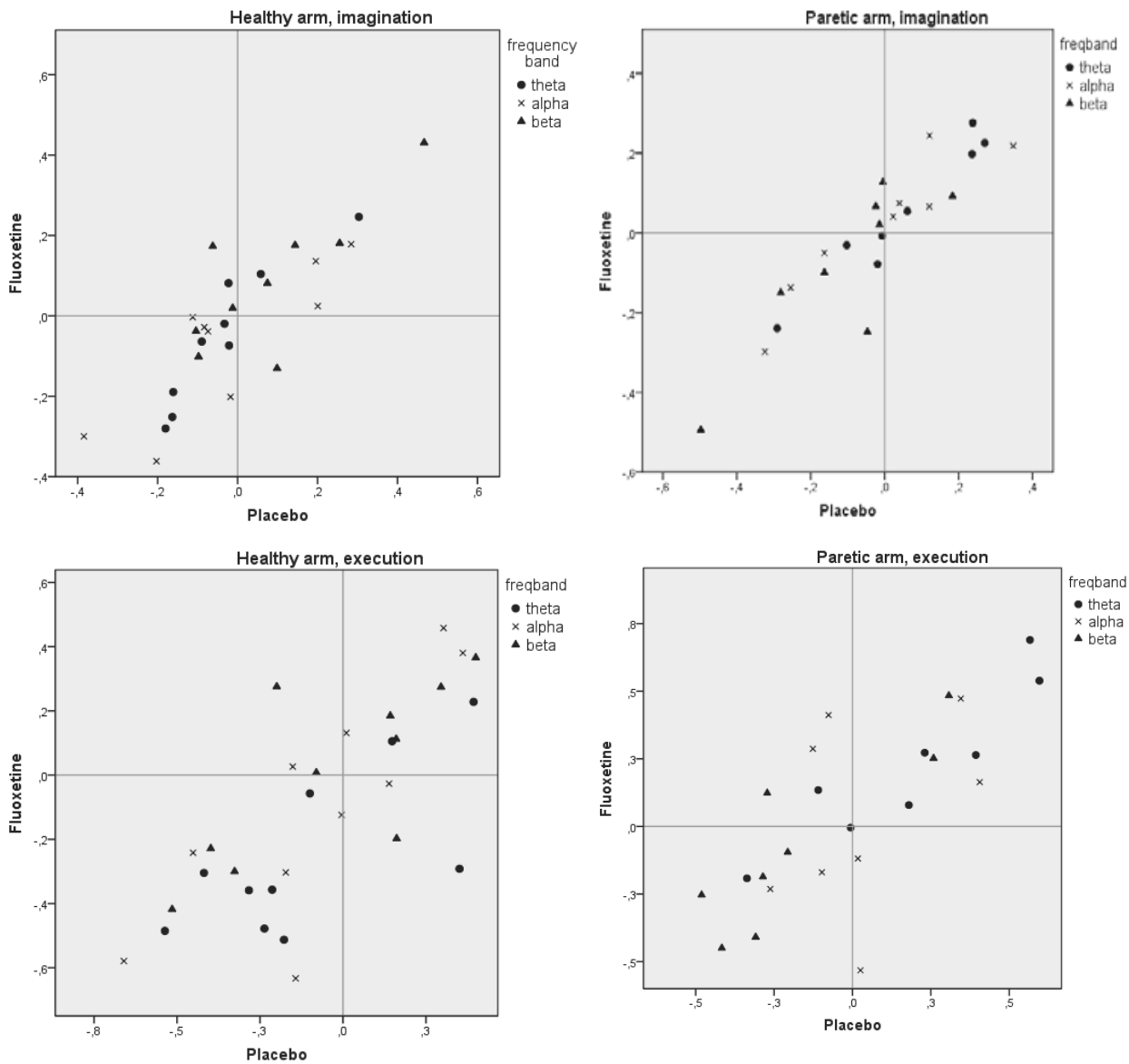

Figure 3: Scatterplots of the lateralization after fluoxetine and placebo. Shown are $\mathrm{L}_{\text {power }}$ after fluoxetine and placebo during imagination and execution of both the healthy and the paretic arm, of each frequency band separately. Lateralization of the theta, alpha and beta frequency bands do not differ after intake of fluoxetine or placebo. 
Table 2: Descriptive statistics of the lateralization

\begin{tabular}{|l|l|l|l|l|}
\hline Arm & $\begin{array}{l}\text { Frequency } \\
\text { band }\end{array}$ & Intervention & $\begin{array}{l}\text { Execution } \\
\text { Mean } \pm \text { sd }\end{array}$ & $\begin{array}{l}\text { Imagination } \\
\text { Mean } \pm \text { sd }\end{array}$ \\
\hline Healthy & Theta & Placebo & $-0.11 \pm 0.31$ & $-0.035 \pm 1.5$ \\
\hline & & Fluoxetine & $-0.25 \pm 0.26$ & $-0.061 \pm 0.17$ \\
\hline Healthy & Alpha & Placebo & $-0.077 \pm 0.32$ & $-0.022 \pm 0.21$ \\
\hline & & Fluoxetine & $-0.091 \pm 0.36$ & $-0.086 \pm 0.19$ \\
\hline Healthy & Beta & Placebo & $-0.036 \pm 0.31$ & $0.085 \pm 0.19$ \\
\hline & & Fluoxetine & $0.0077 \pm 0.28$ & $0.068 \pm 0.18$ \\
\hline Paretic & Theta & Placebo & $0.24 \pm 0.34$ & $0.11 \pm 0.23$ \\
\hline & & Fluoxetine & $0.24 \pm 0.28$ & $0.13 \pm 0.35$ \\
\hline Paretic & Alpha & Placebo & $0.046 \pm 0.22$ & $0.37 \pm 0.83$ \\
\hline & & Fluoxetine & $0.099 \pm 0.36$ & $0.31 \pm 0.64$ \\
\hline Paretic & Beta & Placebo & $-0.18 \pm 0.28$ & $0.27 \pm 0.39$ \\
\hline & & Fluoxetine & $-0.022 \pm 0.34$ & $0.40 \pm 0.45$ \\
\hline
\end{tabular}

\section{Execution versus imagination}

The theta, alpha and beta frequency bands measured at C3 and C4, desynchronized significantly more in the contralateral hemisphere during the execution of the movement of the paretic arm $(-0.24 \pm 0.23)$ compared to imagination of the movement of the paretic arm $(-0.073 \pm 0.35)(\mathrm{p}=0.018)$.

\section{Lateralization execution versus imagination}

No significant differences of the lateralization were found between imagination and execution. For both tasks a higher lateralization towards the non-affected hemisphere is found in almost all tested conditions, that is a more negative lateralization during a task with the healthy hand and a more positive lateralization during a task executed with the affected hand (table 2).

\subsection{Discussion}

In this randomized, double-blind, placebo-controlled, crossover trial, we found an increased ipsilateral cortical activity over the affected motor cortex after fluoxetine $20 \mathrm{mg}$ during the execution of a movement of the healthy hand. The significant desynchronization of rhythms in the alpha frequency bands during imagination indicates the possibility of stroke patients to imagine a movement of the affected hand.

Our previous study found an increased muscle activity of both agonistic and antagonistic wrist muscles after intake of a single dose of fluoxetine (20 mg) (Berends, 2009). It can be hypothesized that the increased muscle activity relates to increased activity of the motor areas in the brain. The present study found a desynchronization of the alpha frequency band over the affected motor cortex 
during the movement of the healthy hand. Changes in the power of the alpha frequency band are thought to relate to alertness and expectancy (lower alpha frequency band), and performance of a task (higher alpha frequency band) (Klimesch, 1998). The increased ERDS of the alpha frequency band after intake of fluoxetine might be caused by an increased arousal. In addition, the higher desynchronization of the ipsilateral affected hemisphere might indicate a higher attribution of this hemisphere to the given task (Pfurtscheller, 1999). However, it is surprising that this increased activity was not found in the contralateral hemisphere, or during imagination of the affected arm.

Fluoxetine did not affect the power of the theta and beta frequency bands during movement or imagination of the paretic hand. The beta frequency band is thought to relate to high focus and concentration (Pfurtscheller, 1997). Therefore, fluoxetine did not seem to affect the concentration of the patients. Previous studies found decreased amplitudes of the post-movement beta event related synchronization response after physical therapy (Wilson, 2011). Furthermore, a reduction of movement-related event related desynchronization of the beta frequency band after stroke was found. This reduction of movement-related ERDS was related to the degree of motor impairment (Rossiter, 2014). In another study including the very same patients, we found no influence of a single dose of fluoxetine $(20 \mathrm{mg}$ ) on the motor function and muscle activity (chapter 4). Therefore, it is not surprising that no changes were found in the beta desynchronization.

The limited effects of fluoxetine found in the present study might be caused by several variables. Chronic stroke patients might be less prone to the effects of fluoxetine compared to acute stroke patients. In acute stroke patients, the effects of stroke on processes in the brain might cause fluoxetine to have a larger effect on the excitability compared to the effects in the chronic stage, resulting in increased brain plasticity. Alternatively, it might be that the used dosage of $20 \mathrm{mg}$ fluoxetine was too low to induce an effect during the movement of the paretic hand and during imagination. However, a single intake of $20 \mathrm{mg}$ of fluoxetine is previously found to be sufficient in stroke patients (Dam, 1996; Pariente, 2001; Pleger, 2004; Berends, 2009). Additionally, the present study found a significant desychronization in the (affected) ipsilateral hemisphere after fluoxetine during execution of the healthy hand, indicating an effect of fluoxetine. This result might have been caused by an increased synaptic activity and neurotransmitter synthesis. When the increased activity remains during rehabilitation, it might promote the regeneration of nerves, which is important to be able to relearn movements. 
The attribution of imagination to physical therapy is the ability to train although the muscles are not willing or fatigued by the physical training. The desynchronization of the affected hemisphere is smaller during the imagination compared to the execution of the paretic hand, while the lateralization of the desynchronization during both tasks with the paretic hand is more to the nonaffected hemisphere. This increased ipsilateral activity compared to a contralateral activity was previously found to be related to a reduced recovery of motor function (Ward, 2003; Biernaskie, 2005; Tangwiriyasakul, 2014). In these highly affected patients, the non-affected hemisphere is found to show a higher activation in order to take over the function of the affected site. The chronic stroke patients included in the present study had to have an impaired motor function of the hand, but at the same time be able to generate some muscle activity. Although the functional ability of the patients widely varied, most patients did not show good recovery, confirming the results of previous studies. Although most patients were barely able to execute the movement, desynchronization of the frequency bands during imagination indicates the possibility of stroke patients to imagine the movement.

In conclusion, the desynchronization of EEG rhythms during imagination of the movement shows the possibility of stroke patients to imagine a movement of the paretic hand which cannot be executed. The induced cortical activity might promote cortical plasticity and supports the addition of movement imagination to the established rehabilitation programs.

Fluoxetine did not affect the desynchronization during movement or imagination of a movement of the affected hand. However, the increased ERD over the paretic cortex during movement of the healthy hand after $20 \mathrm{mg}$ of fluoxetine indicates the possibility of fluoxetine to affect cortical activity. This effect of fluoxetine might cause the rehabilitation of motor function after stroke to improve, but optimization of the administration is essential. 


\subsection{References}

Berends HI, Nijlant J, Van Putten MJAM, Movig KLL, IJzerman MJ. Single dose of fluoxetine increases muscle activation in chronic stroke patients. Clin Neuropharmacol 2009 32:1-5

Berends HI, Wolkorte R, Ijzerman MJ, van Putten MJAM. Differential cortical activation during observation and observation-and-imagination. Exp Brain Res. 2013;229:337-345

Biernaskie J, Szymanska A, Windle V, Corbett D 2005. Bihemispheric contribution to functional motor recovery of the affected forelimb following focal ischemic brain injury in rats. Eur J Neurosci 21 989-999

Buccino G, Solodkin A, Small S. Functions of the mirror neuron system: implications for neurorehabilitation. Cog Behav Neurol 2006; 19:55-63.

Calmels C, Holmes P, Jarry G, Lévèque J, Hars M, Stam C. Cortical activity prior to, and during, observation and execution of sequential finger movements. Brain Topogr. 2006; 19:77-8

Chae J, Yang G, Park B, Labatia I. Muscle weakness and co-contraction in upper limb hemiparesis: relationship to motor impairment and physical disability. Neurorehabil Neural Repair 2002; 16: 241-248

Chollet F; Tardy J, Albucher JF, et al. Fluoxetine for motor recovery after acute ischaemic stroke (FLAME): randomised placeb-controlled trial. Lancet Neurol 2011: 10(2): 123-130.

Cirstea MC, Levin MF. Compensatory strategies for reaching in stroke. Brain 2000 123:940953

Dam M, Tonin PDBA, Pizzolato G, et al. Effects of fluoxetine and maprotiline on functional recovery in poststroke hemiplegic patients undergoing rehabilitation therapy. Stroke 1996;27:1211-1214.

Ertelt D, Small S, Solodkin A, Dettmers C, McNamara A, Bikofski F, Buccino G. Action observation has a positive impact on rehabilitation of motor deficits after stroke. Neuroimage 2007; 36:T164-T173

Ilic T, Korchounov A, Ziemann U. Complex modulation of human motor cortex excitability by the specific serotonin re-uptake inhibitor sertraline. Neuroscience Letters 2002;319:116120.

Jacobs B, Fornal C. Activity of serotonergic neurons in behaving animals. Neuropsychopharmacology 1999;21:9S-15S.

Kalcher J, Pfurtscheller G. Discrimination between phase-locked and non-phase-locked event-related EEG activity. Electroencephalogr Clin Neurophysiol 1995;94:381-384 
Klimesch W, Doppelmayr M, Russegger H, Pachinger T, Schwaiger J (1998) Induced alpha band power changes in the human EEG and attention. Neursci Let 244:73-76

Krakauer JW 2006 Motor learning: its relevance to stroke recovery and neurorehabilitation. Curr Opi Neurol 19 84-90

Kwakkel G, Kollen B, Lindeman E 2004 Understanding the pattern of functional recovery after stroke: facts and theories. Restor Neurol Neurosci 22 281-299

Loubinoux I, Boulanouar K, Ranjeva J, Carel C, Berry I, Rascol O. Cerebral functional magnetic resonance imaging activation modulated by a single dose of the monomine neurotransmission enhancers fluoxetine and fenozolone during hand sensorimotor tasks. Journal of Cerebral Blood Flow and Metabolism 1999;19:1365-1375.

Loubinoux I, Pariente J, Boulanouar K, et al. A Single Dose of the Serotonin Neurotransmission Agonist Paroxetine Enhances Motor Output: Double-Blind, PlaceboControlled, fMRI Study in Healthy Subjects. NeuroImage 2002;15:26-36.

Mäkelä JP, Lioumis P, Laaksonen K, Forss N, Tatlisumak T, Kaste M, Mustanoja S. Cortical excitability measured with nTMS and MEG during stroke recovery. Neural Plast. 2015; doic: $10.1155 / 2015 / 309546$

Mead GE, Hsieh CF, Hackett M. Selective serotonin reuptake inhibitors for stroke recovery. JAMA 2013 11;310:1066-1067

Page S, Levine P, Leonard A. Mental practice in chronic stroke. Results of a randomized, placebo-controlled trial. Stroke 2007; 38:1293-1297.

Pariente J, Loubinoux I, Carel C, et al. Fluoxetine modulates motor performance and cerebral activation of patients recovering from stroke . Ann Neurol 2001;50:718-729.

Pfurtscheller G, Neuper C, Andrew C, Edlinger. G Foot and hand area mu rhythms. Int J Psychophysiol 1997 26:121-135

Pfurtscheller G, Lopes da Silva FH. Event-related EEG/MEG synchronisation and desynchronisation: basic principles. Clin Neurophysiol 1999 110:1842-1857

Pleger B, Schwenkreis P, Grünberg C, Malin J-P, Tegenthoff M. Fluoxetine facilitates usedependent excitability of human primary motor cortex. Clinical Neurophysiology 2004;115:2157-2163.

Rossiter HE, Boudrias MH, Ward NS. Do movement-related beat oscillations change after stroke? J Neurophysiol 2014;112:2053-2058 
Solis-Escolante T Müller-Putz GR, Pfurtscheller G, Neuper C. Cue-induced beta rebound during withholding of overt and covert foot movement. Clin Neurophaysiol 2012: 123:11821190

Tangwiriyasakul C, Verhagen R, Rutten WLC, Van Putten MJAM. Temporal evolution of event-related desynchronization in acute stroke: a pilot study. Clinical neurophysiology 2014. 125: 1112-1120

Ward NS, Brown MM, Thompson AJ, Frackowiak RS 2003. Neural correlates of motor recovery after stroke: a longitudinal fMRI study. Brain 126 2476-2496

Wilson TW, Fleischer AN, Archer D, Hayasaka S, Sawaki L. Oscillatory MEG motor activity reflects therapy-related plasticity in stroke patients. Neurorehabil Neural Repair 2011 Feb $13 ; 25: 188-93$

Zittel S, Weiller C, Liepert J. Citalopram improves dexterity in chronic stroke patients. Neurorehabil Neural Repair 2008 22: 311-314 



\section{CHAPTER 7}

General discussion and future perspectives 
In this thesis two interventions are examined which aim to improve the recovery of motor function after stroke. The first was the use of psychoactive drugs, the second was the use of motor imagination. The potential impact of these interventions on cortical activity, muscle activation and motor function was studied.

\subsection{Recovery after stroke}

The ability of the brain to adapt to the new situation after stroke concerns several neuronal mechanisms, which are related to the recovery of motor function. This adaptation can be defined as neuronal plasticity (Chen, 2002). The mechanisms that contribute to the reorganization include recovery of neurons in the penumbra, activation of previously silent synapses and pathways, and take-over of function by the contralesional hemisphere (Chen, 2002; Carmichael, 2003; Rossini, 2004). These processes might be facilitated by drugs like amphetamines, methylphenidate, levodopa and selective serotonin reuptake inhibitors.

The potential effects of these drugs on the recovery of motor function after stroke have been examined. In our review (Chapter 2) 6 randomized controlled trials (RCTs) were described examining the effects of long term use (at least 2 weeks) of various drugs on motor function and motor control. Motor control was defined as the ability to use the muscles in order to accomplish a particular task. Motor function was defined as the ability to function in daily life. None of the included RCTs found an effect on motor control in the stroke population. It was shown that motor function improved in the patients groups treated with methylphenidate, trazodone, and nortriptyline. The results for fluoxetine and levodopa were contradicting and no relationship was found between the affected neurotransmitter and change in motor function. This lead to the assumption that the results of these studies were affected by confounders. Suggested confounders are cognitive impairments or depression, and the interference of the tested medicine on the mental state (chapter 2). Other explanations for the contrasting results are differences between sites of stroke, time after stroke onset, degree of impairment, dose of drugs, and duration of the administration. All of these confounders have to be excluded before final conclusions can be drawn on the influence of psychoactive drugs on the recovery of motor function after stroke.

Based on the results found in the review, the effects of a selective serotonin reuptake inhibitor (SSRI) fluoxetine were further examined in this thesis. Fluoxetine is an SSRI which is widely used as antidepressant in the stroke patient population. It increases the concentration of serotonin in the synaptic cleft between neurons in the brain. Serotonin is assumed to facilitate motor output. It is thought to play an auxiliary role in coordinating appropriate autonomic and neuroendocrine outputs to the ongoing tonic or repetitive motor activity (Jacobs, 1999). After administration of a single dose of SSRI in humans, changes in brain 
activity were found that are hypothesized to facilitate neuroplasticity (Loubinoux ${ }^{\mathrm{a}}$,1999; Ilic, 2002; Loubinoux ${ }^{\mathrm{b}}$, 2002). Motor function after stroke is found to correlate with muscle weakness, co-contraction and abnormal delayed initiation and termination times of muscle contraction (Hammond, 1988; Chae, 2002; Chae 2002). It is therefore hypothesized that when motor function after stroke improves after administration of fluoxetine these muscle activation patterns should change accordingly. To examine this hypothesis, the effects of a single dose of fluoxetine $20 \mathrm{mg}$ on muscle activation patterns and motor function in chronic stroke patients were examined and described in chapter 3 . A significant increase in muscle activity was found in the paretic arm in both the agonist and antagonistic muscle, while no changes in co-contraction were found. No significant effects on delay times and on motor function were found. This non-specific effect is suggested to be caused by the widespread influence of serotonin in the brain and spinal cord.

After fluoxetine, a hyperactivation in the ipsilesional primary motor cortex was found during execution of a movement (Pariente, 2001) as well as the excitability of corticospinal neurons and spinal motorneurons (Ilic, 2002; Pleger, 2004). When the muscle activity increases after administration of fluoxetine (Chapter 3) changes in activity of the motor cortex can be expected. Chapter 4 examined the relation between muscle activation and cerebral activity after intake of a single dose of fluoxetine $20 \mathrm{mg}$ in chronic stroke patients. Although we found a changed muscle activation of the paretic arm in a first experiment, it could not be reproduced in consecutive experiments. Nor were any relations found between cortical activity, muscle activation and motor function. The reasons for these contrasting results between the two studies examining the effects of fluoxetine 20 $\mathrm{mg}$ are uncertain. The in- and exclusion criteria, the methods used and the analyses performed were identical in both experiments. The review of chapter 2 concluded that many confounders have to be excluded in order to better understand the effects of psychoactive drugs on motor function. Confounders mentioned there were differences between sites of stroke, time after stroke onset, degree of impairment, dose of drugs, and duration of administration. Of these confounders, the time after stroke onset, dose of drugs and duration of administration were the same. The site of stroke was defined as "cortical or subcortical infarct". The degree of impairment was defined as "possibility to selectively move paretic thumb and perform dorsal flexion of the wrist". These definitions allow a large group of patients to be included with a variety of kinds of stroke and impairments.

For future studies more strict in- and exclusion criteria might therefore be needed. Unfortunately, by examining the effects in a more restricted patient group, translation of positive findings into clinical administration to a broad patient group will be more problematic. 
The study of Chollet et al (2011) is the only study published today which examined the effects of long-term administration of fluoxetine on motor function shortly after stroke, in a large group of stroke patients $(n=118)$. They found that long-term administration of fluoxetine in subacute ischemic stroke patients has a positive effect on the rehabilitation of motor function, but not on overall impairments caused by stroke including sensory, visual, and cognitive impairments. However, follow-up was only until 95-97 days after stroke, and patients are not yet classified to be "chronic stroke patients". Until that time, the rehabilitation of motor function is not yet stabilized and might be improved by activities in daily living. It cannot be excluded that improvement of motor function was only speeded up by the administration of fluoxetine, without an effect on the final motor function outcome. Therefore, a longer follow-up examining the effects 6 months and 1 year after stroke is warranted. In addition, it has to be studied if the effects of fluoxetine endure after discontinuing the administration. The adaptation of the brain to the increased amount of serotonin might cause a withdrawal effect, and, in the "worstcase scenario", the motor function could even decrease when fluoxetine is discontinued (Berends et al, 2011)

\subsubsection{Imagination of movement}

Neuroplasticity after stroke inducing relearning of motor function only occurs when involved areas are activated. While motor areas in the brain are activated during observation and/or imagination of a movement, it can be hypothesized that next to training by execution of the movement, a training applying observation and/or imagination might also be beneficial for the recovery of motor function. Previous studies examining the effects of action observation (Ertelt, 2007), motor imagery (Schuster, 2012) and mental practice (Page, 2007) support this hypothesis.

Chapter 5 addresses the question which of the different tasks, observation and/or observation-and-imagination, has most clinical relevance in the rehabilitation after stroke. Differences between observation and observation-and-imagination were examined. We found a larger desynchronization over the hand area of the motor cortex in all frequency bands during observation-and-imagination compared to observation-only, during almost the whole activity fragment. The higher alpha frequency band, which is suggested to play a role in the performance of the task, desynchronized more during observation-and-imagination. Observation-andimagination demands more mental focus, which is thought to explain the higher desynchronization of the beta frequency band during this task compared to observation-only. Assuming that a larger desynchronization reflects a larger participation of neurons, these findings suggest an additive effect of imagination to observation. 


\subsubsection{Fluoxetine and imagination of movement}

Both the administration of fluoxetine and the imagination of a movement are found to be potential useful additives to the existing rehabilitation therapies applied to relearn motor function after stroke. These two opportunities to improve the rehabilitation can also be combined and might reinforce each other. Chapter 6 described a randomized double-blind, placebo-controlled, cross-over trial examining the effects of a single dose of fluoxetine $20 \mathrm{mg}$ on cortical activity over the hand area of the motor cortex during execution and imagination of a hand movement. A significant desynchronization over the motor cortex during imagination compared to rest confirms the ability of stroke patients to imagine a motor task they are not able to execute the way it is shown. However, a significantly higher desynchronization was found over the motor cortex during execution compared to observation-and-imagination. After intake of fluoxetine, a significant higher desynchronization was found in the alpha frequency band during execution of the movement. Although not significant, a comparable trend was found during the imagination of a movement. These results indicate a reinforcing effect of fluoxetine on the synaptic activity and neurotransmitter synthesis. When the increased activity remains during rehabilitation, it might promote the regeneration of nerves, which is important in the relearning of movements.

\subsection{On the effects of fluoxetine combined with imagination of a movement}

In chapter 6, the effects of fluoxetine on cortical activity during imagination and execution of a particular hand movement were examined. The significant desynchronization found over the motor cortex during imagination compared to rest confirms the ability of stroke patients to imagine a motor task they are not able to execute the way it is shown. This is in contrast with the results of a study by Orgs et al. (2008), showing that the cortical activity in subjects that were not able to execute the observed movement did not increase. It seems that stroke patients still have the potential or the "cortical abilities" to imagine a movement they were once able to execute. This potential to imagine the movement might be very important when including imagination into a rehabilitation therapy. The intention to include imagination is to "train the brain" and try to create neuronal pathways by inducing neuroplasticity, like it is found during the execution of a movement. Assuming that the degree of desynchronization is important for the neuroplasticity to occur, the execution of a movement cannot be substituted by the imagination, since we found that the cortical activity during execution is higher than it is during imagination. In turn, imagination should be preferred over observation-only (chapter 5). If the physical training of a movement is exhausting for the patient, the training might be pursued by training using imagination.

In chapter 6, fluoxetine $20 \mathrm{mg}$ was found to increase the ipsilateral cortical activity over the affected motor cortex during the execution of a movement. In addition, 
although not statistically significant, the desynchronization during imagination seemed to increase as well. The higher desynchronization of the ipsilateral affected hemisphere might indicate a higher attribution of this hemisphere to the given task, which might facilitate the processes inducing neuroplasticity.

The administration of fluoxetine during movement therapy including execution and imagination of a particular movement seems to be a reinforcing combination in the recovery of motor function after stroke.

\subsection{On the utility of statistics in our research}

The use of statistics to show an effect is amongst the most established ways of analysis in science. Although I support the use of statistics, non-significant results can contain valuable information. Disregarding (trends in) these non-significant data might lure into throwing the baby out with the bath water.

In our studies we used a $p$-value $(p<0.05)$ to define statistically significant results. Another possibility to analyse the data is by calculating the "number needed to treat" (NNT). NNT describes the number of patients that need to be treated to realize one good outcome or to prevent one bad outcome. Then "outcome" has to be defined. For example: " 3 patients out of 10 should show a clinical significant effect on the FM score after 6 months of therapy using imagination of movements". In our research however, examining the effects of a single dose, we were not able to define an effect since no clinical significant effects were to be expected. Therefore it was not possible to use the NNT in this phase of the study. Another possibility would be to examine individual effects or to determine groups of responders and non-responders. However, the within-subject variability was found to be high, making it hard to differentiate between responders and nonresponders. The high within-subject variability of the cortical activity makes us assume that other confounders play a role in the reactivity of the frequency bands. Many stroke patients had difficulty in concentrating during the measurements, some fell asleep and others could not sit still during the EEG-measurements. These cognitive impairments have a large impact on the results. On the other hand, fluoxetine might also affect these cognitive abilities as the increased amounts of serotonin are found in areas of the brain involved in cognition as well. Future research should take all areas that might be involved into account when examining the cortical effects of fluoxetine.

\subsection{On the difference between statistically significant and clinically relevant results}

A single dose of fluoxetine may have only a relatively small effect on cortical activity, muscle activity and motor function. Hence it is important to consider the difference between statistically significant and clinically relevant results. Thus, although the results of chapter 3 and chapter 4 seem to be contradicting, a closer 
look at the trends in the non-significant data provides us with a more detailed view. When the data of chapter 4 are reconsidered, the muscle activation in the paretic arm of the agonist during both extension and flexion is higher after fluoxetine compared to placebo, although not statistically significant. The antagonistic muscle activity was found to be higher after fluoxetine in the first experiment (chapter 3). In our latest experiment (chapter 4), a slightly higher activity was found after fluoxetine during extension of the affected arm, while only a slightly lower activity was found during flexion of the affected arm. Thus, although statistical analysis did not show significant results $(p \leq 0.05)$, these trends largely confirm each other, thereby indicating that a single dose of fluoxetine does increase the muscle activity of agonistic muscles.

An increased muscle activity was found to correlate with cortical activity. A single dose of fluoxetine $20 \mathrm{mg}$ increases muscle activity and might increase cortical activity over the motor cortex in chronic stroke patients. These changes do not affect the motor function after a single dose. This latest result is not surprising. Previous research has shown that only a combination of administration of SSRIs and physical training affects the motor function (Dam, 1996). However, changed muscle activity after intake of fluoxetine indicates a potential effect of fluoxetine on motor function. Muscle weakness is previously found to be related to the degree of impairment (Chae, 2002): when the muscle activity is increased after fluoxetine the muscle weakness is expected to decrease, which would likely improve the motor function. However, this effect might be nullified when the antagonistic muscle activity is increased as well. It might even worsen the motor function when spasticity is increased. The effects of the combination of fluoxetine and physical training should give more insight in these effects of increased muscle activity.

No relations were found between changed muscle activity and changed motor function. Examinations of the cortical desynchronization during execution and also during imagination show a high between and within subject variability (Tangwiriyasakul, 2013). These experiments included healthy subjects. The variability in the stroke population can be expected to be even higher. When examining the differences of an intervention, a paired test can be used analysing the same measures. However, when two different measures are correlated, like cortical activity and muscle activity, this variability might impact the results and it has to be questioned whether these two measures are likely to be correlated at all. For example, when patient 1 shows only a small desynchronization, the small change might be enough to affect muscle activity. Patient 2 might show the same desynchronization which does not influence the muscle activity. In this patient the desynchronization may have to be larger to induce changes in muscle activity. 


\section{Key findings}

The goal of the thesis was to contribute to the understanding of the effects of administration of psychoactive drugs and the imagination of movement on the recovery of motor function after stroke. At the start of this project, the understanding of the psychoactive drugs to relearn motor function after stroke was ambiguous. In addition, the effects of imagination after stroke had to be better comprehended before imagination therapy was ready for considering it in a rehabilitation program.

The results of the examinations described in this thesis are not conclusive about the effects of fluoxetine and imagination. However, there are some lessons learned regarding the use of fluoxetine and imagination in the rehabilitation of motor function after stroke:

\section{Single dose of fluoxetine}

- A single dose of fluoxetine $20 \mathrm{mg}$ does not affect motor function.

- Administration of a single dose of fluoxetine $20 \mathrm{mg}$ does increase agonistic muscle activity.

- Cortical activity of the ipsilateral motor cortex increases after fluoxetine $20 \mathrm{mg}$ during the execution of a movement of the healthy hand.

\section{Imagination}

- Patients that are not able to execute a movement properly still are able to imagine this movement.

- Cortical activity of the motor cortex is higher during execution of a movement compared to observation-and-imagination of that particular movement.

- Cortical activity of the motor cortex is higher during observation-andimagination-only of a movement compared to observation-only of that particular movement.

\section{Imagination and administration of fluoxetine}

- Administration of fluoxetine $20 \mathrm{mg}$ increases cortical activity over the motor cortex during imagination. 


\subsection{Recommendations for future research}

An important question to be answered before fluoxetine can be clinically used to improve motor recovery is whether the results of a single dose of fluoxetine can be extrapolated to effects after long term use. Contradictory to the results found using a single dose of fluoxetine, long term administration of serotonergic drugs was found to decrease the excitability and activation of the primary motor cortex (Gerdelat-mas, 2005; Acler, 2009). During long term administration in stroke patients, postsynaptic serotonin receptors are thought to adapt to the increased amounts of serotonin and become less sensitive, causing a decreased excitability (Acler, 2009) and a hypoactivation (measured in healthy subjects) (Loubinouxc, 2005).

Although it is likely that fluoxetine does have an effect on motor function, the way of action is not unambiguous. It should be carefully monitored what the long term effects are on the adaptation of the serotonergic receptors in the brain and through this on cortical activity, muscle activity and ultimately motor function. Therefore, it would be important to more thoroughly examine long term effects of fluoxetine. Optimally, this should be done in subacute and in chronic stroke patients. During the subacute stage after stroke, motor function is found to have the largest recovery. Therefore, a stronger effect of fluoxetine can be expected shortly after stroke. From a scientific perspective, chronic stroke patients are easier to examine since spontaneous recovery does not occur anymore and there is less or no influence of other therapies affecting the results as the intensity of already applied therapies is decreased or therapies are fully completed. Besides an effect on the final outcome of motor function, the effect of administration of fluoxetine and also of imagination of movement might also lie in the speed of recovery of motor function. That is, the eventual motor function in the chronic stage is the same, but this degree of recovery is reached earlier or later. Therefore, it is advised to persevere with treatment until no further improvement of motor function is experienced.

When examining the effects of long term therapy using imagination of a movement, it is recommended that imagination is added to the existing physical therapy; it should not substitute physical exercise. The cortical activity during execution is higher compared to imagination, and is therefore expected to be more effective in inducing neuroplasticity. However, physical exercise is exhausting the patient. Imagination of movement might be a good option when physical exercise has to be terminated. Like in the research for the effects of fluoxetine, it is important to understand why imagination does, or does not, influence the motor function. The changes of cortical activity over time during the rehabilitation period, in combination with changes in muscle activity and motor function will provide valuable information about the actions of imagination. When adding imagination to an established rehabilitation program, the patients have to show discipline. The 
established rehabilitation therapies are already very exhausting for the patients. To mentally practice a movement which you are not able to perform might be very frustrating and could even interfere with other applied rehabilitation techniques. One should carefully observe the patients in order to exclude potential harmful effects.

Since fluoxetine is found to increase cortical activity, both interventions might reinforce each other. When the actions of both long term use of fluoxetine and imagination are better known the combination might further improve the results of the rehabilitation therapies in stroke. 


\subsection{References}

Acler M, Robol E, Fiaschi A, Manganotti P. A double blind placebo RCT to investigate the effects of serotonergic modulation on brain excitability and motor recovery in stroke patients. J Neurol 2009 : 256 1152-1158

Berends HI, IJzerman MJ, Movig KLL, Van Putten MJAM. Long term administration of fluoxetine to improve motor recovery after stroke. Future Neurology 2011;1

Carmichael ST. Plasticity of cortical projections after stroke. Neuroscientist 2003;9:64-75

Chae J, Yang G, Park B, Labatia I. Muscle weakness and co-contraction in upper limb hemiparesis: relationship to motor impairment and physical disability. Neurorehabil Neural Repair. 2002;16:241-248.

Chae J, Yang G, Park B, Labatia I. Delay in initiation and termination of muscle contraction, motor impairment, and physical disability in upper limb hemiparesis. Muscle \& Nerve. 2002;25:568-575.

Chen R, Cohen LG, Hallett M. Nervous system reorganization following injury. Neuroscience 2002;111:761-773

Dam M, Tonin P, De Boni A, et al. Effects of fluoxetine and maprotiline on functional recovery in poststroke hemiplegic patients undergoing rehabilitation therapy. Stroke. 1996;27:12111214.

Ertelt D, Small S, Solodkin A, Dettmers C, McNamara A, Bikofski F, Buccino G. Action observation has a positive impact on rehabilitation of motor deficits after stroke. Neuroimage 2007; 36:T164-T173.

Gerdelat-Mas A, Loubinoux I, Tombari D, Rascol O, Chollet F, Simonetta-Moreau M. Chronic administration of selective serotonin re-uptake inhibitor (SSRI) paroxetine modulates human motor cortex excitability in healthy subjects 2005: NeuroImage 27, 314-322

Hammond M, Fitts S, Kraft G, Nutter P, Trotter M, Robinson L. Co-contraction in the hemiparetic forearm: quantitative EMG evaluation. Arch Phys Med Rehabil. 1988;69:348351.

Ilic T, Korchounov A, Ziemann U. Complex modulation of human motor cortex excitability by the specific serotonin re-uptake inhibitor sertraline. Neuroscience Letters 2002;319:116120

Jacobs B, Fornal C. Activity of serotonergic neurons in behaving animals. Neuropsychopharmacology 1999;21:9S-15S 
Loubinouxa I, Boulanouar K, Ranjeva J, Carel C, Berry I, Rascol O. Cerebral functional magnetic resonance imaging activation modulated by a single dose of the monoamine neurotransmission enhancers fluoxetine and fenozolone during hand sensorimotor tasks. Journal of Cerebral Blood Flow and Metabolism 1999;19:1365-1375.

Loubinoux $^{b}$ I, Pariente J, Boulanouar K, et al. A Single Dose of the Serotonin Neurotransmission Agonist Paroxetine Enhances Motor Output: Double-Blind, PlaceboControlled, fMRI Study in Healthy Subjects. NeuroImage 2002;15:26-36.

Loubinouxc I, Tombari D, Pariente J et al. Modulation of behaviour and cortical motor activity in healthy subjects by a chronic administration of a serotonin enhancer. NeuroImage 2005: 27 299-313

Miyai I, Reding M. Effects of antidepressants on functional recovery following stroke: a double-blind study. J Neuro Rehab 1998;12:5-13

Orgs G, Dombrowski JH, Heil M, Jansen-Osmann P. Expertise in dance modulates alpha/beta event-related desynchronization during action observation. 2008. Eur J Neurosci 27:33803384 .

Page S, Levine P, Leonard A. Mental practice in chronic stroke. Results of a randomized, placebo-controlled trial. Stroke 2007; 38:1293-1297.

Pariente J, Loubinoux I, Carel C, et al. Fluoxetine modulates motor performance and cerebral activation of patients recovering from stroke. Ann Neurol. 2001;50:718-729.

Pleger B, Schwenkreis P, Grünberg C, Malin J-P, Tegenthoff M. Fluoxetine facilitates usedependent excitability of human primary motor cortex. Clinical Neurophysiology 2004;115:2157-2163.

Rossini PM, Dal Forno G. Integrated technology for evaluation of brain function and neuronal plasticity. Phys Med Rehabil Clin N Am 2004;25:263-306

Schuster C, Butler J, Andrews B, Kischka U, Ettlin T. Comparison of embedded and added motor imagery training in patients after stroke: results of a randomized controlled pilot trial. Trials 2012 13:11

Tangwiriyasakul C, Verhagen R, Van Putten MJAM, Rutten WLC. Importance of baseline in event-related desynchronization during a combination task of motor imagery and motor observation. Journal of Neural Engineering. 201310 p026009 
Summary 
Several studies suggest that plasticity of the brain plays a crucial role in the recovery of motor function after stroke. The main objective of this thesis is to examine the possibility to improve motor function after stroke by modulating brain activity, assuming this would lead to better and long-term motor recovery. Two methods are specifically investigated, i.e. (1) the use of psychoactive agents affecting the concentration of neurotransmitters in the brain, and (2) the use of movement imagination.

When examining the effects of psychoactive agents on motor function after stroke, many confounders like cognitive impairments and depression confuse the outcome. In addition, the locus of the stroke, the degree of impairment, the dose of drugs, and duration of administration are variables that might act on the effects of the psychoactive agents on motor function after stroke. In order to better understand these effects, we decided to examine the impact of fluoxetine on motor function, and relate these outcomes to changes in muscle activity and brain activity. In this way more insight could be obtained in the potential to affect cortical processes improving the recovery of motor function after stroke (Chapter 2).

In the first study examining the effects of administration of a single dose of fluoxetine $20 \mathrm{mg}$ in chronic stroke patients, a significant increase in muscle activity was found in the paretic arm in both the agonistic and antagonistic muscles. No effects were found on motor function. The non-specific effects are suggested to be caused by the widespread influence of serotonin in the brain and the spinal cord (Chapter 3).

The increased muscle activity after intake of fluoxetine is expected to be caused by changes in brain activity over the motor cortex. In our second study examining the effects of fluoxetine, trends were found of an increased agonistic muscle activity and co-contraction in the affected arm after intake of fluoxetine. In addition, a significantly larger brain activity was found in the affected (ipsilateral) hemisphere during execution of the movement by the healthy hand. However, we found no significant relation between the changes in muscle activity and brain activity over the motor cortex. Motor function did not change after intake of fluoxetine (Chapters 4 and 6).

Another intervention possibly facilitating neuroplasticity is to imagine how to execute or to observe a movement. Both of these concepts cause an activation of the motor cortex. When measuring the cortical activity using electroencephalography (EEG), a significantly larger modification of the theta, alpha, and beta frequency bands is found during imagination-and-observation compared to observation-only in healthy subjects. This higher activation of the 
brain persisted during the whole period of imagination, and suggests an additive effect of imagination to observation in the rehabilitation after stroke (Chapter 5).

During execution of a movement, the activity of the motor cortex is significantly higher compared to the activity during imagination of the movement. However, although stroke patients might not be able to actually execute a movement, the motor cortex is found to be activated during imagination. This motor cortex activity suggests that the patients are able to imagine how to execute the movement, despite their non-ability to do it in reality.

Fluoxetine was found to significantly increase the desynchronization of the alpha frequency band in the non-affected motor cortex during the execution of the movement. Trends were found indicating this same effect during the imagination of the hand movement (Chapter 6). Hence the combination of fluoxetine and imagination-and-observation is a promising possibility to improve the rehabilitation of motor function after stroke. 

Samenvatting 
Meerdere studies suggereren dat de plasticiteit van de hersenen een cruciale rol speelt bij het herstel van de motor functie na een cerebraal vasculair accident (CVA). De hoofddoelstelling van dit proefschrift is het bestuderen van de mogelijkheid om het herstel van de motor functie na een CVA te verbeteren door het moduleren van de neurale plasticiteit, waarbij er vanuit wordt gegaan dat dit zal leiden tot een verbeterd herstel op de lange termijn. Twee methodes die hierbij bestudeerd worden zijn (1) het gebruik van psychoactieve medicijnen die de concentratie van neurotransmitters in de hersenen verhogen en (2) het gebruik van inbeelding van een beweging.

Wanneer de effecten van psychoactieve medicatie op de motor functie na een CVA bestudeerd worden, zijn er veel confounders, zoals cognitieve problemen en depressie, die de uitkomsten van de studie beïnvloeden. Andere variabelen die de effecten van de psychoactieve medicijnen op de revalidatie van de motor functie na een CVA kunnen beïnvloeden zijn de locatie van de CVA, de mate van handicap, de dosering van de medicatie en duur van de toediening. Het proefschrift richt zich op het beter begrijpen van de effecten van de selective serotonin reuptake inhibitor fluoxetine. Hiertoe bestuderen we de impact van fluoxetine op de motor functie in relatie tot veranderingen in spieractiviteit en hersenactiviteit. Zodoende willen we meer inzicht krijgen in de mogelijkheid om corticale processen te beïnvloeden die het herstel van de motor functie na een CVA verbeteren (hoofdstuk 2).

In de eerste studie, waarbij het effect van toediening van een enkele dosis fluoxetine $20 \mathrm{mg}$ in chronische CVA patienten werd bestudeerd, is een significante toename van de spieractiviteit gevonden in de aangedane arm in zowel de agonistische als de antagonistische spieren. Een effect op de motor functie werd echter niet gevonden. Er wordt gesuggereerd dat deze a-specifieke effecten veroorzaakt worden door het wijdverspreide effect van serotonine in de hersenen en het ruggenmerg (hoofdstuk 3).

De verhoging van de spieractiviteit na inname van fluoxetine zou veroorzaakt kunnen worden door veranderingen in activiteit van de motorische cortex. In de tweede studie die de effecten van fluoxetine bestudeert, zijn er trends gevonden van een verhoging van de spieractiviteit en de cocontractie van de aangedane arm na inname van fluoxetine. Bovendien werd een significant hogere hersenactiviteit gevonden in de aangedane (ipsilaterale) hemisfeer tijdens het uitvoeren van een beweging met de gezonde hand. Echter, een significante relatie tussen de veranderingen in spieractiviteit en hersenactiviteit van de motorische cortex werd niet gevonden. Motor functie veranderde niet na inname van fluoxetine (hoofdstukken 4 en 6).

Een andere interventie die mogelijk neuroplasticiteit faciliteert is het inbeelden danwel observeren van een beweging. Deze concepten veroorzaken beide een 
activatie van de motorische cortex. Vergeleken met enkel het observeren van een beweging werd, bij gezonde proefpersonen, tijdens het inbeelden van een beweging in combinatie met observatie een significant hogere modificatie van de theta, alfa en beta frequentie banden gemeten met behulp van

elektroencefalografie (EEG). Deze hogere activatie van de hersenen bleef bestaan gedurende de gehele periode van inbeelding en suggereert een hogere activatie van de motorische cortex en een toegevoegd effect van inbeelding van een beweging ten opzichte van enkel observeren voor het herstel van motor functie tijdens de revalidatie na een CVA (hoofdstuk 5).

Op het moment dat CVA-patienten een specifieke beweging trachten uit te voeren is de activiteit in de motorische cortex significant hoger dan tijdens het inbeelden van de beweging. Aangezien de motorische cortex wordt geactiveerd tijdens het inbeelden van beweging wordt verondersteld dat patienten wel degelijk in staat zijn bewegingen in te beelden, ondanks het feit dat zij niet in staat zijn dezelfde beweging uit te voeren. Tijdens de uitvoering van een beweging werd, na inname van fluoxetine, een significante desynchronisatie van de alfa frequentie band in de niet-aangedane hemisfeer gevonden. Tijdens het inbeelden van een beweging zijn trends gevonden voor dezelfde effecten (hoofdstuk 6). Derhalve is de combinatie van fluoxetine en inbeelden-en-observeren van een beweging een veelbelovende mogelijkheid om de revalidatie van de motor functie na een CVA te verbeteren. 

Dankwoord 
Eindelijk is het dan zover: het boekje is klaar! $\mathrm{Nu}$ dan ook eindelijk een goede gelegenheid om mensen te bedanken voor hun hulp.

Allereerst wil ik mijn eerste promotor prof. dr. ir. M.J.A.M. van Putten bedanken. Michel, bedankt dat jij mijn eerste promotor wil zijn. Ik heb erg veel van je geleerd. $\mathrm{Na}$ onze gesprekken ging ik altijd weer met nieuwe kennis en ideeën, maar met minstens zoveel nieuwe vragen de deur uit. Jouw opbouwende manier van terugkoppelen en je kritische blik hebben ervoor gezorgd dat dit proefschrift hier nu ligt. Bedankt voor alles!

Vervolgens wil ik mijn tweede promotor prof. dr. M.J. IJzerman bedanken. Maarten, vanaf mijn afstudeerproject heb je vertrouwen in mij gehad. We hebben, samen met Michel, Juliette en Michiel een mooi onderzoek op kunnen zetten. In het laatste deel van het traject hebben we minder contact gehad, maar ondanks dat ben je je blijven inzetten met dit boekje als resultaat. Bedankt voor je geduld en je vertrouwen!

Ook wil ik graag mijn co-promotor dr. K.L.L. Movig bedanken. Kris, vanaf het begin van mijn promotie ben je betrokken geweest en heb je je ingezet om de studies met fluoxetine mogelijk te maken. Zowel met je inhoudelijke kennis, als met de kennis over de logistieke zaken rond bijvoorbeeld de METC en de procedures rond farmacologisch onderzoek heb je me erg geholpen om de studies op te zetten en uit te voeren. Bedankt!

Tevens een woord van dank aan mijn overige commissie leden prof. dr. Kwakkel, prof. dr. Van Roon, prof. dr. ir. Hermens, prof. dr. Rietman en dr. Hofmeijer. Bedankt voor het nemen van de tijd en moeite om dit proefschrift te lezen en te beoordelen.

Marit, wat ontzettend fijn dat je mijn paranimf wil zijn! We hebben in onze tijd bij RRD als kamergenoten veel samen meegemaakt. Jij was altijd degene die als eerste kon "meegenieten" van m'n frustraties en stress. Maar gelukkig konden we ook de successen, privé-gebeurtenissen en vooral ook koekjes, koppen thee en koffie samen delen. Dank je wel.

Thijs, je hebt me erg kunnen helpen bij vooral het technische gedeelte van het proefschrift. Als ik er even niet uit kwam, nam je altijd de tijd om alles helder en geduldig uit te leggen. Soms zelfs met een lekker etentje vooraf. Ook voor jou geldt dat ik het heel erg fijn vind dat je mijn paranimf wilt zijn!

Er zijn nog veel andere ex-collega's bij RRD die ervoor gezorgd hebben dat ik met een goed gevoel terugkijk op die tijd. Juliette, je bent waarschijnlijk verrast dat het boekje alsnog is gekomen. Ik wil je hierbij heel erg bedanken voor de ontzettend fijne samenwerking die we hadden. Ik heb veel geleerd van jouw klinische blik. Michiel, bedankt voor je begeleiding bij RRD. Vooral aan het ontrafelen van de "spaghetti" die nog wel eens als beeldspraak werd gebruikt voor mijn stukken 
denk ik nog met regelmaat terug. Leendert, ontzettend bedankt voor alle hulp bij de metingen en de analyse. Karin, bedankt voor je adviesen over de statistiek. Daarnaast natuurlijk dank aan alle mede "junior onderzoekers" en andere collega's voor alle gezelligheid, leuke wandelingen en rolstoelbasketbal-toernooien.

Ook de collega's van de UT wil ik bedanken. Ondanks dat ik weinig op de UT aanwezig was, toonden jullie altijd interesse en kon ik jullie altijd benaderen als ik met vragen zat.

Behalve in Enschede heb ik ook in Nijmegen, bij de Sint Maartenskliniek een aantal bijzondere mensen leren kennen. Louis, bedankt voor het blijven aanmoedigen om mijn promotie af te maken. Jouw enthousiasme voor de wetenschap en kritische blik bij alles wat je ziet (of meet) werkt aanstekelijk. Van jou heb ik geleerd om de schakel te maken tussen wetenschap en praktijk en andersom.

Ilona en Mirjam, bedankt voor alle fijne gesprekken die we regelmatig voeren. Het is heerlijk om jullie als luisterend oor te hebben. Zowel de inhoudelijke als de persoonlijke gesprekken houden me scherp. Laten we ons vooral blijven inzetten om deze goede samenwerking en kritische houding te behouden!

Alida, Barend, Agnita, en Marinus dankzij jullie vernieuwende ideeën hebben we, in de korte tijd dat we samenwerken in het $\mathrm{VU}$ medisch centrum, al veel mogelijkheden gecreëerd voor nieuw onderzoek. Hopelijk kunnen we hier in de toekomst een mooie uitwerking aan geven.

Als laatste het stuk wat misschien wel het moeilijkste te schrijven is van het hele proefschrift, het dankwoord aan de familie.

Joke en Carel, bedankt voor alles wat jullie gedaan hebben om mij de kans te geven aan dit proefschrift te werken. Ik ben jullie hier erg dankbaar voor.

Mir, Rich, Miek en Mike, jullie zijn geweldige zussen en schoonbroers! Bedankt voor de heerlijke ontspannen gesprekken, relativerende en motiverende woorden. Lieve pap en mam, waar zou ik geweest zijn zonder jullie steun. Ik kan me er niets bij voorstellen, behalve dat dit proefschrift er niet was geweest. Jullie hebben me altijd en onvoorwaardelijk geholpen. Als ik problemen had of de motivatie niet meer kon opbrengen, wisten jullie me altijd weer op te beuren en op de goede weg te helpen. Dikke kus!

Lieve San, van jou mag ik geen cliché's gebruiken in mijn dankwoord; ik doe m'n best. Het afronden van dit proefschrift heeft op sommige momenten behoorlijk druk gezet op de tijd die we ook samen hadden kunnen doorbrengen; bedankt voor alle ruimte die je me hebt gegeven. En nog veel meer...voor alles: bedankt!

Lieve Fenne en Rens, ook jullie hebben mij erg geholpen. Door gewoon lief te zijn en af en toe een kus of knuffel te komen brengen op zolder. Het is klaar, jullie hoeven nu niet meer te vragen "of ik weer naar zolder moet". Misschien nog 1 keer, om op te ruimen. 



\section{Biografie}


Hanneke Irene Berends - van Genderen werd geboren op 16 januari 1980 in Enschede. In 2001 is ze gestart met de studie Bewegingswetenschappen aan de RijksUniversiteit te Groningen. In 2005 heeft ze deze studie afgerond. Aansluitend is ze begonnen als junior onderzoeker met haar promotieonderzoek bij Roessingh Research and Development. Het onderzoek had als doel het effect van fluoxetine op het motorisch herstel na CVA te bestuderen. Daarnaast richtte het onderzoek zich op de mogelijkheid om het motorisch herstel te faciliteren door middel van het inbeelden van beweging.

Momenteel is ze werkzaam in de Sint Maartenskliniek in Nijmegen. Hier heeft ze een interne opleiding gevolgd voor het intraoperatief monitoren van de neurologische functies (intra-operatieve neuromonitoring: IONM) bij risicovolle orthopedische operaties aan de wervelkolom. In april 2010 is hiervoor het certificaat behaald. De functie wordt gecombineerd met het uitvoeren van wetenschappelijk onderzoek naar het optimaliseren van de IONM.

Daarnaast is ze vanaf 2015 werkzaam in het VU medisch centrum. Ook hier voert ze de IONM uit, in combinatie met wetenschappelijk onderzoek. 


\section{Journal publications}

Multimodality intraoperative neuromonitoring in extreme lateral interbody fusion. Transcranial electrical stimulation as indispensable rearview. HI Berends, HL Journée, I Rácz, J van Loon, R Härtl, M Spruit European Spine Journal 2015 Aug 27

Differential cortical activation during observation and observation-andimagination.

HI Berends, R Wolkorte, MJ Ijzerman, MJAM van Putten

Experimental Brain Research 2013 Sep;229(3):337-45

Long-term administration of fluoxetine to improve motor recovery after stroke HI Berends, MJ IJzerman, KLL Movig, MJAM van Putten

Future Neurology 01/2011

The clinical use of drugs influencing neurotransmitters in the brain to promote motor recovery after stroke; a Cochrane systematic review.

HI Berends, JMM Nijlant, KLL Movig, MJAM van Putten, MJA Jannink, MJ IJzerman European journal of physical and rehabilitation medicine 2009 Dec;45(4):621-30

Single dose of fluoxetine increases muscle activation in chronic stroke patients. HI Berends, JMM Nijlant, MJAM van Putten, KLL Movig, MJ IJzerman Clinical Neuropharmacology 2009 Jan-Feb;32(1):1-5

\section{Conference contributions}

Operative correction of AIS and neuromuscular scoliosis under halo-femoral traction and IONM.

A Stadhouder, HI Berends

Annual meeting of the Nordic Spinal Deformities Society 27-29 August 2015

Neuromonitoring bij XLIF; Corticale stimulatie als dodehoekspiegel HI Berends - van Genderen, HL Journée, I Rácz, K Vullers, M Spruit, J van Loon Spinesymposium Wervelkolomdeformiteit bij volwassenen; Chirurgische strategie en risicomanagement; 19 juni 2014 
Transcranial voltage and current thresholds of muscle potentials in upper and lower extremities related to head impedance and electrode montage

H Berends, HL Journée

4th International Conference on Intraoperative Neurophysiology

ISIN November 14-16 2013, Cape Town

Abstract in: Clinical Neurophysiology. 05/2014; 125(5):e15

Evoked movements in transcranial electrical stimulation on 4 locations of the body at different stimulation electrode montages and stimulation paradigms

E Hoebink, HL Journée, I Rácz, H Berends, C van Hal

4th International Conference on Intraoperative Neurophysiology

ISIN November 14-16 2013, Cape Town

Abstract in: Clinical Neurophysiology. 05/2014; 125(5):e16

Differential cortical activation during observation and imaging

HI Berends, R Wolkorte, T Krabben, MJA Jannink, MJ IJzerman, MJAM van Putten

14th European Congress of Clinical Neurophysiology

21 June 2011 - 25 June 2011

Abstract in: Clinical Neurophysiology 01/2011; 122, Supplement 1:S64 - S65

Intraoperative neuromonitoring during corrective spine surgery using traction HI Berends, AA Gouw, A Stadhouder, HL Journée

5th Congress of International Society of Intraoperative Neurophysiology November 12-14, 2015

Differences between transcranial stimulation electrodes for motor MEP monitoring in corrective spinal cord surgery

HI Berends, A Stadhouder, HL Journée

5th Congress of International Society of Intraoperative Neurophysiology November 12-14, 2015

Influence of a single dose of fluoxetine on brain activity during movement observation, muscle activity and motor function in chronic stroke patients HI Berends, T Krabben, MJ IJzerman, MJAM van Putten

30th International Congress of Clinical Neurophysiology (ICCN) of the IFCN

March 19-23, 2014 
Optimization of the positioning of stimulation electrodes during IONM in scoliosis surgery

HI Berends, HL Journée

$3^{\text {rd }}$ Congress of International Society of Intraoperative Neurophysiology

15-17 September 2011

Brain activity during imaging in stroke patients

HI Berends, MJA Jannink, MJ IJzerman, MJAM van Putten

14th European Congress of Clinical Neurophysiology

21 June 2011 - 25 June 2011

Abstract in: Clinical Neurophysiology 01/2011; 122 


